\title{
Additive Manufacturing of Metamaterials: A Review
}

Meisam Askari ${ }^{1}$, David A. Hutchins ${ }^{2}$, Peter J. Thomas ${ }^{2}$, Lorenzo Astolfi ${ }^{2}$, Richard L. Watson ${ }^{2}$, Meisam Abdi ${ }^{3}$, Marco Ricci ${ }^{4}$, Stefano Laureti ${ }^{4}$, Luzhen Nie ${ }^{5}$, Steven Freear $^{5}$, Ricky Wildman ${ }^{1}$, Christopher Tuck ${ }^{1}$, Matt Clarke ${ }^{1}$, Emma Woods ${ }^{1}$, Adam T. Clare ${ }^{*}$

${ }^{1}$ Department of Mechanical, Material and Manufacturing Engineering, University of Nottingham, University Park, Nottingham, NG7 2RD, UK

${ }^{2}$ School of Engineering, University of Warwick, Coventry CV4 7AL, UK

${ }^{3}$ School of Engineering and Sustainable Development, De Montfort University, The Gateway, Leicester, LE1 9BH, UK

${ }^{4}$ Department of Informatics, Modelling, Electronics and Systems Engineering, University of Calabria, Via Pietro Bucci, 87036 Arcavacata, Rende (CS), Italy

${ }^{5}$ School of Electronic and Electrical Engineering, University of Leeds, Leeds, LS2 9JT, UK

Keywords: metamaterials, additive manufacturing, acoustic, optical, photonic crystals auxetic, phononic

\section{List of Contents}

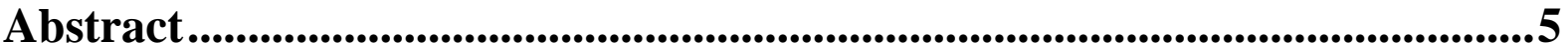

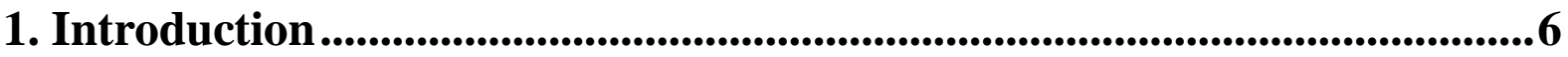

2 Electromagnetic (EM) Metamaterials ......................................................................8

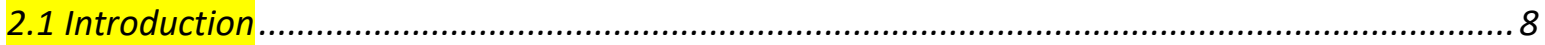

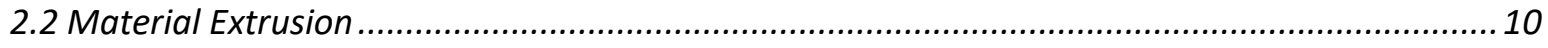

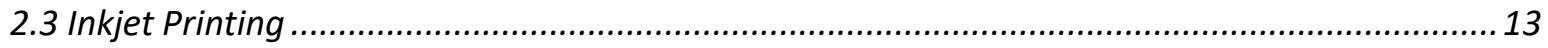

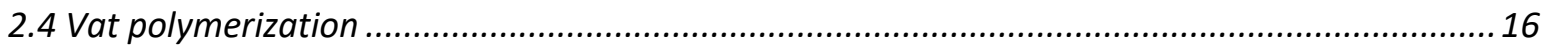

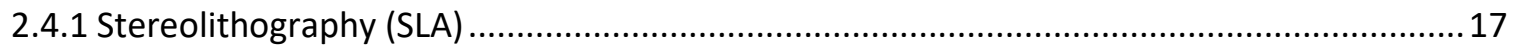

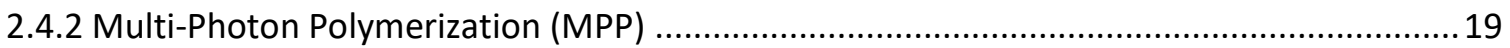

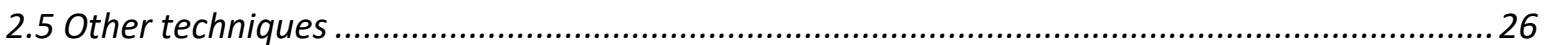

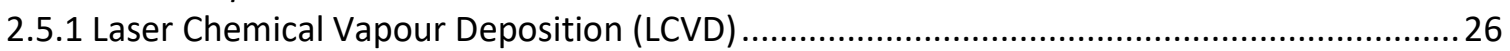

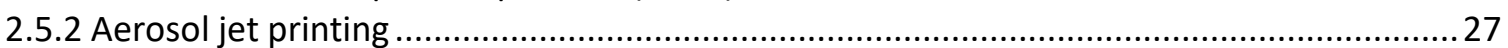

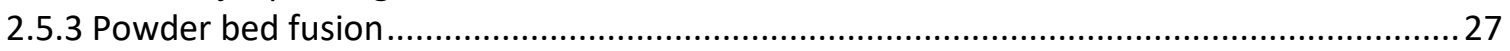

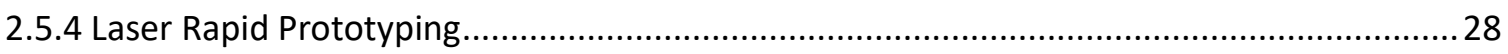

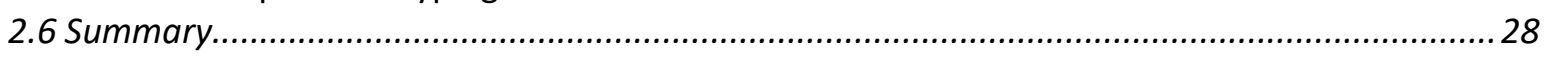

3 Acoustic Metamaterials (AMMs) ...................................................................29

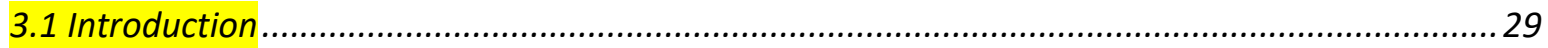

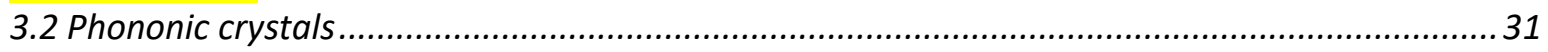

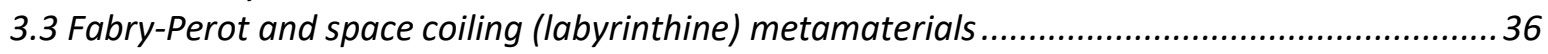

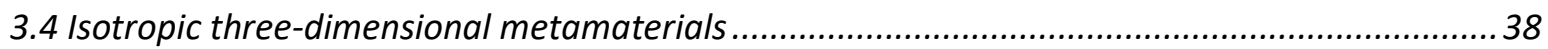

4 Mechanical Metamaterials .......................................................................40

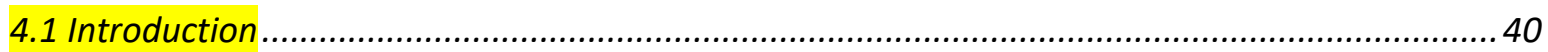

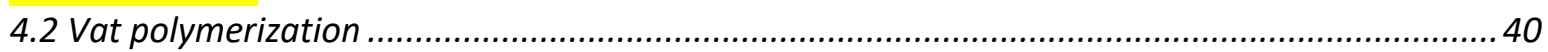


4.3 SLM .

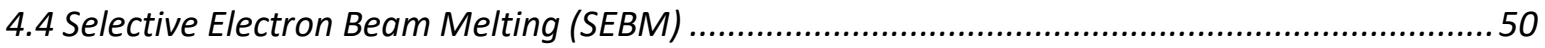

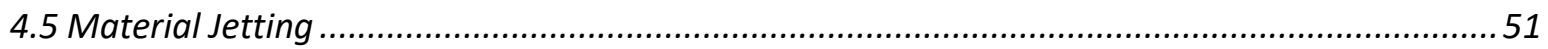

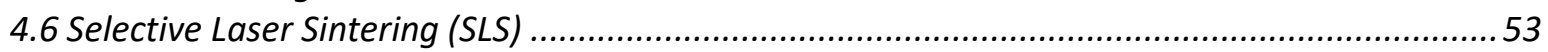

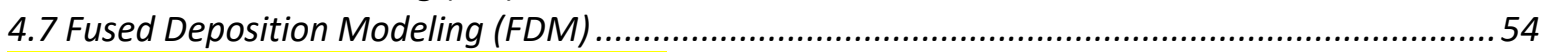

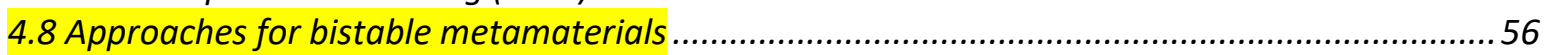

5 Multiple Material Approaches.........................................................................57

6 Conclusions and Directions for Future Research ..............................................60

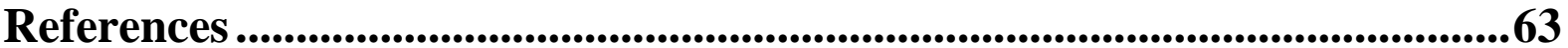

\section{List of Abbreviations:}

AJP: Aerosol Jet Printing

ALD: Atomic Layer Deposition

AMM: Acoustic Metamaterials

AM: Additive Manufacturing

BCC: Body Centre Cubic

CAD: Computer Aided Design

DLW: Direct Laser Writing

DOE: Diffractive Optical Element

EBL: Electron beam lithography

EM: Electromagnetic

FDM: Fused Deposition Modelling

FE: Finite Element

FEM: Finite Element Modelling

FIB: Focused Ion Beam

FTIR: Fourier Transform Infrared

GRIN: Graded Index

LCVD: Liquid Chemical Vapour Deposition

LIT: Laser Induced Transfer

LRP: Laser Rapid Prototyping

MM: Mechanical Metamaterials

MPP: Multi Photon Polymerization

MSL: Micro Stereolithography

NA: Numerical aperture

NTE: Negative Thermal Expansion

PBG: Photonic Bandgap

PC: Photonic Crystals

PCB: Printed Circuit Board

PDMS: Polydimethylsiloxane

PEGDA: Poly(ethylene glycol) Diacrylate

PI: Photo Initiator

PLA: Polylactic Acid

PMMA: Poly methyl methacrylate 
PP: Polypropylene

PnC: Phononic Crystal

2PP: 2 Photon Polymerization

RF: Radio Frequency

SC: Simple Cubic

SEBM: Selective Electron Beam Melting

SEM: Scanning Electron Microscopy

SIMP: Solid Isotropic Material with Penalization

SIJ: Superfine Ink Jet

SLA: Stereolithography

SLB: Selective Laser Burnout

SLM: Selective Laser Melting

SLS: Selective Laser Sintering

SRR: Split Ring Resonator

SRCR: Split Ring Cross Resonators

SPPW: Self-Propagating Photopolymer Waveguide

TEM: Tunnelling Electron Microscopy

TPMS: Triply-Periodic Minimal Surface

\section{List of Figures}

Figure 1: Graph of the number of peer-reviewed articles published with the keyword metamaterials by year [26].

Figure 2: Schematic diagram of the SRR meta-atoms in comparison to the active wavelength of the metamaterial (left) and the energy flow diagram for negative refractive index material (right) [27]

Figure 3: SEM image of a PC fabricated using FDM techniques with a unit cell of $2 \mathrm{~mm}$ and a rod diameter of $0.4 \mathrm{~mm}$ (left). Comparison of the measured and calculated bandgap for this PC structure [60].

Figure 4: SEM photograph of a 3D extruded woodpile structure fabricated from a ceramic slurry. (a) top view of one rod, (b) microstructure and (c) cross-section of the structure, and (d) a cross-section of one rod [72].

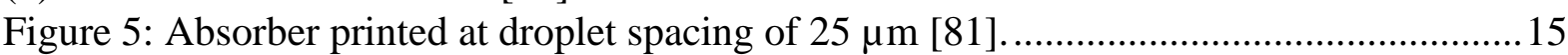

Figure 6: Metamaterial-based ethanol sensor fabricated using inkjet printing [83] .............. 15

Figure 7: Steps in the two-photon polymerization process [98] ........................................ 17

Figure 8 SEM image of chiral PC structure with a layer thickness of $1.32 \mu \mathrm{m}$, a rod diameter of $300 \mathrm{~nm}$ and a lattice constant of $1.2 \mu \mathrm{m}$. The fabricated structure is made of 24 layers with an open window of $60 \mu \mathrm{m}$. The thick wall around the structure decreases sample bending and deformation due to stresses and shrinkage during the polymerization and development process [115].

Figure 9: SEM photographs of a bi-chiral photonic crystal, showing a) top view of different arrangements for photonic crystals, and b) $60 \mu \mathrm{m}$ by $60 \mu \mathrm{m}$ right/left photonic crystal fabricated with a lattice constant of $4 \mu \mathrm{m}$ using MPP [114].

Figure 10: Diffraction gratings fabricated using $5 \mathrm{wt} \%$ gold precursor-loaded polymer. The diffraction pattern of $712 \mathrm{~nm}$ red laser was generated by structures with periodicities of (a) $1.5 \mu \mathrm{m}$, (b) $2.5 \mu \mathrm{m}$ and (c) $5 \mu \mathrm{m}$ [142] 
Figure 11: a) SEM image of a MPP- fabricated bi-chiral photonic crystal, together with b) the process diagram for fabrication, plating and redisposition of the structure on a new substrate [149].

Figure 12: (a) SRR structure fabricated using SU-8 on a silicon substrate. The structure was treated with RF plasma prior to silver coating so that the high-quality metal coating was only applied to the SU-8 structures. (b) Transmission spectra for electric field parallel and perpendicular to the SRR gap show an LC resonance dip at $0.64 \mathrm{THz}$ [151].

Figure 13: Metallic helices fabricated using a combination of stimulated emission depletion assisted MPP and electrochemical metal deposition. (a) to (c) diagram illustrates different steps of fabrication; (d) SEM picture of polymer shells; (e) and (f) show arrays of metallic helices fabricated using negative photoresist, and (g) shows an array of metallic helices fabricated using positive photoresist [154].

Figure 14 Graph of different structures fabricated using AM techniques with their feature sizes and their operating frequency. It is clear that the operation wavelength of the structures is usually smaller than their feature sizes.

Figure 15 2D COMSOL simulation of the irreducible Brillouin zone of a periodic polymer structure with circular holes. The unit cell size of $1 \mathrm{~mm}$ and the hole dimension of $0.475 \mathrm{~mm}$ were chosen in this simulation. a) Plot showing the existence of bandgaps, b) the unit cell and c) irreducible Brillouin zone with marked directions.

Figure 16: (a) Metamaterial structure built by Matlack et al. [188] using FDM, with metal cubes (shown in blue) inserted manually within the polymer grid. (b) The unit cell used in defining the grid structure.

Figure 17: (a) Nylon phononic crystal metamaterial manufactured using SLS [190]............33

Figure 18: (a) Phononic crystal metamaterial structure printed using MSL by Lucklum and Vellekoop [192]. (b) The unit cell used in defining the structure.

Figure 19: Geometries and FEM meshes used by Kruisová et al. [197] for calculations of the band structure of their scaffold structures: (a) SS structure; (b) LS structure; (c) SH structure; (d) LH structure. Schematic sketches on the left show the placements of the unit cells in tetragonal $(\mathrm{a}, \mathrm{b})$ and hexagonal $(\mathrm{c}, \mathrm{d})$ structures in the $\mathrm{x}_{1}-\mathrm{x}_{2}$ plane.

Figure 20: The four unit-cells used by Wormser et al. [201] to fabricate a PnC via EBM having a range of bandgap properties.

Figure 21: The Fabry-Perot metamaterial used in the air by Laureti et al. with $\mathrm{h}=14.8 \mathrm{~mm}$ and $\Lambda=2.96 \mathrm{~mm}[195]$

Figure 22: Resonant structure using concentric cylinders of different lengths for sound absorption in the air [209].

Figure 23: (a) Design of a Fresnel lens using space-coiling techniques, and (b) the lens manufactured using Fused Filament Deposition AM (adapted from [21]).

Figure 24: Spherical acoustic metamaterial structures using (a) space coiling (taken from [20]) and (b) a metamaterial containing a cubic structure (taken from[225]).

Figure 25 Unfeelability cloak fabricated to elastically hide the solid cylinder placed underneath the structure [246].

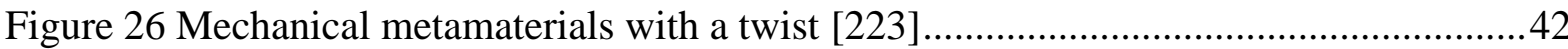

Figure 27 Mechanical metamaterials with tunable negative thermal expansion fabricated using multi-material projection MSL [250].

Figure 29: Glassy carbon nanolattices created by pyrolysis of 3D-printed polymeric microlattices [255]....

Figure 30: Compression experiments on thin-walled and thick-walled ceramic nanolattices. (A to $\mathrm{E}$ ) - mechanical data, compression and recovery of a thin-walled nanolattice. ( $\mathrm{F}$ to $\mathrm{J}$ ) mechanical data, compression and brittle failure of a thick-walled nanolattice. [262] 
Figure 31: (A) Diagram of the SLA system used for fabrication of the lattice structure. (B) to (E) Octet-truss micro-lattice structures fabricated using SLA with different structures. $(\mathrm{F})$ to

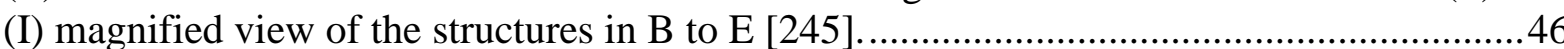
Figure 32: Nanolattice design and hierarchy. (a) A 3D design of a single unit cell of the structure. (b) The 3D design of the full nano lattice structure. (c) SEM image of a section of the fabricated structure. The top-left inset shows the dark field TEM image of the structure which shows the nano-sized grains and the bottom left inset shows TiN hollow tubes [266].47 Figure 33 Comparison of compression behaviour of hollow ceramic (A-C), ceramic-polymer composite (D-F) and solid polymer (G-I) second-order half-cell hierarchical nanolattices

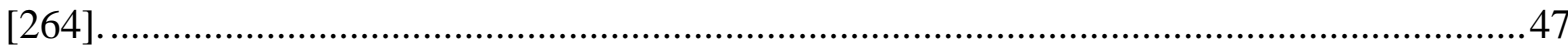
Figure 34 Illustration of fabrication process (b) and breakdown of a structural hierarchy (c-j)

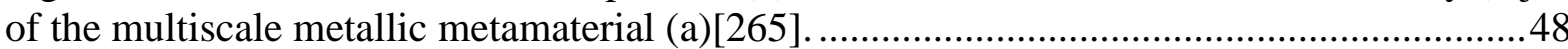
Figure 35 Highly-stretchable mechanical metamaterials made from elastomer [267]..........49 Figure 36 Pentamode Ti-6Al-4V lattice structure made with SLM [238] ............................50 Figure 37 (a) Two halves of the titanium collapsible mould (b) all the components of the mould. The ring, funnel and the internal rod was built of stainless steel. [232] .....................51 Figure 38: Auxetic metamaterial structure fabricated using material jetting (a) top view (b) side view (c) diagram of proposed construct (d) top view and side view of fabricated structure

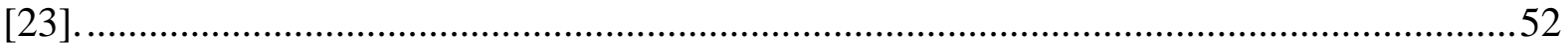
Figure 39: Single actuator soft robot based on one auxetic metamaterial hand [22]..............53 Figure 40 (a) Polyamide structure fabricated using SLS with Poisson's ratio of -0.5 [230] (b) soft auxetic lattice structures made from duel-segment TPU powders [275] (c) Macroscopic polymer auxetic structures with Poisson's ratio of -.08 [233] ..............................................54 Figure 41 FDM printed metamaterial structures for mechanical testing. (a,b,e,f) used in axial tests and (c,d,g,h) used for transverse directions. (a,c,e,g) was manufactured for tension test and $(\mathrm{b}, \mathrm{df}, \mathrm{h})$ was used in compression testing. Dimensions of samples a-h were $132 \times 86.5 \times 3$, $66 \times 86.5 \times 10,142.2 \times 82.5 \times 3,56.8 \times 82.5 \times 10,228.6 \times 132 \times 3,85.7 \times 132 \times 10,231 \times 143.6 \times 3$ and $82.5 \times 143.6 \times 10 \mathrm{~mm}$, respectively [276]

Figure 42 A door latch as a metamaterial mechanism [277]...........................................55

Figure 43 (a) Meta-sandwich structures printed through FDM (b) deformation of metasandwich structures [278].

\begin{abstract}
Metamaterials exhibit properties beyond those exhibited by conventional materials in conventional scenarios. These have been investigated both theoretically and experimentally at length. In many cases the underpinning physical understanding of metamaterials has greatly preceded our ability to manufacture constituent structures. However, the development of additive manufacturing techniques gives new possibilities for the fabrication of complex metamaterial structures, many of which cannot be realised through conventional fabrication methods. The literature to date contains contributions from a diverse group of researchers from the physical sciences, mathematics, and manufacturing technology in the creation of metamaterials for electromagnetic, acoustic and mechanical applications. It is proposed that additive manufacturing holds the key to realise the capabilities of this vibrant research community and permit the creation of new paradigms in fundamental structures but also exploitation through application. For this purpose, a literature review is presented which identifies key advances in metamaterials alongside additive manufacturing and proposes new opportunities for researchers to work together through intra/inter disciplinary research to realise
\end{abstract}


structures which exhibit extraordinary behaviour(s). This review represents a comprehensive account of the state-of-the-art in the production of such metamaterials using additive manufacturing methods and highlights areas, which, based on trends observed in the literature, are worthy of further research and require a coordinated effort on behalf of the afore mentioned disciplines in order to advance the state-of-the-art.

\section{Introduction}

The prefix "meta" in Greek means "beyond"; thus, metamaterials are materials designed to have novel properties that are beyond the normal interactions in the mechanical, acoustic and optical domains. The term 'metamaterial' is defined by the European Union's Metamorphose network as "an arrangement of artificial structural elements, designed to achieve advantageous and unusual electromagnetic properties" [1]. In this definition, it is inherent that metamaterials are manufactured so as to have uncommon properties. Nature already provides some examples of unusual properties. One primtive example is cork, with a Poisson's ratio of almost zero, which also shows little lateral expansion under pressure.

The building block (or the functional unit cell) of the metamaterial is termed the 'meta-atom'. Typically the meta-atom dimensions must be at or lower than the wavelength of the incident wave [1]. Therefore, the inhomogeneous microstructure when aggregated over sufficient length scale is effectively a uniform material from the perspective of the energy source [2,3]. The novel properties of metamaterials are dictated by the structure of the metamaterial, which will be different from its constituent material properties [4]. Effectively, material properties such as permeability, permittivity, refractive index and elastic modulus will be based on the geometry of the structures and the constituent meta-atoms [5]. Therefore, the choice of fabrication method is a critical choice that dictates the resolution, material and working frequency of the resultant metamaterial.

Perhaps the oldest metamaterial structure known is the Lycurgus Cup. Manufactured in the $4^{\text {th }}$ century $\mathrm{AD}$, this ancient cup contained gold and silver particles embedded within a glass matrix. As a result, the transmission spectrum of the material is modified based on the ambient lighting [1]. More recently, realizing metamaterial structures has been conducted by simple assembly methodologies which made use of stacking and arranging layers [6-8] or by applying simple subtractive operations such as drilling into a block of dielectric material [9]. These processes were time-consuming and suffered from inconsistency [5] but yet served to demonstrate the principle and accelerated interest in an emergent topic. The complexity of design is somewhat limited by these methods and hence a superior route to realise these structures across length scales is required.

Alongside the proliferation of advanced lithographic processes, the emergence of low cost and high-resolution laser processing, has allowed metamaterials to be explored more rapidly and for designs to be greatly more complex. In the optical domain, plasmonic absorbers [10], photonic crystals (PC) [11-13], negative index lenses [14] and metamaterial antennas $[3,15,16]$ 
have all been simulated and experimentally tested. The analogies that exist between acoustic waves and electromagnetic waves [17] led to the subsequent study of Phononic Crystals (PnCs) $[18,19]$ and acoustic lenses [20,21]. Mechanical metamaterials with negative Poisson's ratio, negative elasticity and bulk modulus are also another class of metamaterials that greatly benefit from the emergence of advanced manufacturing methods [22,23].

Due to the advancements in nanofabrication and imaging technologies, metamaterial structures have been subject to intense research [24]. The growth in the number of papers published in the metamaterial field from 2000 to 2019 (see Figure 1) clearly demonstrates the rise in metamaterial research. A wide variety of applications is available due to the unique properties of metamaterials. The development of additive manufacturing (AM) has transformed the toolbox available to metamaterial researchers who can now exert control over multiple length scales within single-step processes and exploit entirely new geometrical and material design freedoms. The route to functional metamaterial structures is aided by exploiting wellestablished links between the outputs of numerical modelling systems and computer-aided design. It should be mentioned that, due to the current lack of manufacturing processes able to fabricate large scale metamaterials with complex geometries, most metamaterial applications are yet to be exploited [25]; however, advances in AM are expected to lead to much wider adoption of metamaterials in everyday life in the future. This in turn gives rise to the present review paper.

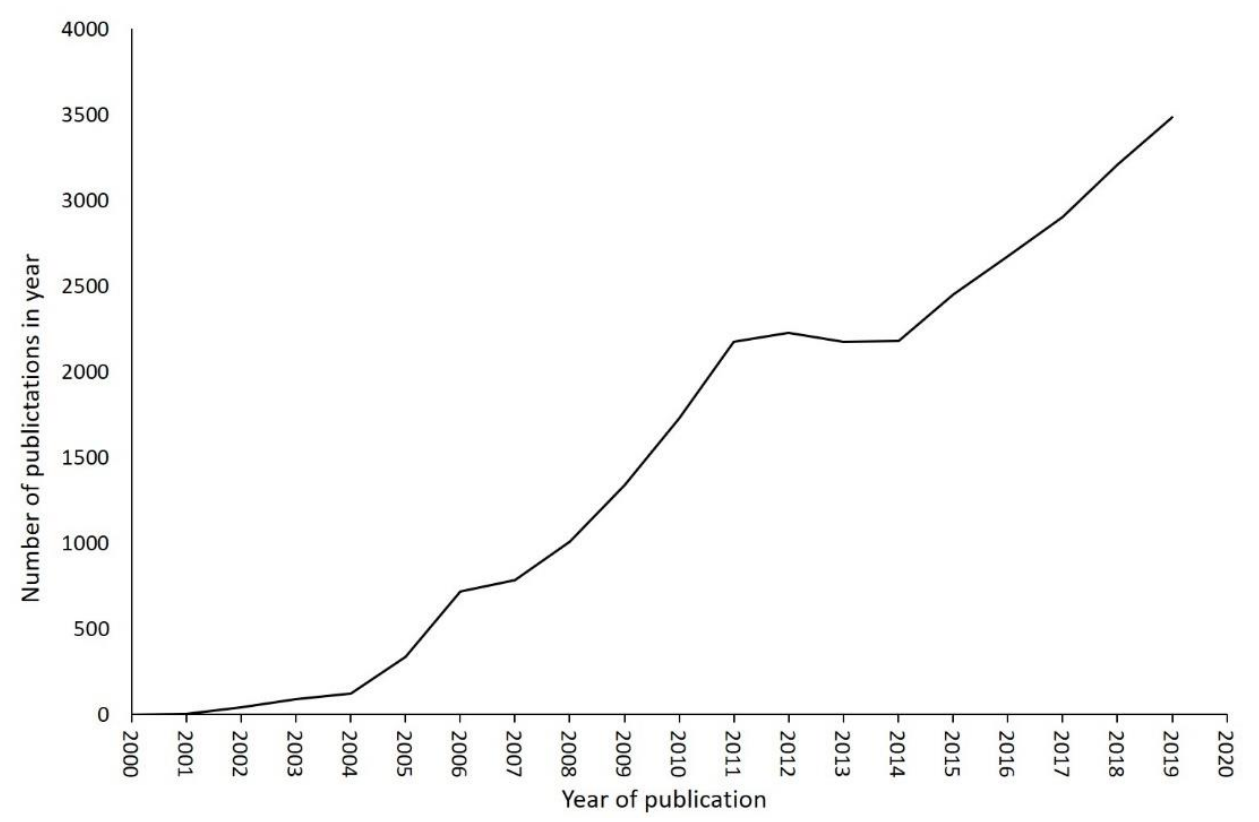

Figure 1: Graph of the number of peer-reviewed articles published with the keyword 'metamaterials' by year [26] showing a dramatic increase in rate of publication from near zero to 3500 papers per year within 20 years.

While various production-ready methods exist for metamaterial manufacturing [3], this paper intends to give the reader a full account of metamaterials which have been produced by AM and focuses on methods and the relationship between the fabrication technique used and the physical properties of the resultant meta-structures. The reader is also referred to comprehensive review papers on optical [27-30], acoustic [31-33] and miscellaneous [34-38] 
properties of metamaterials. Alongside these, a number of review papers that detail numerical methods which inform design methodologies and predict performance characteristics across excitation domains are available [39-41]. This paper unites the critical contributions made in this field with the opportunities for emergent manufacturing techniques in additive manufacturing in order to bring these research communities together.

Additive Manufacturing (AM) or three-dimensional (3D) printing is the technology of building 3D objects layer-by-layer using computer-aided designs (CAD) [42]. Layer-by-layer manufacturing in AM contrasts with conventional subtractive and formative manufacturing. AM starts with CAD software that sends the digital objects (the design) to a printer. The design is then sliced into a predefined number of layers and fabricated sequentially [42]. Hence, this method is well suited to produce nested features and high degrees of structural complexity associated with metamaterials. There are currently more than 20 different recognized AM technologies [43] which are best summarised by the associated ASTM standard into seven discrete groups [4I]. R.F. Housholder patented the first AM technology in 1979 with the U.S. patent office [44] but his work was not commercialized. After almost 4 decades the improvements in the AM resolution and material availability enables researchers to design and fabricate complex structures in robust engineering materials which are not possible via conventional subtractive manufacturing techniques [45].

The capabilities of AM have attracted metamaterial researchers to use this approach as a powerful tool to fabricate a new generation of metamaterials. The possibility of fabricating free-standing complex 3D geometries, low manufacturing costs and direct fabrication from CAD design enhances the design process. This enables researchers to fabricate complex structures with predefined optical, acoustic, and mechanical properties. In this review, the various AM approaches are described only briefly; the reader should refer to comprehensive reviews on AM techniques for a more in-depth understanding of each technique [46,47]. In addition, a review of materials available to additive manufacturing is explored in detail by Bourell et al.[48].

This review contains sections dedicated to specific categories of metamaterials and explores different AM techniques used in the fabrication of these metamaterials. The focus of this paper is the strengths and shortcomings of these techniques with a view to highlighting opportunities for future research. Electromagnetic (EM) and Mechanical (MM) metamaterials are considered based on the various AM techniques used in their fabrication. Conversely, Acoustic Metamaterials (AMMs) are described under their three main types of structure, owing to their mode of operation being very different, and the fact that this needs to be considered during design and fabrication.

\section{Electromagnetic (EM) Metamaterials}

\subsection{Introduction}


Three major breakthroughs shaped the field of EM metamaterials and their applications. The first is the mathematical prediction of negative refractive index by Veselago in 1968 [49]. Second, the experimental realization of negative refractive index materials by Smith et al. in 2000 [50]. Third, the theoretical suggestion of negative index materials and their application to lenses and imaging by Pendry [41].

From a constituent material point of view, EM metamaterials can be classified into either alldielectric metamaterials or metal-dielectric (hybrid) metamaterials. All-dielectric metamaterials are composed of one or more dielectric materials with a large difference between the dielectric constants of neighbouring components [51]. Due to weak interaction with the electromagnetic wave, all-dielectric metamaterials have lower losses compare to metaldielectric metamaterials [52].

Split Ring Resonators (SRRs), illustrated in Figure 2, are one of the most researched structures for metal-dielectric structures. Ferromagnetic metal structures that have negative permittivity and permeability properties can be produced using the structure-dependent properties of the SRR. However, SRR structures are generally considered to be lossy, narrowband and to present complications in practical applications [51].

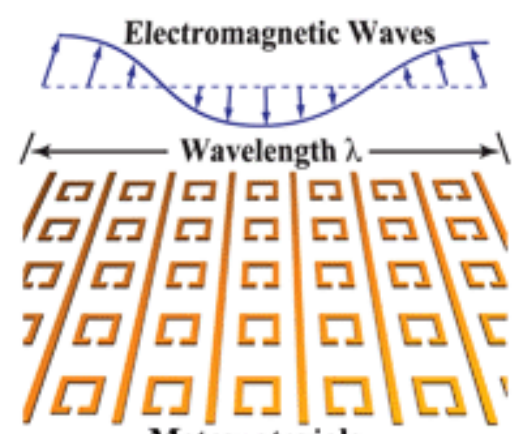

Metamaterials

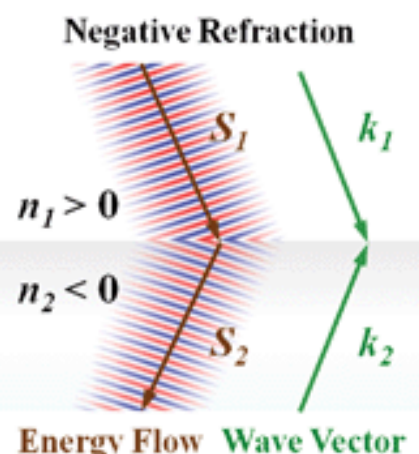

Energy Flow Wave Vector

Figure 2: Schematic diagram of the SRR meta-atoms in comparison to the active wavelength of the metamaterial (left) and the energy flow diagram for negative refractive index material (right) [27].

Photonic Crystals (PCs) possess structure-dependent properties. Therefore, based on the definition presented by the European Union Metamorphose network [53], PC structures are also a class of metamaterials. The defining characteristic that sets PCs apart from other classes of metamaterials is the fact that in PC structures the unit-cell is of the same order to their intended operation wavelength [54]. In contrast, the unit-cells used in other types of metamaterial tend to be far smaller than their operating wavelength [55]. Yablonovitch and John published two seminal papers in describing the physics behind photonic crystals [56,57]. After Yablonovitch proposed 2D and 3D optical bandgap generation in 1987 [56], he tried to prove the presence of optical bandgap experimentally by drilling cylindrical holes in a dielectric block [9]. However, constructing a face centred cubic lattice by drilling cylindrical holes is tedious and inaccurate. Zbay et al. [58] suggested woodpile structures as an effective pattern for the generation of stop bands in 3D PCs. Currently, the woodpile structure is one of the most common PC structures to exhibit a 3D optical bandgap [3]. Ideally the unit-cell of the 
metamaterial (the meta-atom) should be smaller or at the same length scale as the operating wavelength of the metamaterial. Therefore, the resolution needed for the fabrication of these structures is at the upper limit of conventional machining technologies. Consequently, computer-aided machining alongside other subtractive techniques are not suitable for fabrication of such 3D metamaterials.

An alternative to these conventional machining techniques is the use of wafer manufacturing techniques (lithography). Lithographic techniques are inherently 2D patterning techniques that usually suffer from limited build volume, low throughput speed, problems in scalability and high manufacturing costs. In addition, PCB (printed circuit board) manufacturing techniques can be used in the fabrication of metamaterials, such as those used in the microwave region, although PCB fabrication techniques such as computer numeric control milling and chemical etching are limited to 2D and 2.5D surfaces and structures. The PCB substrates also contribute to substantial losses in the metamaterial structure. Therefore, the combination of $2 \mathrm{D}$ structures with a high loss in the substrate greatly reduces the performance of these designs. The use of toxic chemicals in chemical etching is also another key factor that makes such techniques undesirable [5].

Although all-dielectric metamaterials have lower attenuation comparing to hybrid metamaterials, the design challenges and limitations imposed by subtractive manufacturing techniques restricted the use of all-dielectric metamaterials [45]. 3D dielectric unit cells have a superior dielectric response, higher surface area for charge accumulation and thicker current conducting channels that can broaden the metamaterial response to polarisation and angle of the incident field by producing larger local dipole moments within the unit cell [5]. The need for a technique that can fabricate 3D structures with high speed and repeatability fuels the research in the fabrication of metamaterial structures using AM. The design limitations can be relaxed by taking advantage of AM capabilities to fabricate complex freestanding structures directly from a CAD design [46]. Efforts made to produce such structures are explored herewith.

\subsection{Material Extrusion}

Metamaterials with millimetre and sub-millimetre length scales are becoming increasingly important [59], due to the high communication speeds and radar image resolution that are possible. PCs with electromagnetic bandgaps within the millimetre wave region, operating at microwave frequencies, thus have numerous applications. Fused Deposition Modelling (FDM) is one of the main AM techniques being used in the fabrication of all-dielectric metamaterials [51,59-61]. FDM fabricates 3D structures by depositing layers of extruded molten plastic on the substrate. The smallest extruder nozzle which is commercially available is $0.1 \mathrm{~mm}$ in diameter. One of the main advantages of FDM is material availability and the ability of this technique to deposit multiple materials simultaneously. The capital investment and vast open source community has created a remarkable hot bed of innovation in the utility of this technology. 
It is also possible to produce bespoke feedstocks with specific properties for this technique. Thermoplastics used in FDM can be mixed with a wide variety of particles/fibres to produce high dielectric constant materials suitable for the fabrication of PC structures. Using FDM, the structures can be fabricated directly from CAD. Therefore, rapid prototyping/manufacture is possible, and the high level of manufacturing control enabled by AM allows the deliberate introduction of discontinuities in the structure. This can produce a tailored frequency response. FDM resolution is mainly limited to the extrusion nozzle size $(0.1 \mathrm{~mm}$ to $0.4 \mathrm{~mm})$ which can lead to rough surfaces. The high surface roughness of structures fabricated using FDM can suppress the propagation of visible, ultraviolet and infrared light but FDM's resolution and fabrication speed make it ideal for $\mathrm{THz}$ and microwave applications [62].

Advances in FDM have improved our ability to fabricate bespoke parts and to tailor local material properties by graduation of composition. Incorporation of piezoelectric materials, ceramics and metal powders in thermoplastics produce different functional thermoplastic feedstocks for FDM, increasing the range of application of this technique [51,63-66]. Sintering processes can be used to remove the thermoplastic and produce high quality and high dielectric structures using FDM [59].

Producing custom FDM filaments introduces new challenges for the fabrication process. Producing high dielectric constant filament is a time-consuming process, and only limited amounts of ceramic particles can be added to the thermoplastic. The shrinkage caused by sintering and drying process can greatly change the designed parameters, reduce the rod size and destroy the periodicity of the structure. By testing the filaments it is possible to quantify the shrinkage and consider it in the design stage. This technique also suffers from long postfabrication processing, and steps like debinding and sintering which are essential for the fabrication of high dielectric constant structures.

Electron transition from a higher energy band to lower energy band can be used as the source of radiation for different EM frequencies. Due to the lack of molecular resonance in the Radio Frequency $(\mathrm{RF})$ and microwave regions, metamaterials that can operate in these regions become increasingly important [45]. Garcia et al. studied the fabrication process and design optimization of anisotropic structures fabricated using FDM [45]. The effective dielectric constant of the fabricated structures was then compared to numerical simulation. The polycarbonate FDM structures had a dielectric constant of 2.57. By introducing air packets, the dielectric constant of the structure was reduced to 1.85 [45]. Note that the structure-dependent dielectric constant is a critical part of Graded Index (GRIN) lens production that can exhibit total absorption and lens effects $[67,68]$.

All-dielectric invisibility cloaks operating in Microwave $\mathrm{X}$ band (8.6 GHz to $12 \mathrm{GHz}$ ) have been fabricated using FDM [69]. ABS plastic with a dielectric constant of $(2.6 \pm 0.2) \pm 0.01 i$ was used to build the disk-shaped cloaking structure, optimized using the COMSOL optimization solver to minimize the total scattering of the structure. 
Lu et al. [60] and Lee et al. [59] designed and fabricated woodpile structures with an operating frequency of $95 \mathrm{GHz}$ using FDM. In their experiment, structures were fabricated with a rod diameter of $0.4 \mathrm{~mm}$ and a periodicity of $1.6 \mathrm{~mm}$. For their experiment, a mixture of high purity alumina powder $(99.992 \%$, d50 $0.48 \mu \mathrm{m}$, ex Condea Vista, Tucson, Arizona) was used to produce a high dielectric paste suitable for FDM extrusion. The final paste had $12 \mathrm{wt} \%$ [60] and $15 \mathrm{wt} \%$ [59] alumina. Lu et al. suggested that, by changing the processing parameters, it is possible to rectify some of the shortcomings of using custom filaments [60]. They predicted that tensile cracking could be reduced by decreasing the ceramic concentration to below $60 \%$. They also suggested that the drying and sintering processes are important factors in the final fabricated structure. The best result was achieved by using a continuous low heating-rate of 2 ${ }^{\circ} \mathrm{C} / \mathrm{min}$ [60]. Figure 3 shows the structure by Lu et al. and a comparison between the measured and calculated transmittance value for the manufactured structure [60].
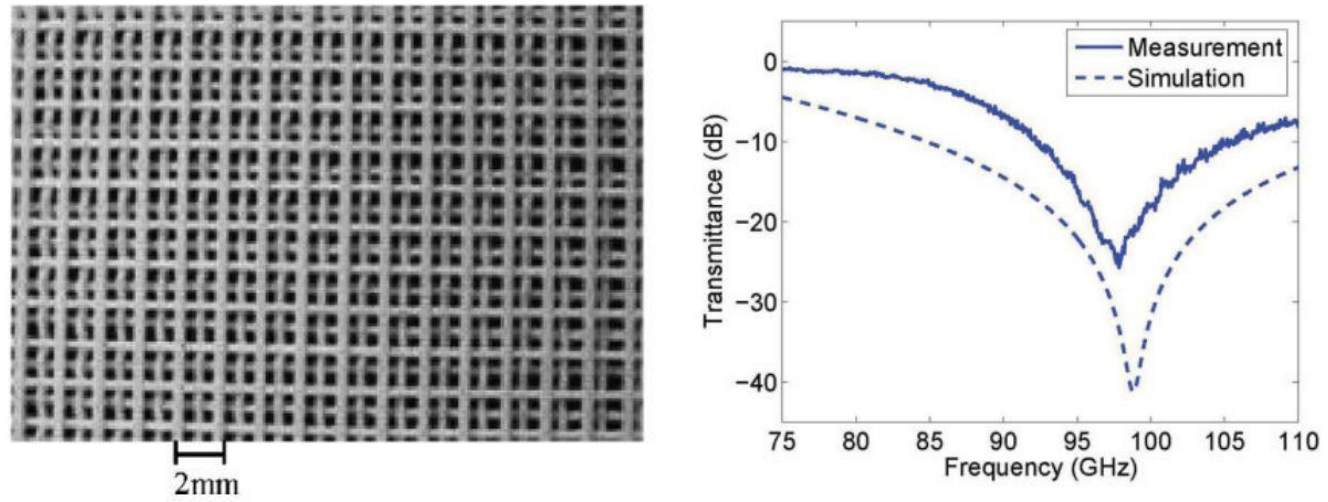

Figure 3: SEM image of a PC fabricated using FDM techniques with a unit cell of $2 \mathrm{~mm}$ and a rod diameter of $0.4 \mathrm{~mm}$ (left). Comparison of the measured and calculated bandgap for this PC structure [60].

Advances in extrusion techniques and the introduction of micro FDM techniques with sub-100 $\mu \mathrm{m}$ resolution have enabled researchers to use this method for fabricating $\mathrm{THz}$ metamaterials. For example, ceramic lattices with a spatial resolution of $<100 \mu \mathrm{m}$ are possible by using $80 \mu \mathrm{m}$ extruders at room temperature [70]. In addition, new filaments using a mixture of ferrite powder and ABS polymer have demonstrated an increase in the permittivity and permeability by increasing the ferrite content [71]. The printed blocks of polymer-ferrite composite had an approximately constant permittivity and permeability in the tested frequency range. By breaking the dependency of material dielectric properties to the operation frequency, the natural design barriers were thus broken.

Multi-dielectric structures have also been fabricated using dual deposition FDM techniques. Isakov et al. [51] demonstrated the possibility of production of a resonant structure by producing a sub-wavelength packet of all-dielectric structures. In their work, they utilized a dual extrusion 3D printer to deposit layers of high refractive index rods (polymer + ceramic particles) beside areas of low refractive index polymer [51]. These structures demonstrated high absorption near $15.75 \mathrm{GHz}$. 
Hybrid metamaterials are also possible by using a cost-effective commercial conductive filament. The conductivity of commercial filament is sufficiently high for microwave applications around $1 \mathrm{GHz}$, and significant enhancement of the dielectric response is possible by designing 3D unit-cells [5]. Unidirectional microwave antennas and omnidirectional fanbeam antennas are possible via FDM. The directionality can be altered by introducing defects into the PC structure, one example with a resonant frequency of $93 \mathrm{GHz}$ achieving $9.3 \mathrm{dBi}$ directivity [61].

By replacing the extrusion head with a syringe, it is possible to extrude higher viscosity liquids, emulsions and pastes with high solid concentrations. Feedstock production is a challenging process. The prepared material should be viscous enough to retain its shape after deposition but it needs to be fluid enough to exit through the nozzle head. The prepared paste must also have a high solid content to avoid cracking and shrinking [72]. $\mathrm{TiO}_{2} \mathrm{PC}$ structures fabricated using this technique demonstrated effective stop bands near $31 \mathrm{GHz}, 38 \mathrm{GHz}$ and $39 \mathrm{GHz}$ [72]. $\mathrm{TbFeO}_{3} \mathrm{PC}$ structures for $\mathrm{THz}$ switching and refraction were also fabricated using the slurry deposition techniques. The all rare-earth PC structure demonstrates the capabilities of this system in the fabrication of orthoferrite optical elements [73].

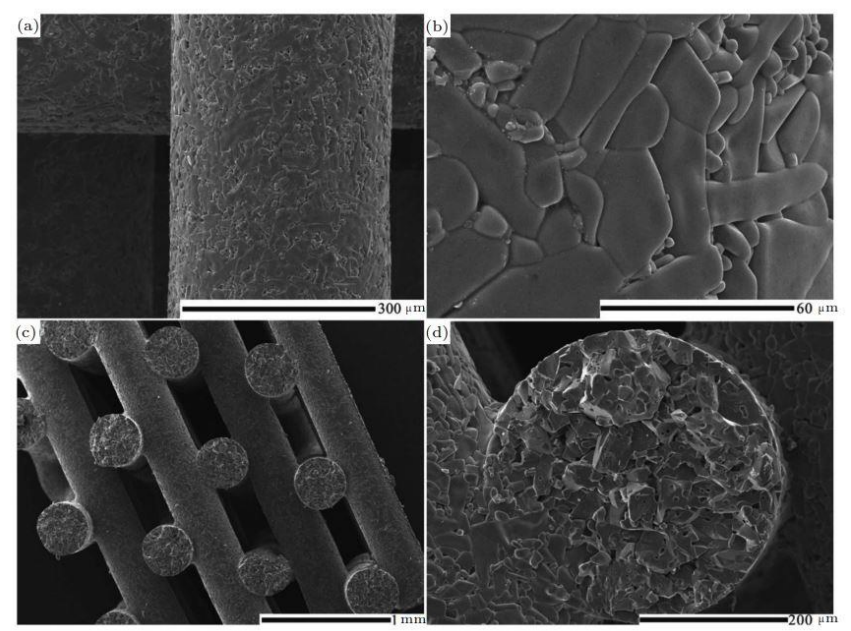

Figure 4: SEM photograph of a 3D extruded woodpile structure fabricated from a ceramic slurry. (a) top view of one rod, (b) microstructure and (c) cross-section of the structure, and (d) a cross-section of one rod [72].

FDM is thus a versatile technique with good material availability for fabrication of millimetre scale photonic crystals, and can deposit more than one type of material.

\subsection{Inkjet Printing}

Inkjet printing is one of the most versatile AM techniques that can produce complex structures on flexible substrates by depositing droplets of inks. The high number of micron-sized nozzles makes this a relatively fast and accurate technique [74]. The selection of available inks that require different curing processes makes the realization of multi-material inkjet-printed 3D structures challenging. For instance, conductive inks are commonly cured using heat treatment that evaporates the solvent and sinters the metallic nanoparticles together. Dielectric inks are 
usually cured using UV radiation and often cannot withstand the sintering temperature of the conductive inks. This issue is particularly important for 3D structures when consecutive layers of materials with intrinsically different prosperities can be printed [74].

The key to the fabrication of PC structures using inkjet printing is the production of jetable and stable ceramic slurries with a high concentration of solids. Reilly et al. [75] studied the effects of different processing parameters such as solid concentration in the slurry, sintering temperature and binder removal rate. By refining the fabrication process, alumina densities of $\sim 98 \%$ were obtained. Similar impurities were found in both powders studied, which suggest that new impurities were not introduced to the alumina during the fabrication process. Reilly et al. [75] used a partially filled cavity method to analyse the loss tangent of the inkjet-printed structure. The Q-factor of 6000 was a major improvement compared to micromachined or milled samples.

Split Ring Resonators (SRRs) are one of the most common metamaterial structures [50,76], noting that hybrid (metal-composite) structures have a stronger interaction with the EM radiation compared to all-dielectric metamaterial structures. Conductive ink printed on a flexible substrate (polymer or paper) is one of the examples of utilization of AM in the fabrication of SRR based electromagnetic bandgap structures for Radio Frequency Identification (RFID) antennas [77]. Using this technique, Lee et al. managed to increase the gain and range of RFID antennas and produce practical RFID tags to be used on flexible surfaces [77]. It is possible to fabricate complex structures by depositing the conductive ink based on the patterns produced by a CAD model. Therefore, complex designs such as RFID antennas with a series of integrated capacitors can be fabricated to minimize the antenna size [78]. Using the smaller RFID antennas Kim et al. printed a metamaterial-inspired, fullyintegrated capacitive sensor using silver ink on the photo-paper [78],[79].

Another example of hybrid metamaterial structures fabricated using inkjet printing is the Jerusalem cross resonator printed onto photo paper, which acts as a flexible metamaterial absorber surface [80]. Silver nanoparticles in solution have been used to print metallic structures and, after sintering the printed structures for 2 hours at $130^{\circ} \mathrm{C}$, the conductivity of the printed parts increased to $1.1 \times 10^{7} \mathrm{~S} / \mathrm{m}$, which is comparable to bulk iron. The intrinsic impedance of the printed Jerusalem-cross resonators was designed to perfectly match the free space impedance, hence minimising the reflection from the printed surface [80]. Other examples of the use of inkjet printing for the fabrication of metamaterials absorber surfaces report $93.5 \%$ absorption at $0.102 \mathrm{THz}$ (Figure 5) [81] and $99 \%$ absorption at $9.21 \mathrm{GHz}$ [82]. As mentioned above, one of the advantages of inkjet printing is the ability of this technique to fabricate structures on different substrates. As an example, Kim et al. [82] covered a cylindrical object with a flexible substrate absorber and achieved higher than $96 \%$ absorption for all polarisation angles. 

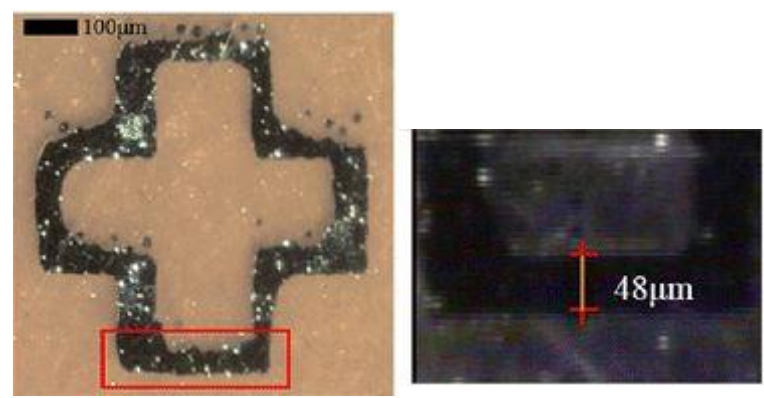

Figure 5: Absorber printed at droplet spacing of $25 \mu \mathrm{m}$ [81].

Inkjet printing has been used in the fabrication of resonance surfaces for biological sensors [83,84]. Kim et al. [83] printed Split Ring Cross Resonators (SRCR) to produce ethanol sensors, as shown in Figure 6, where microchannels etched in polydimethylsiloxane (PDMS) were used to guide the fluids toward the absorber surface of the SRCR. They demonstrated that the resonant frequency of the SRCR increases by $11.2 \%$ when the ethanol concentration increases from $0 \%$ to $100 \%$. The frequency shift for change in ethanol concentration from $20 \%$ to $80 \%$ was linear [83]. In another work, absorber conductive patches printed by Ling et al. were tested by placing inside a polymethyl methacrylate (PMMA) microfluidic channels [84]. The result was $>90 \%$ absorption for both empty and water-filled channels, and the measured resonant frequency decreased from $4.4 \mathrm{GHz}$ to $3.97 \mathrm{GHz}$. The minimum ethanol concentration that can be found using this technique, and the integration of these sensors into a functional sensor unit, are the challenges that should be addressed in future research, but the technique appears very promising.

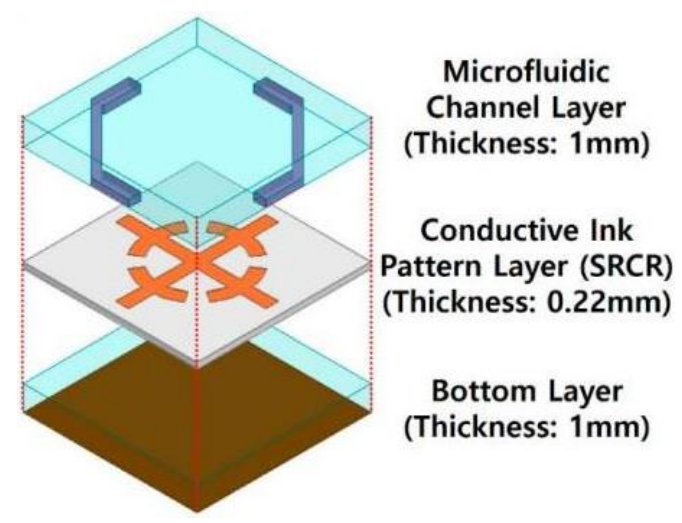

Figure 6: Metamaterial-based ethanol sensor fabricated using inkjet printing [83].

Terahertz metamaterials are often fabricated using advanced micro/nonmanufacturing techniques such as Electron Beam Lithography (EBL) and UV lithography, which require complex deposition and removal techniques performed in a cleanroom environment. This increases the overhead costs of metamaterial fabrication. Due to the flexibility and the ability of Inkjet Printing to use CAD designs for fabrication, this technique can greatly reduce the costs and increase the fabrication speed of Terahertz metamaterials. To compare the EM response of structures fabricated using conventional techniques with inkjet-printed structures, Walther et al. [85] fabricated identical structures using both conventional lithography/etching 
technique and inkjet printing and tested both structures. Broader resonances were observed in the printed structures, which was due to inhomogeneities introduced by the printing process.

Murata et al. [86] have developed an inkjet system that allowed them to fabricate metallic wires of only a few microns on an untreated substrate. The Super-fine InkJet (SIJ) printing technology was used by Takano et al. [87] to fabricate Terahertz metamaterials using silver paste ink. Structures such as a metal wire-grid and an SRR were printed on a high-resistivity silicon substrate using SIJ techniques. Subsequent testing showed good agreement between the Terahertz response of the fabricated structures and their simulated structure. The microprinting technique has also been used in the fabrication of $\mathrm{THz}$ metamaterials with resonant frequencies of $0.305 \mathrm{THz}$ and $0.137 \mathrm{THz}$ for $\mathrm{X}$ and $\mathrm{Y}$ polarized waves [88]. The silver ink used by Kashiwagi et al. [88] reduced the post-processing steps and enabled them to use ordinary printers to print SRR structures on paper, but the printed lines had unavoidable discontinuities.

The low conductivity of printed inks and the need for different curing techniques for conductive and dielectric inks make the process of fabricating hybrid metamaterial structures using Inkjet printing challenging [74]. The progress made in multi print head inkjet printers that can deposit different materials and accommodate different curing methods is a major progress in this area. Despite these challenges, the high throughput and versatility of available inks make this method a favourable AM process for fabrication of low-cost, flexible hybrid structures.

\subsection{Vat polymerization}

Vat polymerization is a category of AM techniques that uses a vat of liquid photoresist to construct objects through layer-by-layer solidification of the resin. This category includes techniques such as Stereolithography (SLA) and Multi-photon polymerization (MPP).

SLA works based on spatially controlled solidification of photoresist (photosensitive material) using a light source. The light crosslinks the monomer and produces insoluble networks [89]. The light source can be a focused laser beam with a specific wavelength that solidifies the photoresist point by point or it can be a LED light reflected by a light modulator to cure a layer of photoresist. The polymerization process occurs near the supporting platform. Bottom-up and top-down approaches are both used in SLA machines. In the top-down approach, the platform is placed at the top of the polymer vat and stage retracts as layers are being built. Therefore, the polymerized structure rests inside the photoresist. In the bottom-up approach, the polymerization occurs through the transparent non-adhesive window at the bottom of the polymer vat. Consequently, the polymerized structure suspends from the support platform outside the resin [90].

MPP is one of the AM techniques that is classified as a vat photopolymerization process [46]. The multi-photon absorption phenomena is a third-order nonlinear effect and the absorption rate decreases sharply outside the focal volume of the objective lens [91,92]. In this technique, simultaneous absorption of more than one photon produces enough energy to initiate the radical 
generation and photopolymerization [93,94]. Figure 7 illustrates this process. Simultaneous absorption of two photons by the Photo Initiator (PI) produce radicals that propagate through the resin and form radicals. Radical monomers are created in the second stage, and in the third stage heavier molecular weight radical polymers are produced from the interaction between radical monomers with other monomers. In the termination process, radical monomers interact with each other and produce non-radical polymers. Due to the low probability of more than one photon coincidence in time and place, a high photon density $\left(\sim 1 \mathrm{TW} / \mathrm{cm}^{2}[95]\right)$ is needed for photopolymerization [96,97]. A high Numerical Aperture (NA) objective lens is used to focus a very tight spot.

$$
\begin{array}{ll}
\text { Initiation } & \text { 1) } P I \stackrel{h v h v}{\stackrel{h}{\longrightarrow}} 2 R \\
& \text { 2) } \dot{R}+M \rightarrow R-M \\
\text { Propagation } & \text { 3) } R-M_{\dot{n}}+M \rightarrow R-M_{\dot{n}+1} \\
\text { Termination } & \text { 4) } 2 \dot{R}-M_{n} \rightarrow M_{2 n}-R
\end{array}
$$

Figure 7: Steps in the two-photon polymerization process [98].

MPP has the highest resolution of all AM techniques, which makes it a promising technique for fabricating micro/nano structures [98,99]. Material availability, limited fabrication area and low fabrication throughput currently limit the application of MPP in practical applications.

\subsubsection{Stereolithography (SLA)}

SLA has been reported as being a useful technique for the fabrication of periodic structures $[67,100,101]$. For example, CAD design allows complex graded structures to be fabricated with a different polymer to air ratio, hence producing a graded-index PC structures. Yin et al. produced a core-shell metamaterial structure by manufacturing graded PC domes using SLA, with a lossy dielectric core to produce an EM "black hole" for $12 \mathrm{GHz}$ to $15 \mathrm{GHz}$ [67].

By introducing ceramic particles into the photoresist, it is possible to increase the dielectric constant and produce high dielectric structures. In woodpile structures, the refractive index gap between air and polymer is a key factor in the creation of bandgaps [102]. In a number of publications, Kirihara et al. [100,103-105] managed to fabricate complex structures with improved dielectric constant by refining the process of dispersing ceramic particles in the photoresist, dewaxing and sintering the fabricated structure. 3D PC structures were fabricated using SLA by dispersing $40 \mathrm{vol} \%$ silica and $10 \mathrm{vol} \%$ titania in epoxy resin. In their experiments, they compared the attenuation in the transmission amplitude in a bulk sample to the PC structure. The silica-epoxy PC structure exhibited a bandgap in the range of 3.2-10.9 $\mathrm{GHz}$, whereas using a titania-epoxy resin exhibited a bandgap in the range of $0.7-6.9 \mathrm{GHz}$. The difference between the band gaps created using identical structures is due to the different 
dielectric constants of the structures [100]. Their experiment proved that, despite using similar rod size and periodicity, a structure with a lower dielectric constant had a bandgap at a higher frequency. Therefore, it's possible to produce a PC structure that performs well within a specific frequency band, not only by changing the structure feature sizes, but also by changing the material dielectric constant [100].

By increasing SLA resolution and fabricating PCs with micro-diamond lattice structures, the operation frequency of PCs has been pushed into the THz region [104]. By introducing defects in the design file, it is possible to tailor further the structure's response to a specific EM frequency. Kirihara [106] managed to produce sharp transmission peaks inside the photonic bandgap of PC structures by introducing defects. Indeed, a complete $\mathrm{SiO}_{2} \mathrm{PC}$ structure has been fabricated by dispersing $50 \mathrm{vol} \%, \mathrm{SiO}_{2}$ in the photoresist and dewaxing/sintering the SLA manufactured structures at $600{ }^{\circ} \mathrm{C} / 1400{ }^{\circ} \mathrm{C}$ [107]. During this process, the PC structure shrank by $24.5 \%$ in $\mathrm{X}-\mathrm{Y}$ directions and $26 \%$ in $\mathrm{Z}$ direction, but the overall form of the diamond lattice structure was preserved. Chen et al. [107] studied the structures using an X-ray diffraction method and reported that the fabricated structures were made out of pure $\mathrm{SiO}_{2}$ ceramic with a mass density of $2.2 \mathrm{~g} / \mathrm{cm}^{3}$ ( $99 \%$ of the theoretical mass density). The lattice constant of the fabricated structure reduced to $380 \mu \mathrm{m}$. Therefore, the aspect ratio between the dielectric rods and the lattice constant reached 1.31. The full $\mathrm{SiO}_{2} \mathrm{PC}$ structure had a dielectric constant of 3.5 and produced a full photonic bandgap at a frequency close to $470 \mathrm{GHz}$ along with different crystallographic directions [107].

SLA has also been used to produce epoxy moulds for ceramic PCs. The inverse PC structure is designed using CAD and fabricated using an SLA 3D printer. Yin et al. used this technique to fabricate PCs composed of $\mathrm{SiO}_{2}-\mathrm{TiO}_{2}$ ceramic [101]. In their experiment, a ceramic slurry was poured into the cast after preparation of the epoxy mould. High packing density was then achieved inside the mould using ultrasonic vibration. The diamond PC structure fabricated using the inverse moulding technique demonstrated up to $30 \mathrm{~dB}$ attenuation around $17 \mathrm{GHz}$ [101]. To produce broader bandgaps in PC structures, Liang et al. [108] used ceramic slurries with two different ceramic concentrations (55 vol.\% and 60 vol.\%). By producing a gradient in the dielectric constant of the PC structure, the bandgap width increased by $117 \%$ using the higher ceramic concentration.

A practical carpet-cloak metamaterial structure based on gradient index phenomena has been fabricated using SLA [109]. The gradually-changing unit cell increases the complexity of the design and introduces new challenges in the fabrication of this structure. This gradual change in the filling ratio produces changes in the refractive index of the structure while minimizing EM wave scattering.

Combining SLA with other techniques is an additional approach for the fabrication of composite structures. Rudolph and Grbic [110] demonstrated a negative refractive index medium built by coating SLA fabricated cubes with copper and soldering capacitors to produce a negative refractive index at $1.5 \mathrm{GHz}$. Also, Wu et al. [111] combined micro-SLA with sputtering deposition to fabricate $30 \mu \mathrm{m}$ thin metallic wires with $120 \mu \mathrm{m}$ spacing. The plasma 
frequency of the fabricated structure was tested using Fourier transform infrared (FTIR) measurements, and a plasma frequency of $0.7 \mathrm{THz}$ was measured for the fabricated structure.

\subsubsection{Multi-Photon Polymerization (MPP)}

The true 3D nature of MPP has allowed the fabrication of free-standing complex geometries to produce the sub-wavelength symmetry that mimics crystal lattice structures. Woodpile structures are the most well-known pattern used in the generation of optical bandgaps [112]. The design freedom that AM brings to metamaterials can be understood in structures like spiral photonic crystals, which are not possible using conventional manufacturing techniques $[112,113]$. Chiral PC structures are one of the most promising designs that can produce effective bandgaps with a low refractive index contrast [114]. The chiral PC structures are fabricated through shifting the rods by $360 / n$ degrees each layer when $n$ is the number of layers in the PC structure [115]. Using MPP, which is a high-resolution mask-less manufacturing technique, it is possible to fabricate such chiral PC structures (see Figure 8), which have a higher mechanical stability than laterally-disconnected structures [115]; further, bi-chiral photonic crystals fabricated using SU-8 photoresist demonstrated the lower directional dependency compared to uniaxial structures (see Figure 9) [114].

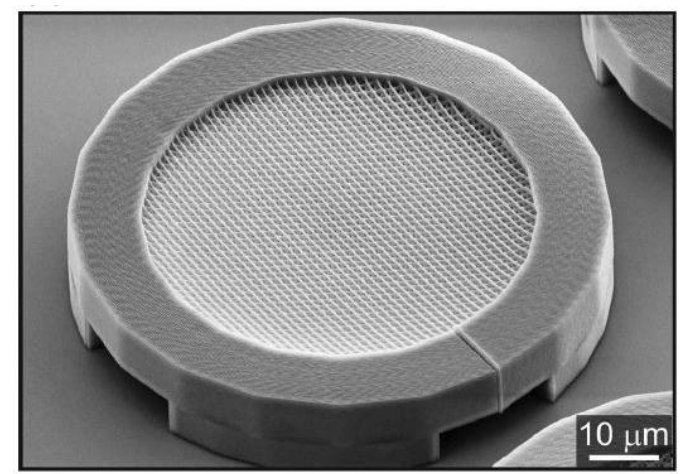

Figure 8 SEM image of chiral PC structure with a layer thickness of $1.32 \mu \mathrm{m}$, a rod diameter of $300 \mathrm{~nm}$ and a lattice constant of $1.2 \mu \mathrm{m}$. The fabricated structure is made of 24 layers with an open window of $60 \mu \mathrm{m}$. The thick wall around the structure decreases sample bending and deformation due to stresses and shrinkage during the polymerization and development process [115]. 
a)

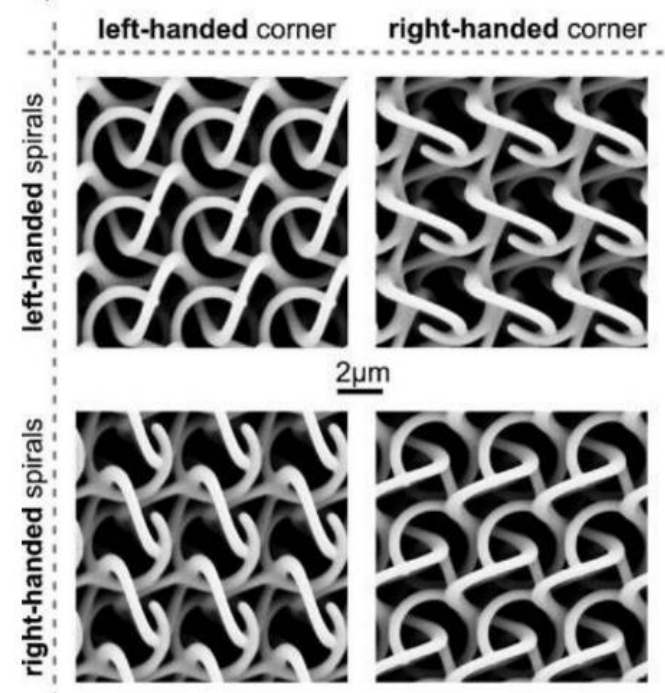

b)

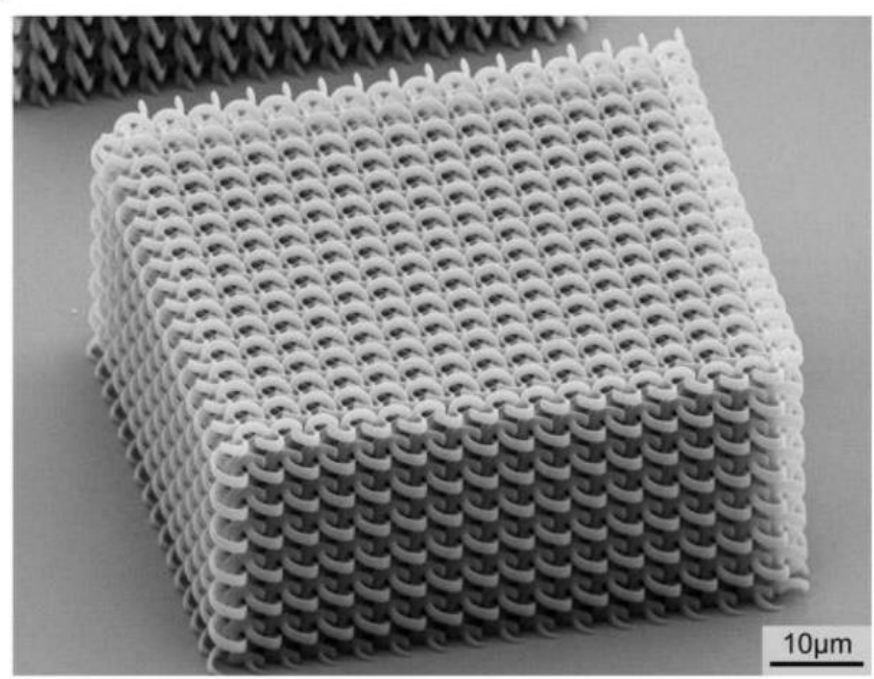

Figure 9: SEM photographs of a bi-chiral photonic crystal, showing a) top view of different arrangements for photonic crystals, and b) $60 \mu \mathrm{m}$ by $60 \mu \mathrm{m}$ right/left photonic crystal fabricated with a lattice constant of $4 \mu \mathrm{m}$ using MPP [114].

Commercial organic photoresist (SCR-500 Japan Resin Company) has been used in the fabrication of PC structures with pitches between $350 \mathrm{~nm}$ to $500 \mathrm{~nm}$. In one example, a 40layer PC structure exhibited an attenuation of 50\% in the near-IR spectral region [116]. Using inorganic-organic polymers, it is possible to fabricate PC structures with higher thermal and mechanical stability. Post-fabrication thermal treatment of PC structures fabricated using inorganic-organic material decreases the unwanted defects and improves the surface roughness of the polymerized structure, in turn decreasing the scattering losses of the structure [117]. Holographic MPP has been investigated for increasing the MPP fabrication area [102] and 200 $\mu \mathrm{m} \times 200 \mu \mathrm{m} \times 20 \mu \mathrm{m}$ samples which contained 40 layers of in-plane rods have been reported. Serbin et al. investigated the properties of woodpile structures fabricated using Ormocer (an inorganic-organic polymer) in the near-IR spectral region [112]. To be able to benefit from the ability of MPP system to fabricate structures with at a range of resolutions, Fernandez and Coninck [118] developed a multiresolution MPP system that uses a new slicing algorithm. The complex slicing and contouring technique used in their work separated areas that require high resolution from the rest of the structure. Therefore, by manipulating the processing parameters, low-resolution areas fabricated much faster than the conventional MPP and the overall fabrication speed improved [118].

Regardless of improvements made in this field, MPP fabrication speed and the limited field of view of high NA objective lens restrict the applications of MPP for the fabrication of mm scale structures. In order to be able to fabricate millimetre-sized structures, the MPP system should use a high-speed laser scanning system accompanied by a piezoelectric stage. 2D Galvonometer mirrors can be used for high-speed laser scanning which can increase the fabrication speed up to $100 \mathrm{~mm} / \mathrm{s}$. A high NA objective lens limits the field of view of the objective lens and the laser scanning system can only fabricate inside this field of view. The 
piezoelectric stage should be used to align the new fabrication area with already existing patterns.

The dielectric gap between commercial MPP resins and air is not wide enough to produce complete bandgaps. It is reported that a refractive index difference of 1.9 is required for the generation of a complete stop-band [112,119]. Therefore, customized resins with a high dielectric constant must be used to produce effective PC structures [112]. There are several approaches to resolving the dielectric gap challenge. One example is the use of $\mathrm{As}_{2} \mathrm{~S}_{3}$ chalcogenide glass, where a thin film is deposited on the substrate using thermal vapour deposition. Using the high refractive index of $\mathrm{As}_{2} \mathrm{~S}_{3}$ (2.45 to 2.53), the MPP fabricated PC structure exhibited a Photonic Bandgap (PBG) with a 3.5\% gap/mid gap ratio [119].

Due to the complex nature of MPP resins, the production of new MPP resins with a high refractive index is time-consuming and expensive [120]. A less complicated approach toward fabrication of high refractive index structures using MPP is to dope commercially available resins with high refractive index particles (such as $\mathrm{TiO}_{2}, \mathrm{ZrO}_{2}, \mathrm{PbS}$ or $\mathrm{ZnS}$ ) [96,121,122]. This mixture can be considered as a single material with effective material properties calculated using effective medium theory [123]. High refractive index and low optical losses in the visible spectrum make $\mathrm{TiO}_{2}$ a very promising material for fabrication of PCs. Doping the MPP photoresist with $\mathrm{TiO}_{2}$ and manufacturing the woodpile structure has been demonstrated by Houbertz et al. [124]. In their experiment, they reported that the high optical absorption of $\mathrm{TiO}_{2}$ in their fabrication wavelength decreased the fabrication speed. Titanium-doped acrylate monomers and oligomers were also used for fabrication of 3D diamond structures using MPP. Duan et al. demonstrated the generation of titanium dioxide $\left(\mathrm{TiO}_{2}\right)$ nanoparticles in the 3D structure and presence of PBG using $\mathrm{TiO}_{2}$-doped resin [125].

Pure metal oxide PC structures can be realised by thermally treating the PC structure at $500{ }^{\circ} \mathrm{C}$, which increases the refractive index of the structure to 2.1 in the visible region [124]. Incorporation of quantum dots into 3D PC structures was another step in the fabrication of optically active devices using MPP. By doping the photoresist with the quantum dots, or by infiltrating the quantum dots using the MPP fabricated PC structures, it is possible to control the spontaneous emission of the quantum dots. This is of interest in quantum optics, energy harvesting and optical communications [126]. Nonlinear Optical (NLO) silica sol-gel is another material that has attracted much interest due to its stability and low cost. Farsari et al. [122] demonstrated the fabrication of 3D photonic crystals using a photosensitive sol-gel material that contains NLO chromophore disperse red 1, where the synthesized resin demonstrated little shrinkage or distortion during polymerization.

Waveguide structures based on PCs provides the ability to guide the light around radial paths whose radii are only several times that of the wavelength of the propagating light; the resulting low losses are important in optical chips and optical computation [99]. Such structures are constructed by carefully introducing defects in the PC structure. Woodpile structures with line defects that represent waveguides with sharp bends have been fabricated using lithographic 
techniques [127-129]. However, lithographic techniques are generally considered as 2D patterning techniques and they are tedious, expensive and complicated [99]. It is possible to produce PC structures using colloidal self-assembly but introducing defects in the desired area is not yet possible. Note that Lee et al. [99] used a combination of direct laser writing and selfassembly to generate waveguides inside the colloidal crystal.

Structures fabricated using MPP can also be used to produce a negative mould for fabrication of PC with high refractive index materials [130]. It is possible to generate structures with sufficient refractive index contrast by backfilling the structure fabricated using MPP with a high dielectric constant material and removing the MPP structure using calcination or other methods [131,132]. Inverse silicon woodpile structures have been fabricated by using two different phase masks to fabricate large area good quality woodpile structures. These structures were subsequently coated with a layer of $\mathrm{Al}_{2} \mathrm{O}_{3}$ using Chemical Vapour Deposition (CVD) and layers of Silicon using atomic layer deposition. After depositing the silicon, Hydrofluoric acid was used to remove the polymer and $\mathrm{Al}_{2} \mathrm{O}_{3}$. The periodicity of the phase masks used during MPP was $600 \mathrm{~nm}$ and the Si inverse woodpile structure with a fill fraction of $18 \%$ demonstrated a complete bandgap [131]. CVD has also been used for depositing silver on MPP structure and effective bandgap centered at $100 \mathrm{THz}$ has been achieved [133]. MPP-fabricated structures have also been employed to produce silicon photonic bandgap structures using a double negative method (double moulding) [11]. In this technique, the polymerized structure was removed after being backfilled by $\mathrm{SiO}_{2}$. The resultant structure was used as a mould for the actual structure. To produce the final silicon PC structures, the silica mould was back filled using silicon and the mould was removed using hydrofluoric acid [11].

MPP-fabricated structures have also been used as a template for highly-refractive $\mathrm{TiO}_{2}$ structures. Serbin et al. [95,112] used SU-8 (an epoxy-based negative photoresist) to fabricate woodpile structures. After completing the fabrication process the structure was immersed in $80 \%$ titanium-IV-isopropoxide and $20 \%$ ethanol for one hour under helium. The structure was then exposed to air for 12 hours to form a solid $\mathrm{TiO}_{2}$ layer. The polymer structure was removed by heating to $600^{\circ} \mathrm{C}$ for 4 hours. The major drawbacks of this technique are the long postfabrication processing and the surface roughness of the coated $\mathrm{TiO}_{2}$ layer. Note that the use of MPP for mould production or fabrication of inverse woodpile structure that exhibits PC behaviour increases the challenges of $\mathrm{PC}$ fabrication by dramatically increasing the number of post-fabrication steps. This technique also magnifies any distortions in the polymer structure which leads to nonuniform surfaces [131].

Even though MPP is generally a single material system, a network of high and low dielectric packets can be fabricated using MPP [134,135]. In their experiments, the substrate was immersed in the first photoresist and areas associated with the first resin was solidified using the femtosecond laser. After completing the fabrication process of the first material, the sample was developed, and then cleaned to avoid cross-contamination. To introduce the second material, the structure was placed in the second photoresist and further fabrication was carried out on the previously-developed MPP structures Using this technique, it is possible to fabricate 3D structures with a high resolution and with graded dielectric properties, but the fabrication 
process is very time-consuming and error-prone. Multiple stages of sample development and realignment decrease the efficiency and accuracy of this technique.

High dielectric particles encapsulated in low refractive index polymer can behave as a photonic crystal. It is possible to trap and transport particles and lock them in a polymer matrix using optical tweezers to assist MPP. A well-defined 3D array of high dielectric particles fixed in a low refractive index polymer can then be considered as a 3D photonic crystal [136]. MPP can also be used to fabricate hybrid (metal-dielectric) metamaterials. MPP fabricated metaldielectric structures with high spatial resolution can pave the way for visible metamaterials and photonic crystals [137]. Considerable efforts have been made to fabricate metallic structures using MPP [138]. These efforts can be divided into two major categories: direct deposition and post-functionalization [132]. There are three main strategies for direct deposition of metallic microstructures:

1) Direct metallic salt reduction $[139,140]$.

2) Photoreduction of metallic salt in the polymer matrix [137].

3) Simultaneous photopolymerization and photoreduction metallic salt [138].

Kawata and coworkers demonstrated the fabrication of 3D silver structures using photoreduction of silver salt [139,140], and Fourkas and co-workers demonstrated solution phase deposition of Iron and Gold [141]. Typically, solution-phase deposition has been shown to produce isolated metallic structures with generally poor conductivity [132]. It is possible to fabricate composite structures by using a combination of commercially available MPP photoresist and metal salts. To achieve high-resolution composite structures, it is essential to use two-photon absorption dyes which are both expensive and limiting [142]. Chaudhary et al. [142] demonstrated the fabrication of composite (metal-polymer) structures with a minimum feature size of $390 \mathrm{~nm}$ without using dyes and fabricated metal-polymer gratings. This is shown in Figure 10 for different spacing and sizes. Using Lucirin-TPOL photo initiator (Ethyl $(2,4,6-$ trimethylbenzoyl) phenyl phosphinate) and introducing $10 \mathrm{wt} \%$ gold precursor they managed to increase the diffraction efficiency of gratings fabricated by MPP from $10 \%$ to above $60 \%$ [142].

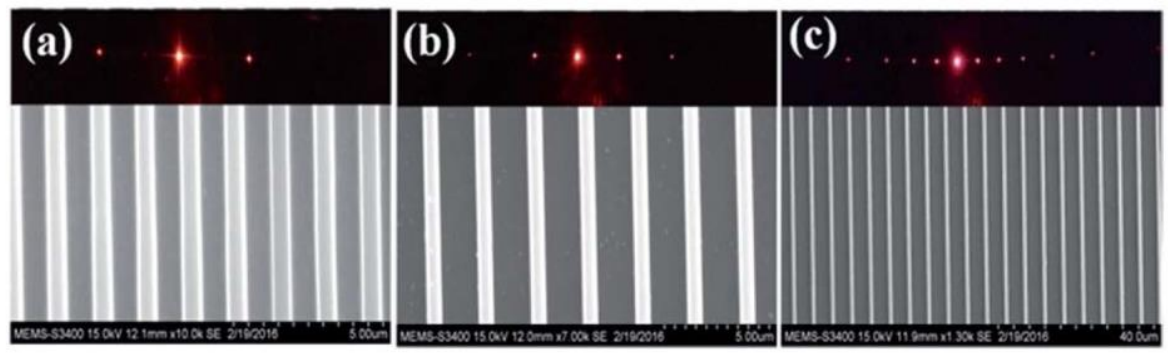

Figure 10: Diffraction gratings fabricated using 5 wt\% gold precursor-loaded polymer. The diffraction pattern of $712 \mathrm{~nm}$ red laser was generated by structures with periodicities of (a) 1.5 um, (b) $2.5 \mu \mathrm{m}$ and (c) $5 \mu \mathrm{m}$ [142].

Truly 3D free-standing metallic structures have been fabricated by covalently bonding photo reducing dyes to nanoparticles in the mixture of silver salt and sacrificial host polymers [137]. 
A femtosecond laser has also been used for fabrication of highly conductive subwavelength metallic lines [143]. By using the femtosecond laser to write in a gold precursor doped photoresist, line widths a low as $100 \mathrm{~nm}$ are posssible, with conductivity within an order of magnitude of the bulk metal. Such structures can be used for fabrication of different optical structures.

It is possible to produce 3D periodic conductive structures from MPP fabricated structures by using metal coating techniques [144-148]. Such hybrid techniques have been used for fabrication of periodic woodpile structures [149], U-shaped resonators [146] and perfect absorbers [147]. The main advantage of electroless plating is the quality of deposited metal which has conductivity comparable to the bulk material. Using CVD, a thin metallic layer is deposited on the substrate as well as the polymer structure that limits the functionality of the sample. As is demonstrated in Figure 11, an intermediary step can be used to mechanically remove the fabricated microstructure from the coated substrate and place the coated structure on a new substrate [149].

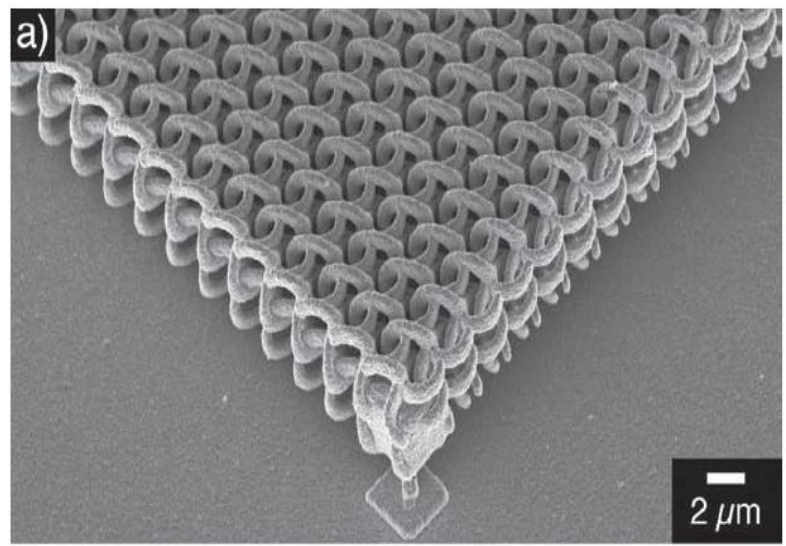

b)

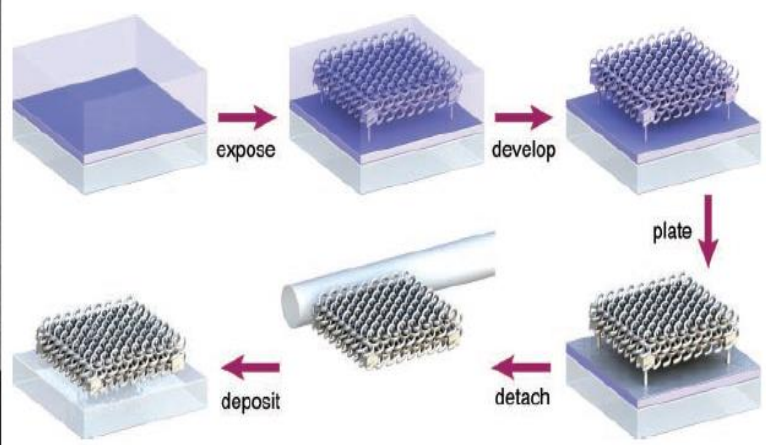

Figure 11: a) SEM image of a MPP-fabricated bi-chiral photonic crystal, together with b) the process diagram for fabrication, plating and redisposition of the structure on a new substrate [149].

Post-functionalization is another promising method for selective metal coating of specific areas of design. Adding metal receptive functional groups to the MPP photoresists enables selective metal coating of polymer structures, as demonstrated by Kawata et al. [150] who fabricated polymer structures with the non-activated and activated polymers (amide-containing polymers). Completing the plating process, the non-activated polymer structures stayed transparent but that regions containing activated polymer changed colour and accepted the silver coating.

Yan et al. [151] showed that treating structures with a radio-frequency plasma helped to reduce the number of post-fabrication steps and increase the quality of the metallic layer deposited on an MPP SU-8 structure. The surface treatment helps the SU-8 structure, fabricated on a Si substrate (e.g. as split-ring resonator structures, see Figure 12) to be coated preferentially with high conductivity and low surface roughness. 

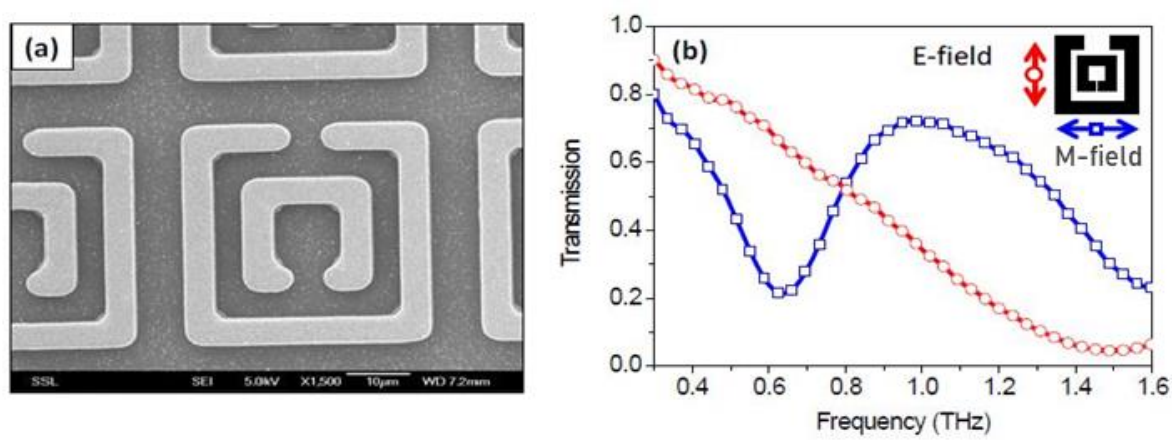

Figure 12: (a) SRR structure fabricated using SU-8 on a silicon substrate. The structure was treated with $R F$ plasma prior to silver coating so that the high-quality metal coating was only applied to the SU-8 structures. (b) Transmission spectra for electric field parallel and perpendicular to the SRR gap show an LC resonance dip at $0.64 \mathrm{THz}$ [151].

Another approach in the fabrication of composite metal-polymer structures using MPP is to combine this technique with Laser-Induced Transfer (LIT). It is possible to deposit high-quality spherical nanoparticles on specific areas of a design. Kuznetsov et al. demonstrated deposition of $200 \mathrm{~nm}$ to $800 \mathrm{~nm}$ nanoparticles on MPP fabricated structures [152]. In their experiment, a gold-coated (10 nm to $60 \mathrm{~nm}$ thick) glass slide was placed a distance of $10 \mu \mathrm{m}$ from the coverslip (substrate). The femtosecond laser then melted the gold and backwards-transferred the molten metal to the substrate. This approach produced high-quality spherical gold nanoparticles, which was not previously achievable, and allowed the particles to change shape [152]. Note that the femtosecond laser is used for both LIT and polymerization, and therefore the LIT process cannot be done in the presence of photoresist.

Infrared concentration using a Gradient Index (GRIN) lens is an attractive solution to increase the photometric performance of sensors by concentrating the light into a small area. Moughame et al. [153] utilised MPP to fabricated small GRIN lens (volume 1.5 $\lambda^{3}$ ) with air gaps from $\lambda / 20$ to $\lambda / 8$ to concentrate mid-infrared light [153]. The GRIN lens fabricated in this experiment achieved an intensity increase of up to 2.5 times. During this experiment, a homemade tri-acrylate polymer was used to fabricate the metamaterial structure, but they hypothesised that by using higher refractive index material like silicon, high-quality GRIN lenses could be realised [153].

Using MPP in the fabrication of composite structures is a challenging task. Using direct deposition techniques such as metal-salt reduction produces high-resolution 3D structures but results in poor metal quality and disconnected metal particles [132]. Using hybrid techniques increase the fabrication steps but introduces new difficulties.

Metallic wire-grid linear polarizers are one of the most well-established structures and extending these structures to 3D can solve some of the limitations of the linear polarizer. Gansel et al. [154] used a combination of multiphoton polymerization and chemical vapour deposition to fabricate a 2D array of gold helix structures that blocked the circularly polarized light with the same handedness as the helices. Figure 13 (a)-(c) shows the different steps taken in the 
fabrication of gold helix arrays. During the experiment, the glass substrate was coated by 25 $\mu \mathrm{m}$ of ITO, which acted as the cathode for metal deposition. The substrate was then spin-coated with a $10 \mu \mathrm{m}$ thick layer of a positive photoresist, and MPP utilized to write the helices. In the development stage, only sufficiently-exposed areas were removed and air helices were created. By placing the structure in an electrochemical cell, gold was deposited in the air helices and the unexposed polymer was removed using air plasma. Freestanding metallic helices with a lattice constant of $2 \mu \mathrm{m}$ have been fabricated using this technique (Figure 13 (d)-(f)). Although the use of positive photoresist limits the resolution achievable by MPP, the resolution can be increased using stimulated emission depletion-assisted MPP. This allows more accurate helices to be fabricated with more than 3 intertwines [155].

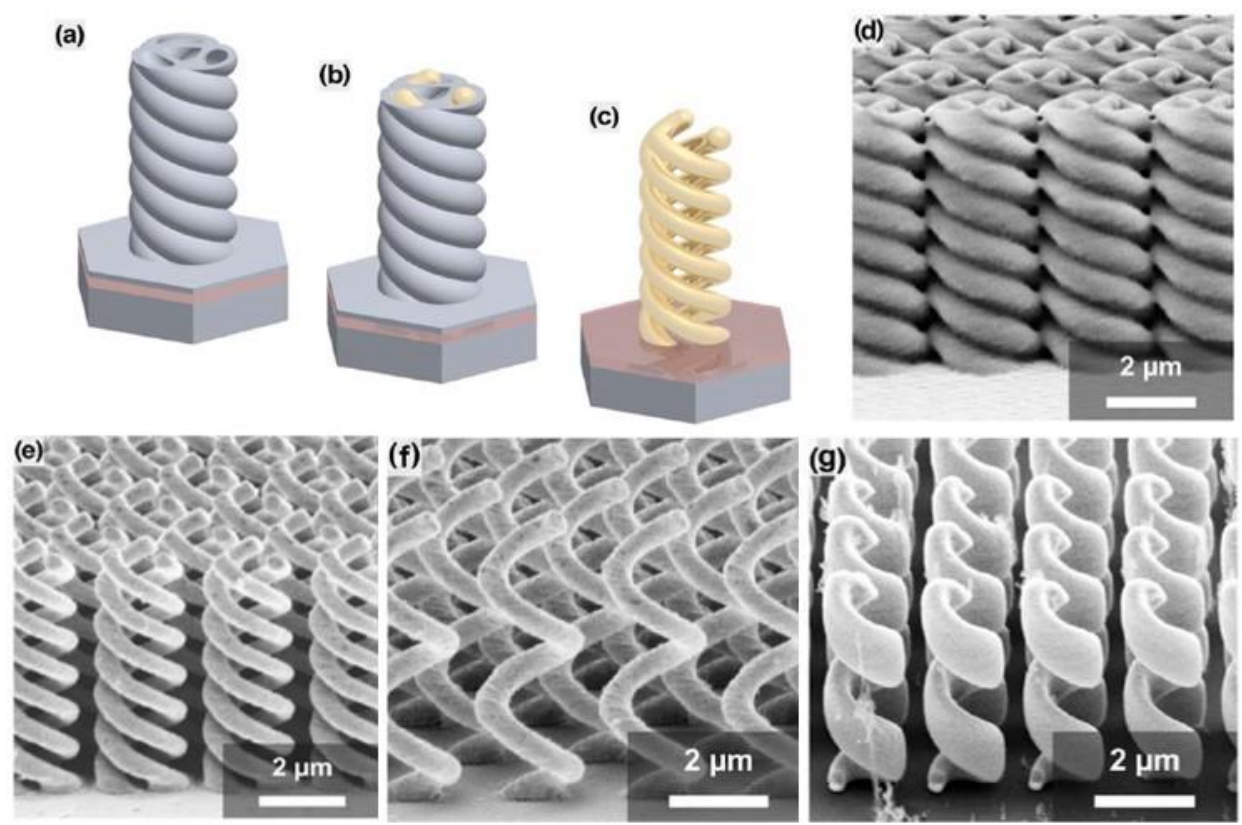

Figure 13: Metallic helices fabricated using a combination of stimulated emission depletion assisted MPP and electrochemical metal deposition. (a) to (c) diagram illustrates different steps of fabrication; (d) SEM picture of polymer shells; $(e)$ and $(f)$ show arrays of metallic helices fabricated using negative photoresist, and $(g)$ shows an array of metallic helices fabricated using positive photoresist [154].

\subsection{Other techniques}

The classification of AM techniques is complicated due to the rapid expansion of the field. In some cases, an AM technique can be placed into more than one category. In this section, we present some of the AM techniques that were used in the fabrication of EM metamaterials. These techniques are either less well-established or have limited application in fabrication of the EM metamaterials.

\subsubsection{Laser Chemical Vapour Deposition (LCVD)}


LCVD uses a focused laser beam to locally heat the substrate and drive the chemical vapour deposition reaction [156]. LCVD has been used in the fabrication of rods, springs and micro tweezers [157]. Therefore, it is possible to use LCVD to fabricate high dielectric structures, and hence to fabricate PC structures [60]. Wanke et al. used a mixture of oxygen and trimethylamine alane $\left[\left(\mathrm{CH}_{3}\right)_{3} \mathrm{~N} \cdot \mathrm{AlH}_{3}\right]$ to grow $40 \mu \mathrm{m}$ aluminium oxide rods [158]. These structures showed transmission minima centred around 2 and $4 \mathrm{THz}$ [158].

\subsubsection{Aerosol jet printing}

High fabrication cost of components with a resonance frequency in $\mathrm{THz}$ region limited the widespread adaptation of devices that operate in the region from $100 \mathrm{GHz}$ to $10 \mathrm{THz}$ [159]. Aerosol Jet Printing (AJP) utilizes a low viscosity inks in the range of 1-25000 cP to fabricate micron-sized feature sizes [160]. The feature sizes achieved using AJP addressed the gap between high-resolution techniques like vapour deposition and low-resolution techniques like screen printing. Comparing to inkjet printing, AJP can be used with higher viscosity inks and the features produced using AJP have higher resolution. Metamaterial-inspired $\mathrm{THz}$ filters fabricated using AJP with operation frequencies of $230 \mathrm{GHz}, 245 \mathrm{GHz}$ and $510 \mathrm{GHz}$ demonstrates the capability of this system in the fabrication of filters, mixer and absorbers functioning in THz region. The AJP fabricated structures were compared to their lithographic counterparts and they achieved comparable performance [159].

\subsubsection{Powder bed fusion}

Powder bed fusion techniques rely on the utilization of an energy source to melt and fuse a thin layer of metal powder to fabricate complex structures layer by layer. Selective Laser Melting (SLM), Selective Laser Sintering (SLS) and Electron Beam Melting (EBM) are the most notable techniques in this category. There are 3 major steps in powder bed fusion techniques. First, a layer of powder is spread on the build platform. Second, a laser/electron beam fuses the first layer to the build platform. Third, a new layer of fresh powder is deposited on the retracted platform. Step two and three are repeated until the structures are fabricated layer by layer. The powder particle size and laser power density control the melt pool size and the resolution of the system [161].

Additive manufacturing of metallic parts enables researchers to fabricate complex metal components with high precision and repeatability. SLM has been used for fabrication of microwave sources based on slow-wave structures [162]. Microwave sources operate based on the conversion of the electric beam energy into radiation by coupling the AC beam current into an interaction circuit. By replacing the interaction circuit with a metamaterial structure, new types of microwave sources have been realized. French and Shiffler [162] demonstrated that metallic helix structures fabricated using SLM can be used in the production of high power microwave sources While the structured finish and surface roughness of the SLM parts were lower than parts made with conventional machining, the design freedom, fast turnaround and low cost of AM parts enabled them to manufacture and test the behaviour of these structures 
for the production of microwave sources. Due to high interaction between EM waves and metallic structures these structures exhibit high losses. Therefore, the resultant metallic metamaterials are not very efficient in the optical region.

\subsubsection{Laser Rapid Prototyping}

Laser Rapid Prototyping (LRP) was one of the earliest forms of additive manufacturing technologies that were used for fabrication of photonic crystals with a bandgap in the millimetre range [163]. LRP uses a photocurable ceramic paste to produce 3D structures with properties comparable to other ceramic manufacturing techniques (injection and pressing). In this process, a paste feeder and a sweeper blade apply a fresh layer of photo-curable ceramic to build complex structures layer by layer.

\subsection{Summary}

As demonstrated in Figure 1, metamaterial research is expanding and focusing on the utilization of the novel properties of metamaterials. The expansion in the metamaterial research is partly due to new manufacturing and characterisation techniques developed in the past few decades [164]. Figure 14 summarises some of the most important works done in this field and demonstrates the feature sizes achieved using respective fabrication methods. It is clear that increasing the fabrication resolution (i.e. decreasing the minimum feature size) increases the application frequency of the metamaterial. The relationship between the metamaterial feature size and the operation frequency depends on the type of metamaterial. The PC structures operate at the wavelength comparable to their periodicity and metamaterials operate in wavelengths greater than the constituting unit cell of the structure.

To summarise this section, Figure 14 presents a diagram where the methods discussed are listed with respect to operating frequency and feature size. It is clear that MPP has the capability to fabricate structures with submicron feature sizes. The operation frequency of these structures can be up to $272 \mathrm{THz}$ which is close to the theoretical limit that metals behave as perfect conductors [165]. Inkjet printing and FDM have a relatively lower resolution but they can be used for fabrication of practical, large scale structures. 


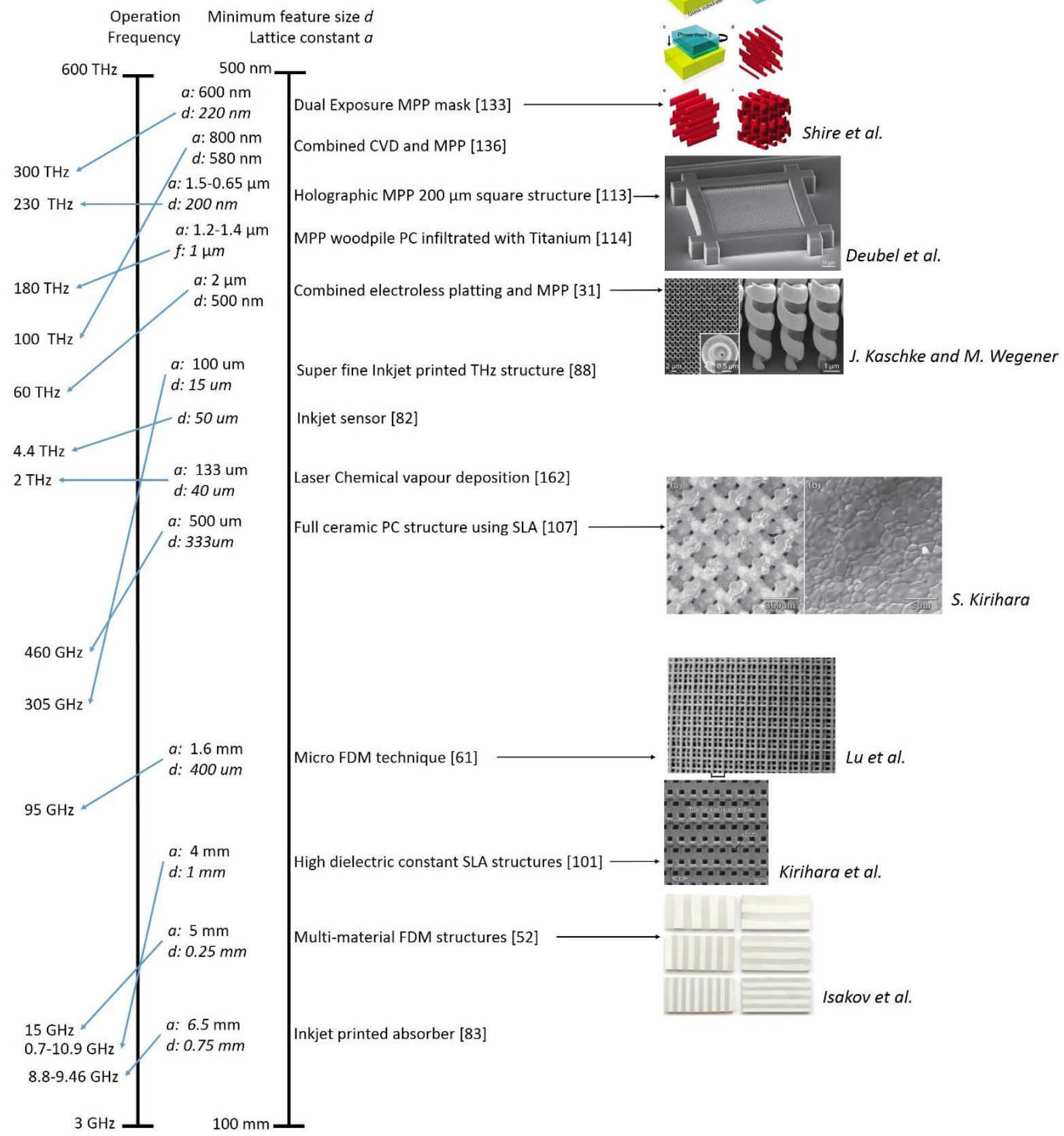

Figure 14 Graph of different structures fabricated using AM techniques with their feature sizes and their operating frequency. It is clear that the operation wavelength of the structures is usually smaller than their feature sizes.

\section{Acoustic Metamaterials (AMMs)}

\subsection{Introduction}

Acoustic metamaterials (AMM) are of interest due to applications in cloaking, imaging, and the fact that control of sound fields can be enhanced[166-170]. In noise and vibration control, enhanced sound absorption can also be achieved [171]. AMMs have been described with many 
different structures, all of which are designed to produce effects such as negative refraction, negative elastic moduli etc. A wide range of metamaterials has been proposed for using such effects to control acoustic wave propagation, where typically a base material is modified to contain holes, channels, resonators or scattering sites. The resulting material can have a negative bulk modulus (which in conventional materials would be expected to have a positive value). This can result in a negative acoustic refractive index and other exotic properties, many of which can only be manufactured using AM methods [172].

AMM structures can be considered to fall within three broad categories:

(i) Phononic crystals - These contain regular arrays of acoustic scattering and/or resonating sites within the material $[173,174]$. The creation of band gaps is possible in such structures, where a range of frequencies exist over which the transmission of acoustic energy across the material is not possible. This has many uses in acoustic absorption, filtering and isolation. In addition, such materials can exhibit a negative index behaviour, potentially allowing acoustic signals to be focused at particular frequencies at a resolution beyond the usual diffraction limit $[173,175,176]$. A subset of such structures is the creation of a plate containing regularsubstructures, rather than a three-dimensional crystal.

(ii) Resonant structures - Arrays of holes, channels or other embedded structures create a set of resonances. In channelled materials, enhanced sub-wavelength imaging resolution by the coupling of evanescent waves is possible $[7,177]$. They can also be used for acoustic filtering and sound control.

(iii) Space coiling - Extended curved or folded acoustic paths within a material can lead to negative elastic moduli and other interesting properties [178-180]. These sometimes contain elements that exhibit Fabry-Perot resonances, a subcategory of resonant structures to be discussed in section 3.3.

As in EM metamaterials, common to all AMM structures is the fact that functional structural components within the metamaterial tend to be much smaller than the wavelength of the acoustic signal. The ability of AM techniques to manufacture complicated shapes at small scales is thus an advantage in many cases. The choice of AM method often depends on the minimum build resolution required to realise the complex elements within the structure. A range of different methods have been compared recently in a study that compared three different polymer-based printing technologies to numerical models and a metal powder-based print technologies, where it was shown that surface roughness and accuracy of build had an effect on the acoustic performance[181].

The following sub-sections will consider the role of AM in the manufacture of different types of acoustic metamaterial. It is convenient to discuss the most appropriate AM method for each of the three main types of AMMs separately - each can be made through a variety of AM techniques. They are here considered as structure types to clarify each different mode of metamaterial behaviour. 


\subsection{Phononic crystals}

Phononic crystals are materials that originally were conceived as a regular array of acoustic scattering sites or inclusions. These sites typically have an acoustic impedance $(Z)$ that differs from the host medium, so that a significant amount of energy is reflected at each scattering location. This definition has now been broadened to include many types of regular structures containing features that rely on combinations of scattering and resonances. Several books have been written on their method of operation, to which the reader is referred $[18,182]$.

One of the primary results obtained with a phononic crystal is the existence of band gaps regions within which acoustic propagation is forbidden due to a combination of multiple scattering and local resonances. Thus, the design criteria are often defined with a view to creating these band gaps in the frequency range of interest, as these usually infer other properties related to high-resolution imaging.
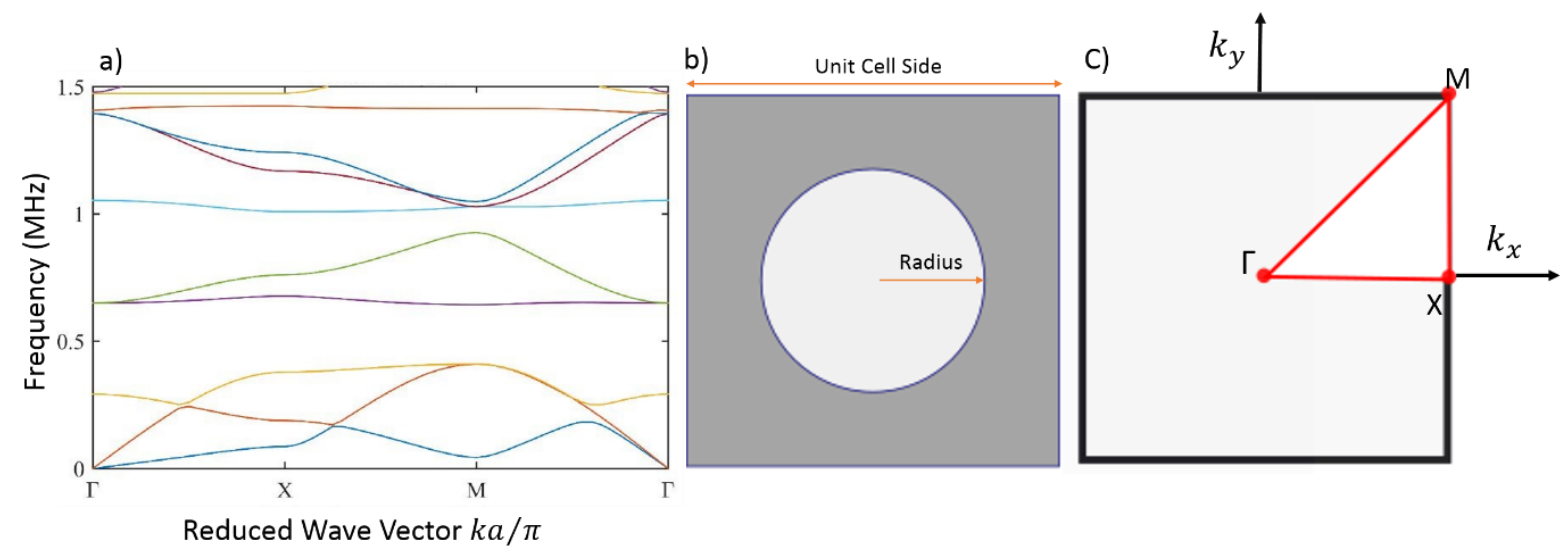

Figure 15 2D COMSOL simulation of the irreducible Brillouin zone of a periodic polymer structure with circular holes. The unit cell size of $1 \mathrm{~mm}$ and the hole dimension of $0.475 \mathrm{~mm}$ were chosen in this simulation. a) Plot showing the existence of bandgaps, $b$ ) the unit cell and c) irreducible Brillouin zone with marked directions.

The design process involves modelling the interaction of acoustic signals with the regular scattering structure and requires consideration of the properties of both the scattering sites and the host medium (sometimes referred to as the matrix). In addition to band gaps, it is of interest to construct structures that become dispersive, i.e. where the wavenumber in particular directions in the crystal is a function of frequency. It is this phenomenon that ultimately leads to the possibilities of enhanced imaging (greater intensity and subwavelength information), negative refraction (refraction in the opposite direction to standard which allows focusing), and other exotic phenomena, as detailed by the pioneering work of Page and co-workers $[173,174,183]$ and later reviewed by Lu et al. [184]. It is thus crucial to be able to predict which structures lead to the required exotic behaviour. This, in turn, leads to the requirements needed from any AM method in terms of build area and resolution. Prediction of behaviour can typically take place using either Finite Element (FE) modelling or multiple scattering theory.

Various types of phononic crystals have been designed and fabricated using non-AM methods, many of which consisted of solid spheres or rods, with or without coatings, embedded within 
a polymer or liquid substrate $[185,186]$, but this limits the size and complexity of structures that can be investigated and also how they might be applied. The issues that arise during the design and fabrication of such structures via AM methods have been described by Lucklum and Vellekoop [187]. There are various choices for the method of fabrication, depending on the frequency range of interest. At low frequencies, such as that needed for vibration control, Fused Deposition Modelling (FDM) can be used. An example is that of Matlack et al. [188], who created a rectangular polymer skeleton within which metallic cubes were placed. Their structure, which was designed for use at frequencies of up to $10 \mathrm{kHz}$, is shown in Figure 16. The cubes act as resonating elements, which act in addition to the periodicity to create wider band-gaps for vibration isolation. (Note that the metallic cubes were placed within the structure manually).
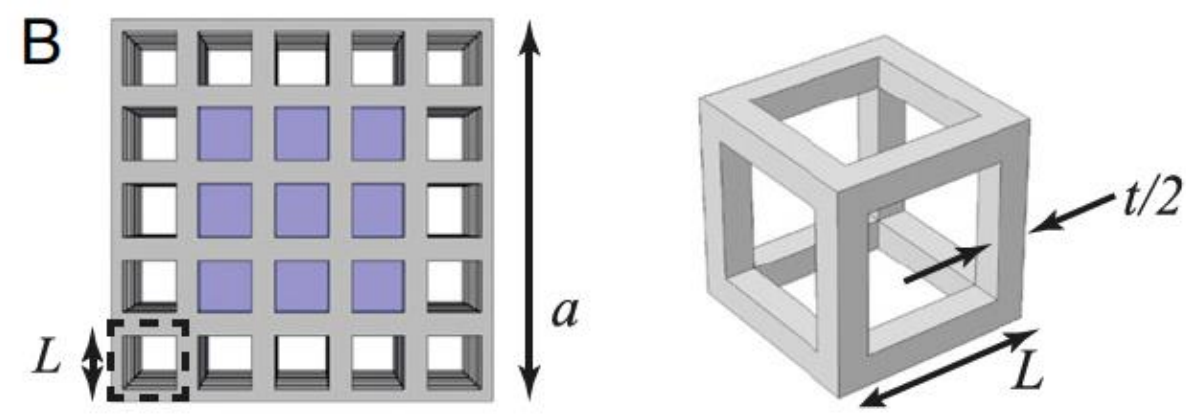

Figure 16: (a) Metamaterial structure built by Matlack et al. [188] using FDM, with metal cubes (shown in blue) inserted manually within the polymer grid. (b) The unit cell used in defining the grid structure.

Another approach is to use a face-centred cubic lattice of thin-walled hollow trusses [189] joined at a series of apexes by connectors, each of which could be made using AM processes; however, manual assembly is still required. More simple structures, more amenable to a single AM technique, would use one solid material only, such as a solid structure containing scattering air-filled voids, defined by a simple unit cell which is then repeated throughout the structure during the build. The aim in many of these metamaterials is the creation of a wide bandgap and the greater the acoustic impedance mismatch between the solid and air the better. Luckily, the acoustic impedance mismatch between a polymer or similar material and air is sufficiently large that very efficient scattering within the polymer matrix from the air-filled voids would occur. The choice of fabrication route depends upon the build resolution required. D'Alessandro et al. [190] chose Selective Laser Sintering (SLS) of Nylon to achieve a structure shown in Figure 17, together with the unit cell topology. The dimensions of this structure made it suitable for use at frequencies of up to $20 \mathrm{kHz}$. 


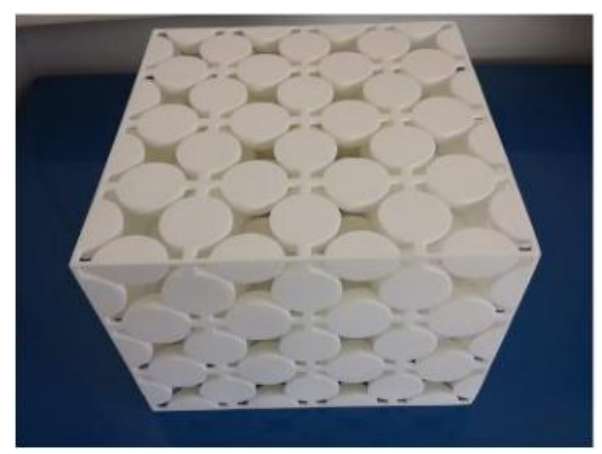

Figure 17: (a) Nylon phononic crystal metamaterial manufactured using SLS [190].

AM was also used by Zhang et al. [191] to create a structure whose properties were graded (i.e. varied smoothly) in the direction of sound travel. Although the exact AM method and photosensitive polymer were not defined, the frequency range examined was up to $6.3 \mathrm{kHz}$, so the cell sizes were the same order of magnitude to those of D'Alessandro [190]. It was demonstrated that grading the structure gave advantages in terms of bandgap quality, of interest to applications in sound and vibration isolation.

For use at higher frequencies, a higher build resolution is required. Lucklum and Vellekoop $[192,193]$ showed that for work in the ultrasonic range $(100 \mathrm{kHz}-2 \mathrm{MHz})$, a good fabrication approach would be Micro Stereolithography (MSL). This was used to create a regular array of air-filled voids, effectively with the shape of three intersecting cylindrical channels. Figure 18(a) shows an optical image of a fabricated structure, and Figure 18(b) illustrates the unit cell used in the design. The build gave reproducible features at the $50 \mu \mathrm{m}$ level, which was sufficient for defining structural features that were a small fraction of the incoming wavelength (at frequencies of up to $2 \mathrm{MHz}$ or so). Simulations and experiments were in agreement, with a bandgap appearing over a $0.4-1 \mathrm{MHz}$ frequency range, due primarily to scattering from the regularly-spaced air-filled voids. It should also be noted that Lai and Zhang [194] have predicted that using inclusions with different radii could introduce improvements in performance, although this was not confirmed experimentally. MSL was also used by Laureti et al. [195] to generate a PnC having regularly-spaced steel spheres embedded within an acrylic matrix. This was achieved by creating the first layer of acrylic containing spherical depressions into which spheres were placed manually; the next acrylic layer was printed on top and the process repeated. Experiments in a water tank at frequencies of up to $1 \mathrm{MHz}$ showed the presence of focusing effects, which would also be expected from such structures. With the band gaps present in the transmission of these structures, negative refraction may occur, providing enhanced focussing.
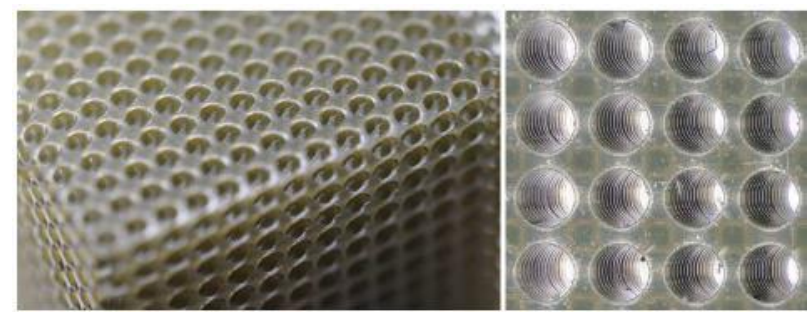

(a)

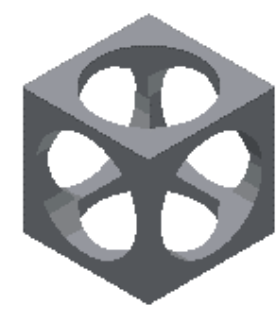

(b) 
Figure 18: (a) Phononic crystal metamaterial structure printed using MSL by Lucklum and Vellekoop [192]. (b) The unit cell used in defining the structure.

An alternative technique for fabricating complicated regular structures is robocasting [196], which can be used to create scaffold structures from a ceramic material. This was the approach used by Kruisová et al. [197], where various hexagonal and tetragonal structures fabricated from silicon carbides $(\mathrm{SiC})$ were designed, modelled and tested. The geometries, unit cells and FE modelling meshes are shown in Figure 19. Characteristic length-scales (for unit cells) were 0.2-0.7 mm. The authors found that band gaps were created in the 5-10 $\mathrm{MHz}$ frequency range, a region not extensively explored in the metamaterial literature but of importance in biomedical diagnostic imaging. Note that earlier work by the same authors had explored regular tetragonal lattices, and characterised the build process and the resulting band structure [198]. It was notable that very low damping was observed, this being a useful attribute in many practical applications.

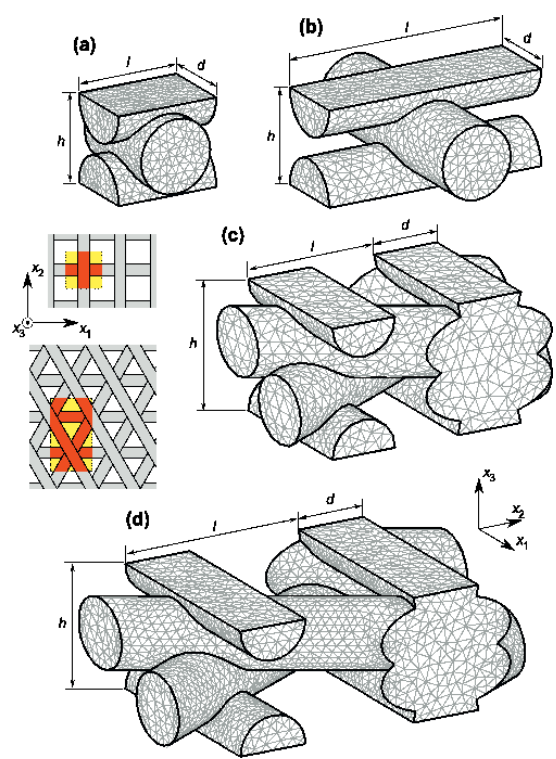

Figure 19: Geometries and FEM meshes used by Kruisová et al. [197] for calculations of the band structure of their scaffold structures: (a) SS structure; (b) LS structure; (c) SH structure; (d) LH structure. Schematic sketches on the left show the placements of the unit cells in tetragonal $(a, b)$ and hexagonal $(c, d)$ structures in the $x_{1}-x_{2}$ plane.

Another AM method that has been used to investigate phonic crystals is Electron Beam Melting (EBM). The existence of band gaps in strut-based (or "lattice") metamaterials had been predicted [199], and EBM allows such structures to be investigated experimentally, as detailed by Wormser et al. [200,201]. One design [200] consisted of Ti-6Al-4V curved struts, typically 
$<0.5 \mathrm{~mm}$ in diameter, joined together at nodes at a typical distance of $5 \mathrm{~mm}$. In both measurements and simulations, a partial bandgap (i.e. not appearing in all directions) was observed within a frequency range of $75-110 \mathrm{kHz}$. The design was further evolved into one containing cuboid masses at the nodes [201,202], again joined by either straight or curved struts, with the variable "A" indicating the amplitude of the deviation from a non-curved strut. An example of the unit cell structures used for fabrication is shown in Figure 20, for four values of "A" (zero meaning a straight strut). Note the main differences to the design of Matlack et al. [188] shown earlier in Figure 16 - now the material is made in one AM process, using a single material. The result was the creation of multiple band gaps whose width at frequencies of up to $150 \mathrm{kHz}$ exceeded those of the simulations, for all the four designs shown in Figure 20, with the bandgaps decreasing in centre frequency with increasing " $\mathrm{A}$ ". The authors attribute this to the cuboid masses acting as inertial elements and interacting with the flexible struts.

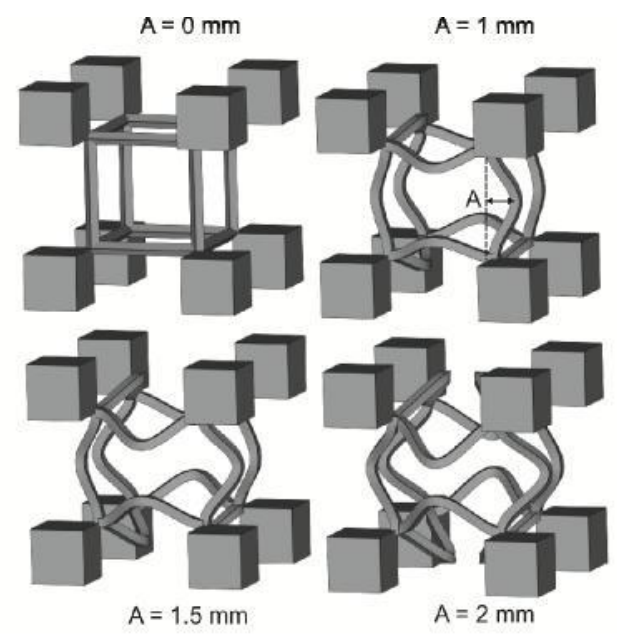

Figure 20: The four unit-cells used by Wormser et al. [201] to fabricate a PnC via EBM having a range of bandgap properties.

Another type of strut-based acoustic metamaterial is that created by the so-called pentamode configuration. Here, the struts are of varying thickness in the form of a double cone geometry, thicker at their centre and joining together at a point. Selective laser melting (SLM) was used to create the structure from Ti-6Al-4V metal powder [203]. This approach was compared to polymeric structures manufactured using Direct Laser Writing (DLW) optical lithography, where lower elastic moduli were observed. Such structures have also been reported by other authors (e.g. [202]) as being suitable for AM manufacture in polymers, although the build process is complicated due to the fragile nature of the struts. The property of interest acoustically was the ratio of the static bulk to shear moduli, which was found to be approximately $10^{3}$.

An interesting addition to the research on lattice metamaterials is where the structure can be deformed using external fields. This has been demonstrated by Yu et al. [204], where external magnetic fields were used to alter the geometry of a lattice metamaterial that had been created using stereolithography-based AM methods. 
It is interesting to note that two-dimensional metamaterial plates can also be used to create acoustic band-gaps for waves travelling along the plate as Lamb waves, one example being the positioning of a regular array of masses on one surface [205,206]. In addition, the use of resonant rod-like structures can achieve band gaps for surface waves [207]. AM techniques such as FDM are suitable for the manufacture of such structures at low frequencies. Also, it has been shown that additional holes in an FDM-manufactured plate, fitted with regularlyspaced cylindrical masses, can create a trampoline-like effect, which helps to create more numerous and wider band-gaps [208].

\subsection{Fabry-Perot and space coiling (labyrinthine) metamaterials}

Fabry-Perot structures contain arrays of cavities of sub-wavelength diameter, the resonance within which leads to the special properties of the resultant metamaterial. Many reports in the literature are for structures produced using conventional manufacturing methods at a larger scale for use in the air at low frequencies. However, some authors have used AM methods. Laureti et al. [195] used FDM to manufacture a sample containing 100 channels, in front of which was placed an L-shaped aperture of sub-wavelength dimensions (Figure 21). It was shown that the "L" shape could be retrieved on the far side of the metamaterial in the air at $11.48 \mathrm{kHz}$, at a resolution well below the conventional diffraction limit at the chosen frequency.

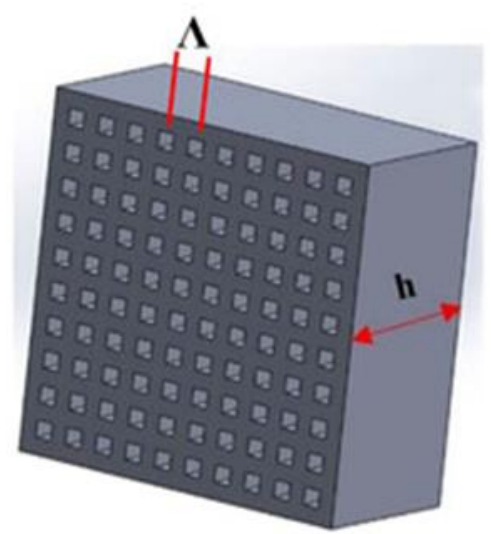

Figure 21: The Fabry-Perot metamaterial used in the air by Laureti et al. with $h=14.8 \mathrm{~mm}$ and $\Lambda=2.96 \mathrm{~mm}[195]$.

An alternative design was developed for its sound absorption properties [209]. This used concentric cylinders of various lengths $\left(h_{1}, h_{2}\right.$ etc.) which were closed at one end and segmented radially, as shown in Figure 22. Here, the quarter-wave resonances of individual cylinders were tuned to the appropriate frequencies to both broaden and tailor the acoustic absorption response to the design values. In addition, other authors have used cylindrical Helmholtz resonators of approximately $0.5 \mathrm{~mm}$ diameter to attenuate sound in the audible range. Stereolithography was 
used to allow such small structures to be fabricated, with the Helmholtz resonators used to create stop bands at specific frequencies [210].

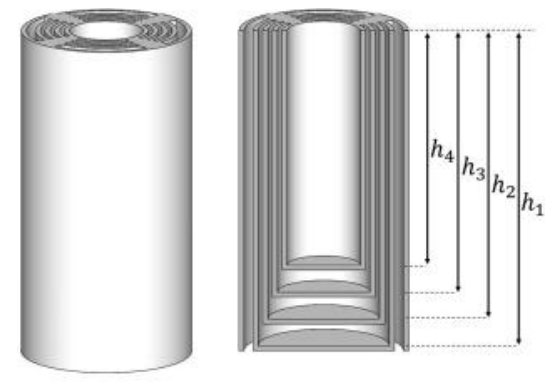

Figure 22: Resonant structure using concentric cylinders of different lengths for sound absorption in the air [209].

Space-coiled (labyrinthine) acoustic metamaterials are those where the cavity containing the acoustic field takes a tortuous path. AM methods are one of the few ways in which most of these materials can be manufactured. In general, they consist of extended, often folded paths through an object, whereby changes in amplitude and phase leads to exotic acoustic properties at the output [179]. One example is in the control of sound absorption [211]. Another example is wavefront shaping and focusing effects, where it has been shown for a metasurface how the use of a horn geometry can lead to advantages, including simultaneous phase and amplitude modulation [212]. Note that Fabry-Perot resonances can occur in the folded transmission channels. This effect has been used, for example, in the creation of a Fresnel lens, fabricated via AM methods, for use in the air at frequencies of up to $10 \mathrm{kHz}$ [21]. In other work, FDM AM was used in the air at $2.3-3.7 \mathrm{kHz}$ to create changes in phase across a lens to achieve focusing [213]. The design of the lens and the manufactured FDM object are shown in Figure 23(a) and (b) respectively.

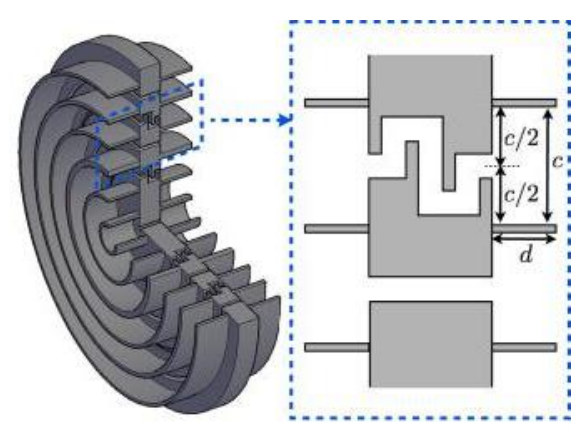

(a)

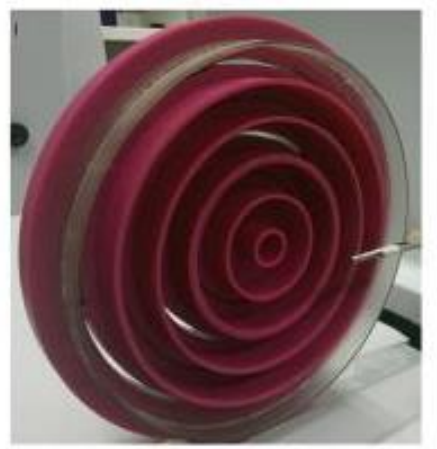

(b)

Figure 23: (a) Design of a Fresnel lens using space-coiling techniques, and (b) the lens manufactured using Fused Filament Deposition AM (adapted from [21]).

Space coiling was also used within a novel approach which used metamaterial "bricks", fabricated by 3D-printing [214]. Each brick could be designed to have a different phase delay, for example, which could then be used to create phenomena such as beam steering. The device was also used to demonstrate acoustic levitation. 
With the correct design, such metamaterials can exhibit a negative refractive index, leading to possibilities such as high-resolution imaging. If local resonances are avoided, space coiling can create doubly-negative structures using features that are sub-wavelength in dimensions, as demonstrated by Maurya et al. [215] using FDM fabrication with ABS polymer at frequencies of up to $4 \mathrm{kHz}$ in the air. Phase gradients were also created by Xie et al. [216,217] to demonstrate extraordinary beam steering and negative refraction at similar frequencies, using FDM fabrication methods. These researchers have also published a method for the systematic design of space-coiling structures suitable for AM fabrication for operation in the air [218].

AM methods lend themselves to different geometries. For example, laser-based stereolithography was used to create a helical metamaterial within which acoustic propagation occurred in the solid, rather than in a channel within the solid [219]. A similar concept was used by Ding et al. [220] to create an impedance matching layer with a graded-index. FDM and similar methods have been used to create labyrinth-type surfaces for the control of reflected waves [221], and to create an ultrathin metasurface [222], both for acoustic signals in the air. More exotic shapes and processes have been reported. For example, a chiral material (with properties like negative refraction which are analogous to those seen in optics) has been designed and printed [223], and a 2D device for rotating the wavefront of an acoustic signal has also been reported [224].

\subsection{Isotropic three-dimensional metamaterials}

The possibilities created by AM techniques for manufacturing 3D metamaterials are wellillustrated by the ability to print spherical objects with metamaterial properties. For example, a 3D space-coiling sphere has been produced by Fu et al. using AM techniques [20] and is shown in Figure 24(a). This exhibited both monopolar and dipolar resonances due to the space-coiling structure within it. This object blocked sound wave propagation at frequencies between these two resonances but also had negative values of effective mass density and effective bulk modulus around the dipolar and monopolar resonances respectively. This leads to possibilities in areas such as acoustic cloaking, where spheres of different sizes could be used to extend the range of frequencies in a particular application.

A different mechanism was used by Xie et al. [225], based on a cubic structure. Here, the aim was to produce a structure that could be fabricated using an SLA printer, in which the effective properties such as refractive index could be controlled across the object. In the example shown in Figure 24(b), the object was made in two hemispheres that were then joined together. The resulting acoustic lens, the operation of which was based on an earlier work using metal rods to create the metamaterial structure [226], is known as a Luneburg lens, and represents a type of graded-index acoustic (GRIN) lens. 

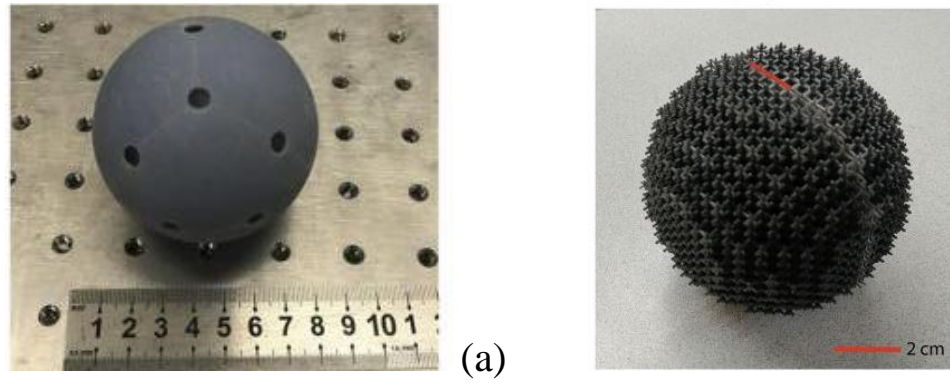

(b)

Figure 24: Spherical acoustic metamaterial structures using (a) space coiling (taken from [20]) and (b) a metamaterial containing a cubic structure (taken from[225]). 


\section{Mechanical Metamaterials}

\subsection{Introduction}

Mechanical metamaterials are rationally designed materials with certain mechanical properties which are defined by their geometry, e.g. their micro/nano lattice architecture, rather than their composition [34,36,227]. Mechanical metamaterials can be designed and tuned to deliver a range of mechanical properties [228]. These include elastic modulus, strength, Poisson's ratio, recoverability, energy absorption, thermal expansion etc. Different types of mechanical metamaterials and their properties can be found in the excellent review papers on this subject [34-38] [228]. Auxetic [22,23,229-237] and pentamode [238] metamaterials, ultra-light metallic/ceramic micro-/nano-lattices [239], carbon nano-tube based structures [240] and different classes of flexible metamaterials [241] are examples of mechanical metamaterials that have been studied extensively.

As stated above, auxetic materials exhibit a negative Poisson's ratio. Based on their microstructure design, auxetic materials have been classified into three main groups including re-entrant (e.g. a bow-tie structure), chiral, and rotating (semi-) rigid structures [242]. Enhanced resistance properties against damage, improved indentation resistance, better acoustic/vibration properties and high energy absorption have been reported for auxetic materials [36,242].

Pentamode materials, also called meta-fluids, have a very large value of bulk modulus compared to their shear modulus causing their volume not to change under deformation (i.e. having a Poisson's ratio of 0.5 ). A frequently studied design of pentamode microstructures includes a specific arrangement of conical beams in a diamond-type lattice structure [202,238,243]. Increasing the density of this lattice design has minimal effect on the stiffness as opposed to Ashby plot [244] which suggests a power low relationship between density and elastic modulus.

Introducing random porosity to a solid material disproportionally depredates the mechanical properties. An example is the stiffness or elastic modulus $(E)$ of stochastic materials such as aerogel and foam which according to power-law is poorly scaled with density $(\rho)$ as $E \sim \rho^{3}$ [245]. Micro/nano-structure of ultralight ultrastiff metamaterials [239,245] are rationally designed to deliver close to linear scaling of stiffness/strength and density. This includes the use of hollow beams in the microstructure of a stretch-dominated lattice structure [228].

The ability to fabricate high-resolution 3D structures directly from a CAD file and the capability to individually tailor the unit cells of design makes AM technologies a viable option for fabrication of mechanical metamaterials. Below, the most common additive manufacturing processes for the fabrication of most common types of mechanical metamaterials are reviewed.

\subsection{Vat polymerization}

Vat polymerization techniques have been the most commonly used AM techniques for the fabrication of mechanical metamaterials. These techniques have been extensively used for the fabrication of extremal metamaterials such as auxetic and pentamode structures [246,247]. 
Buckmann et al. demonstrated auxetic metamaterial structures with sub-micron unit cells which were fabricated using MPP [248]. For the first time a structure with the overall size of $30 \mu \mathrm{m} \times 30 \mu \mathrm{m} \times 30 \mu \mathrm{m}$, composed of 108 bow-tie elements was fabricated using the MPP system and a Poisson's ratio of -0.13 was achieved [248]. Macroscopic and microscopic polymer auxetic structures fabricated using material jetting (objet30) and dip-in mode of MPP (Nanoscribe GmbH, Germany) demonstrated maximum Poisson's ratio of -0.8 [233]. Microstructured polymer pentamode metamaterials with bulk to the shear ratio as large as 1000 were fabricated using DLW process [243]. Laminate heterostructures with enhanced flexibility processed through optical laser lithography (Nanoscribe GmbH, Photonic Professional GT) were introduced as modified pentamode metamaterials [249].

The unfeelability cloak is the mechanical equivalent of an invisibility cloak for optical metamaterials [238,243,246]. Using a commercial MPP system, Buckmann et al. ( [246], see Figure 25) and Kadic et al.[249] fabricated a pentamode metamaterial that can elastically hide objects. The unfeelability cloak was built using the dip-in mode of the MPP setup. Using a refractive indexed matched photoresist, it is possible to push the objective lens inside the photoresist and fabricate structures which are taller than the working distance of the objective lens. The commercial MPP setup uses a Galvo mirror system to fabricate structures with high speed $\left(5 \mathrm{~cm} \mathrm{~s}^{-1}\right)$ [246]. One of the shortcomings of the MPP system is its limited build volume. To rectify this issue it is possible to divide a design into a number of areas and fabricate larger structures in consecutive attempts. The piezoelectric stage in the commercial MPP system enables the user to stitch each part to the previous parts. Pentamode metamaterial structures with bulk modulus to shear modulus ratio in the range of $>10^{3}$ was achieved using polymer microstructures fabricated using the dip-in mode [243].

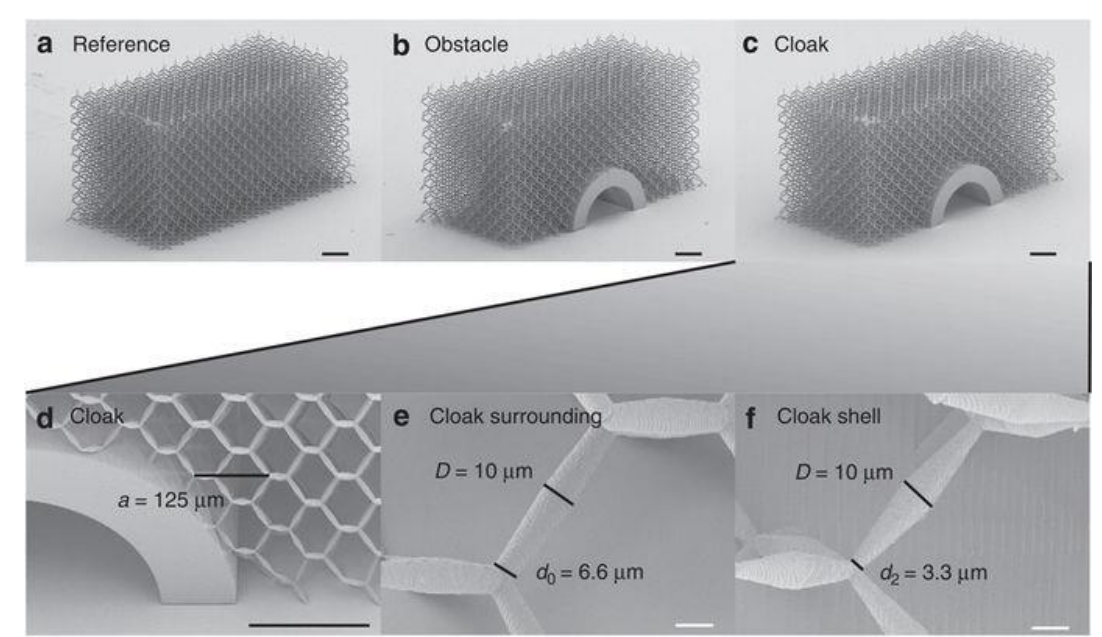

Figure 25 Unfeelability cloak fabricated to elastically hide the solid cylinder placed underneath the structure [246].

Mechanical metamaterials with other functionalities different than extremal material have also been processed through vat polymerization techniques. Frenzel et al. [223] designed and fabricated mechanical metamaterials which were able to twist under compression (Figure 26). 
These metamaterials were fabricated using 3D laser micro printing, and they could deliver twists per axial strain exceeding $2 \%$.

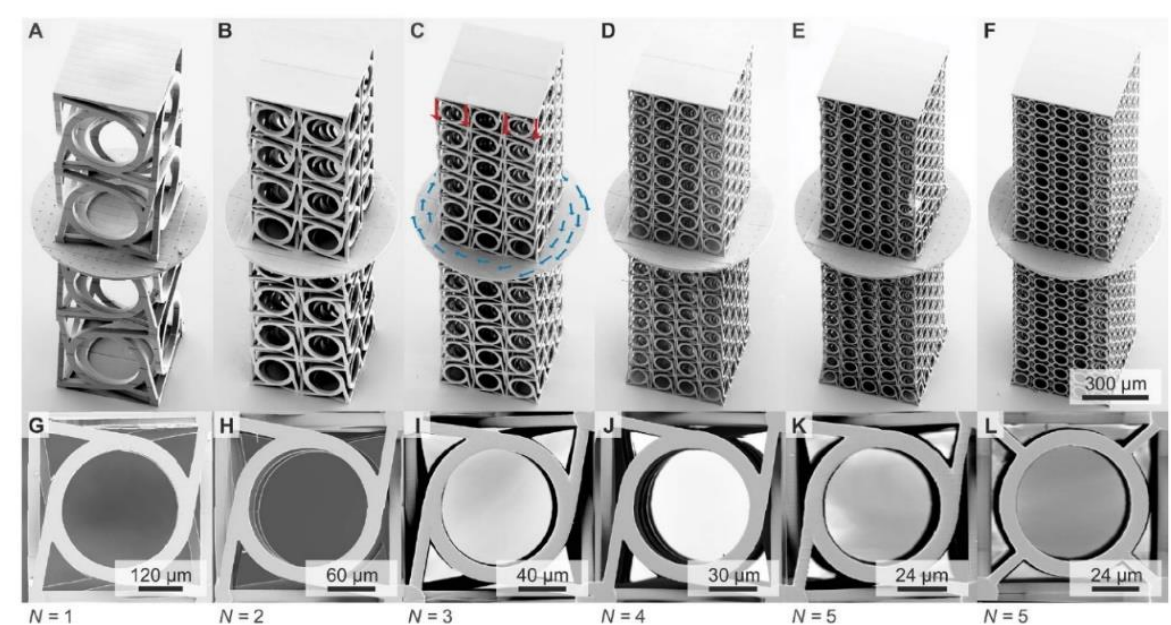

Figure 26 Mechanical metamaterials with a twist [223]

Mechanical metamaterials with tunable negative thermal expansion have been fabricated using multimaterial projection MSL (Figure 27) [250]. It is possible to tune the negative thermal expansion in a dual material unit cell by varying the thermal expansion coefficient differences and the topology of the unit cell. Zero-thermal expansion coefficient is also possible to be realized using the same strategy by offsetting the positive and negative effects within the unit cell [250].

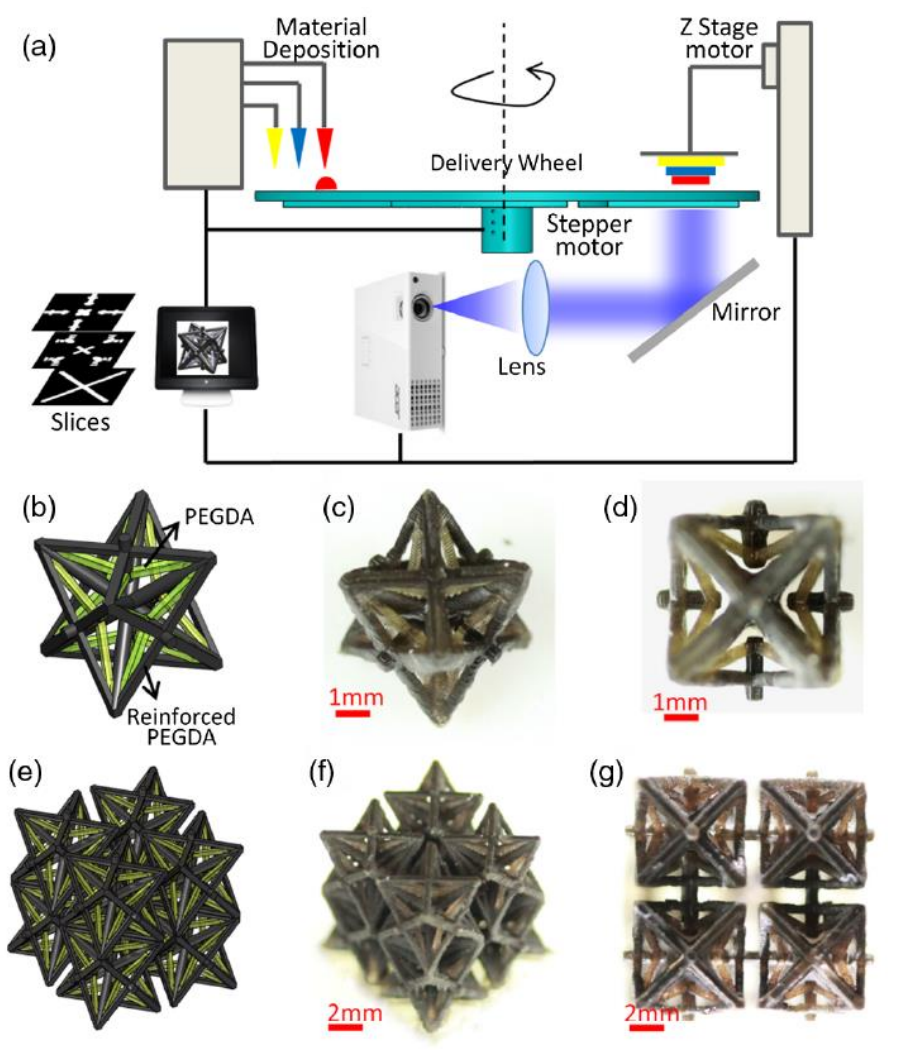


Figure 27 Mechanical metamaterials with tunable negative thermal expansion fabricated using multi-material projection MSL [250].

Macroscopic octet-truss lattice structures processed through SLA have been investigated for recoverability and energy absorption [251]. The energy absorption efficiency of the lattice structures made from green resin (with mechanical properties similar to ABS) and grey resin (with mechanical properties similar to PMMA) was nearly $11 \%$ higher than Duocel® aluminium foam with a recoverability capability of strains up to $70 \%$ [251].

The self-propagating photopolymer waveguide technique is a vat polymerization technique that uses a collimated UV light and a polymer mask to fabricate open cellular structures (see Figure 28). This technique has the potential to reach mass-manufacturing of micro-lattice structures in large scale without affecting the lattice symmetry and diameter. It is a promising method for fabrication of practical mechanical metamaterials for real-life applications [239,252].

Currently, no AM process offers fabrication of ceramic or metal nanolattices at a high resolution [228]. To overcome this limitation, hybrid fabrication techniques have been used in which a vat polymerization process is initially employed to fabricate a polymer lattice, followed by post-processing techniques to fabricate metal/ceramic lattice from polymer lattice. Jacobsen et al. [253] used a self-propagating photopolymer waveguide technique to fabricate polymer micro-lattices. Glassy carbon micro-lattice structures were then fabricated using pyrolysis of the polymeric micro-lattices. Eckel et al. [254] used pyrolysis process to convert polymer micro-lattices manufactured by SPPW to ceramic micro-lattices. Glassy carbon nanolattices with strength close to the theoretical values and an improved stiffness to density ratio were manufactured by pyrolysis of polymer nanolattices 3D-printed through DLW (Figure 28) [255].

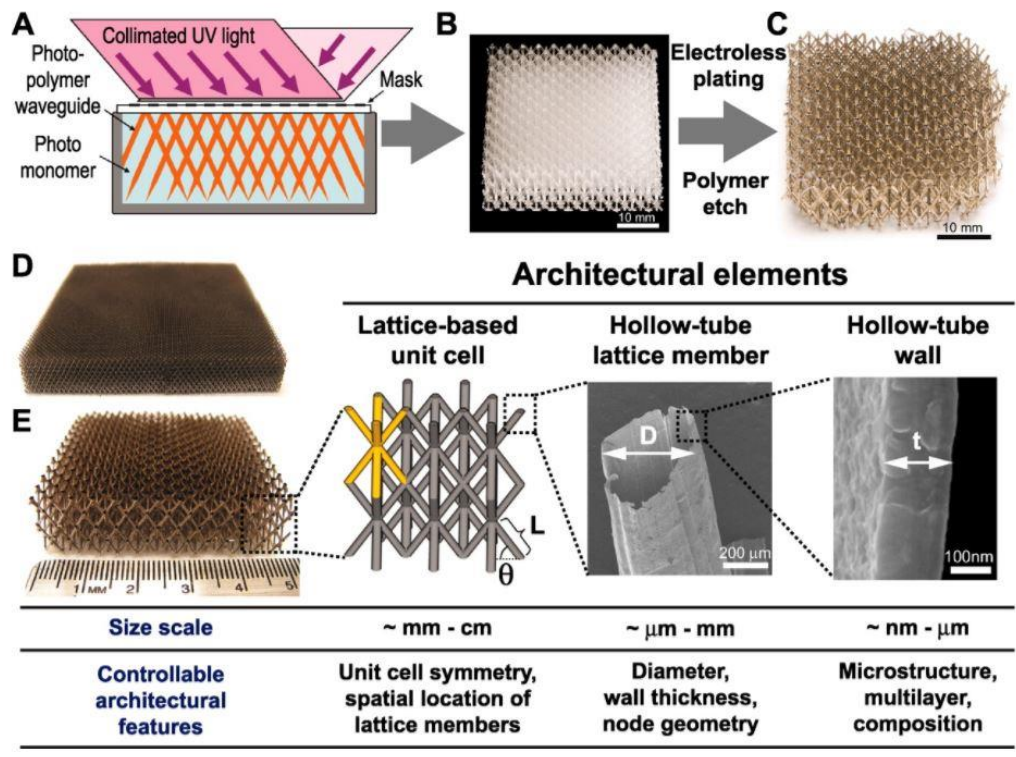


Figure 28: Structures fabricated using the self-propagation polymer waveguide technique with feature sizes spread over three orders of magnitude. (A) the basic diagram of the fabrication technique; (B) Polymer template; (C) Ni-P micro-lattice structure fabricated using electroless plating of the polymer template and etch removal of the polymer template. (D-E) two fabricated structures with a breakdown of their constructing elements [239].

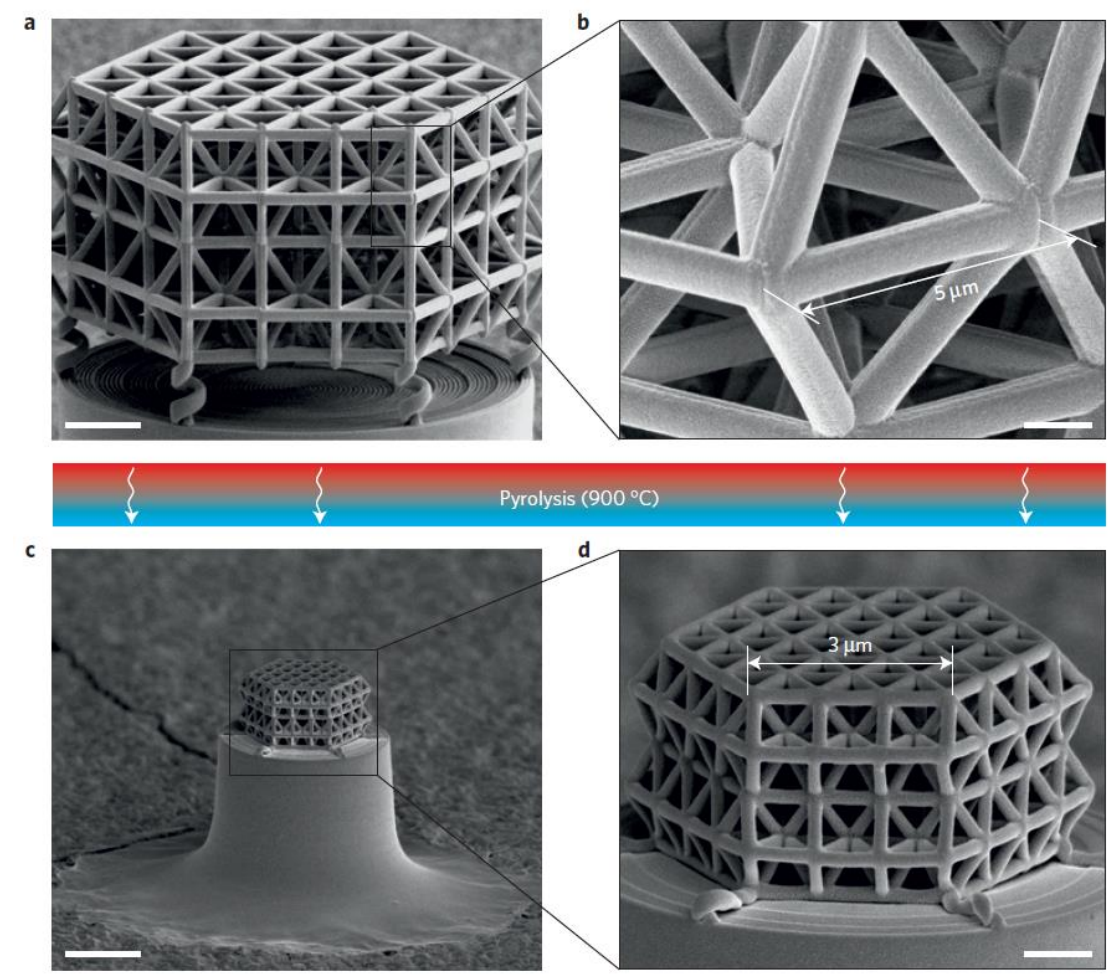

Figure 28: Glassy carbon nanolattices created by pyrolysis of 3D-printed polymeric microlattices [255].

Ultralight metallic micro-lattices consisting of hollow tube strut members have been fabricated using the Self-Propagating Photopolymer Waveguide (SPPW) technique to firstly manufacture the base polymer lattice, followed by electroless nickel plating and subsequent etching [239,256,257]. In [239] (

Figure ), the Young's modulus $(E)$ of the ultralight micro-lattices were observed to be proportional to $\bar{\rho}^{2}$ where relative density $\bar{\rho}$ is defined as the ratio of the apparent density $\rho$ to the base material density $\rho_{s}$. Hollow nickel microlattices of densities $\bar{\rho}<0.1 \%$ showed nearly full removability for compressive strains of up to more than $50 \%$, while micro-lattices of higher densities had fully plastic behaviour under compression [256]. The same technique was implemented for the fabrication of nickel hollow Triply-Periodic Minimal Surface (TPMS) micro-lattices [258]. Ultralight ultra-stiff stretch-dominated metallic micro-lattices with a resolution of nearly $5 \mu \mathrm{m}$ were fabricated using Projection micro-stereolithography technique combined with electroless nickel plating and subsequent thermal decomposition. Metalpolymer composite micro-lattices were fabricated by electroless plating of $\mathrm{NiB}$ on polymer scaffolds printed through DLW process [259]. Mechanical properties of the composite microlattices were highly dependent on the layer thickness of NiB. Ultra-strong copper micro-lattices were fabricated by DLW of polymer micro-lattices, electroplating on the lattice pattern and 
removal of the polymer [260]. The copper micro-lattices of cell size $6 \mu \mathrm{m}$ and density $\bar{\rho}>0.5$ was observed to be 1.8 times higher than the monotonic bulk copper. The reason for the enhanced stiffness of the copper micro lattices were attributed to the size effects associated with single-crystalline regions in the lattice beams. Hollow gold octahedral nanolattices [261] were fabricated by two-photon lithography of a polymer nanolattice which is then sputtered with columnar grained gold. The polymer scaffold was then subjected to focused ion beam and was removed by oxygen plasma etching. The yield strength of the Au nanolattices was increased by a factor of two by tuning the wall thickness of the nanolattices. This was in contrast to the classical mechanics of cellular solids which predicts constant strength and stiffness for all samples investigated in that study, highlighting the material size effects.

Ceramics benefit from high values of stiffness/strength to mass ratio. However, their brittleness limits their use as structural materials. Vat polymerization based techniques have been used to fabricate recoverable ceramic nanolattices [239,262,263]. Meza et al. [262] fabricated lightweight, stiff and recoverable hollow-tube nanolattices from Alumina with the ability to recover their original shape after compression up to more than $50 \%$ strain (Figure 29). The method used for the fabrication of the ceramic nanolattices included 3D printing of a polymer scaffold using DLW, deposition of a thin alumina film on the polymer scaffold using Atomic Layer Deposition (ALD), removing the outermost wall of the coated nanostructure using focused ion beam milling (FIB), followed by etching of the internal polymer. Similarly, hollow tube ceramic (alumina) micro-lattices were fabricated using MSL, ALD and thermal decomposition [250] (Figure 30). Unit cells scaling from 10 to $500 \mu \mathrm{m}$ have been fabricated using this technique. These ultralight ultra-stiff lattice structures demonstrated the desired relationship of Young's modules $(E) \sim \rho$ regardless of the material used.
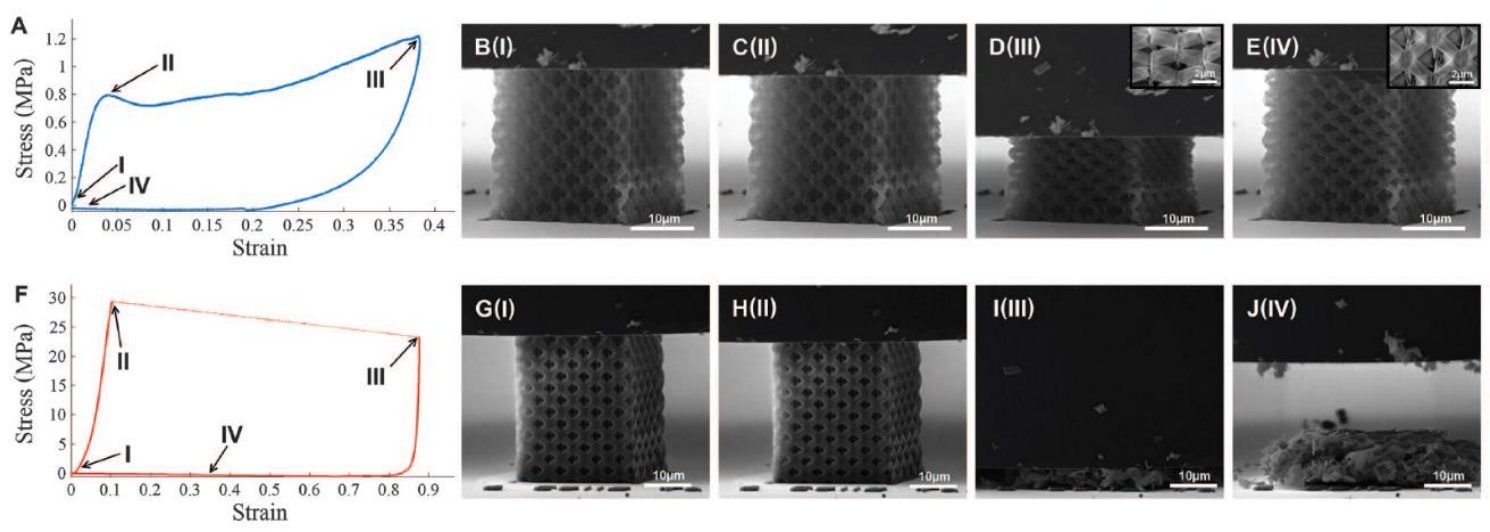

Figure 29: Compression experiments on thin-walled and thick-walled ceramic nanolattices. (A to E) - mechanical data, compression and recovery of a thin-walled nanolattice. $(F$ to $J)$ mechanical data, compression and brittle failure of a thick-walled nanolattice. [262] 

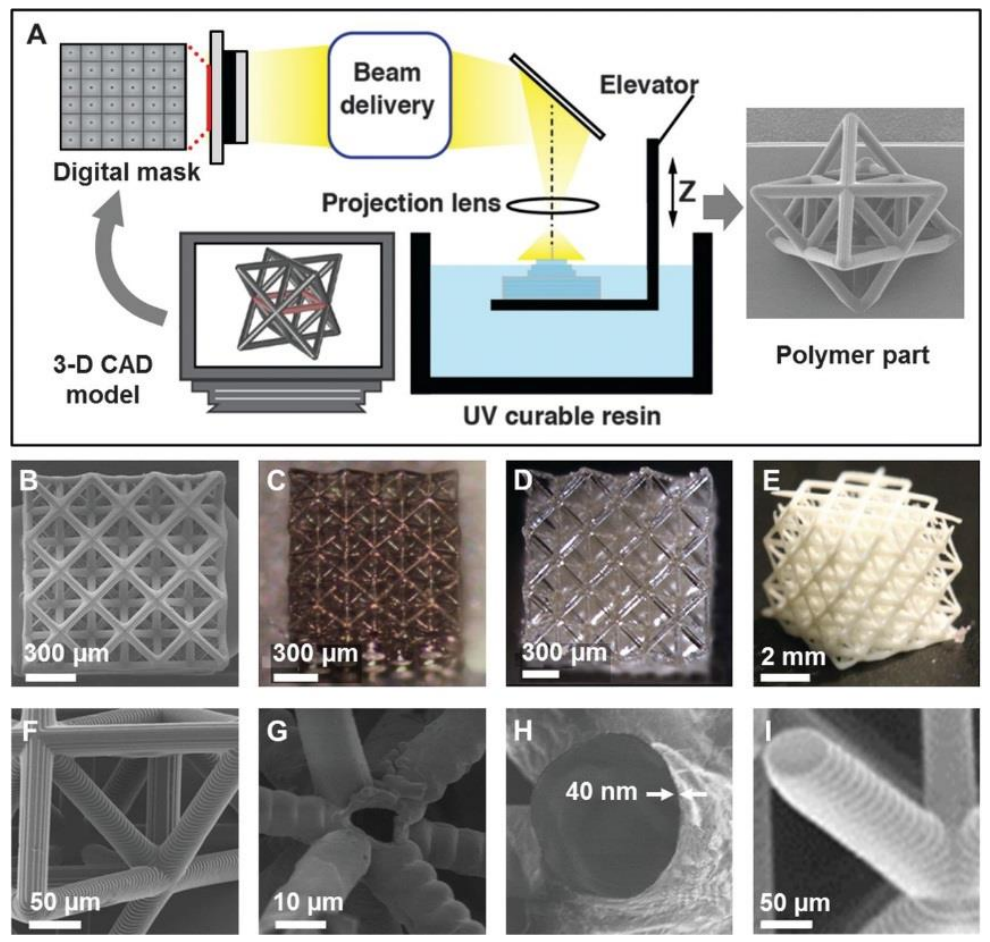

Figure 30: (A) Diagram of the SLA system used for fabrication of the lattice structure. (B) to (E) Octet-truss micro-lattice structures fabricated using SLA with different structures. $(F)$ to (I) magnified view of the structures in B to $E$ [245].

Hybrid fabrication techniques based on vat polymerization have been used for the fabrication of hierarchical hollow tube metamaterials [264-266]. Hierarchical nanolattices in the polymer, polymer-ceramic composite and ceramic (alumina) were fabricated implementing DLW, ALD and polymer etching [264,266]. The tensile yield strength of titanium nitride hollow tube nanolattices (Figure 31) ranging from $10 \mathrm{~nm}$ to $100 \mu \mathrm{m}$ was observed to be 1-2 orders of magnitude greater than that of bulk material which is likely due to the material size effects [266]. In a comparison made between first-order, second-order and third-order hierarchical nanolattices [264]. It was observed that second-order hierarchical nanolattices constructed from hollow tube ceramic struts benefitted from a high level of recoverability and close to linear scaling of strength and stiffness with relative density, while additional levels of hierarchy beyond a second order did not improve the mechanical properties (see Figure 32). Multiscale hierarchical metallic nanolattices have also been studied [265]. Within the structural hierarchy, a successive reduction of feature size from the macroscale to the nanoscale with an approximate factor of 10 between each hierarchy was implemented (Figure 33). To fabricate the hierarchical metallic nanolattices, a large area projection micro-stereolithography process was used in conjunction with electroless nickel plating and subsequent chemical etching of the polymer [265]. 


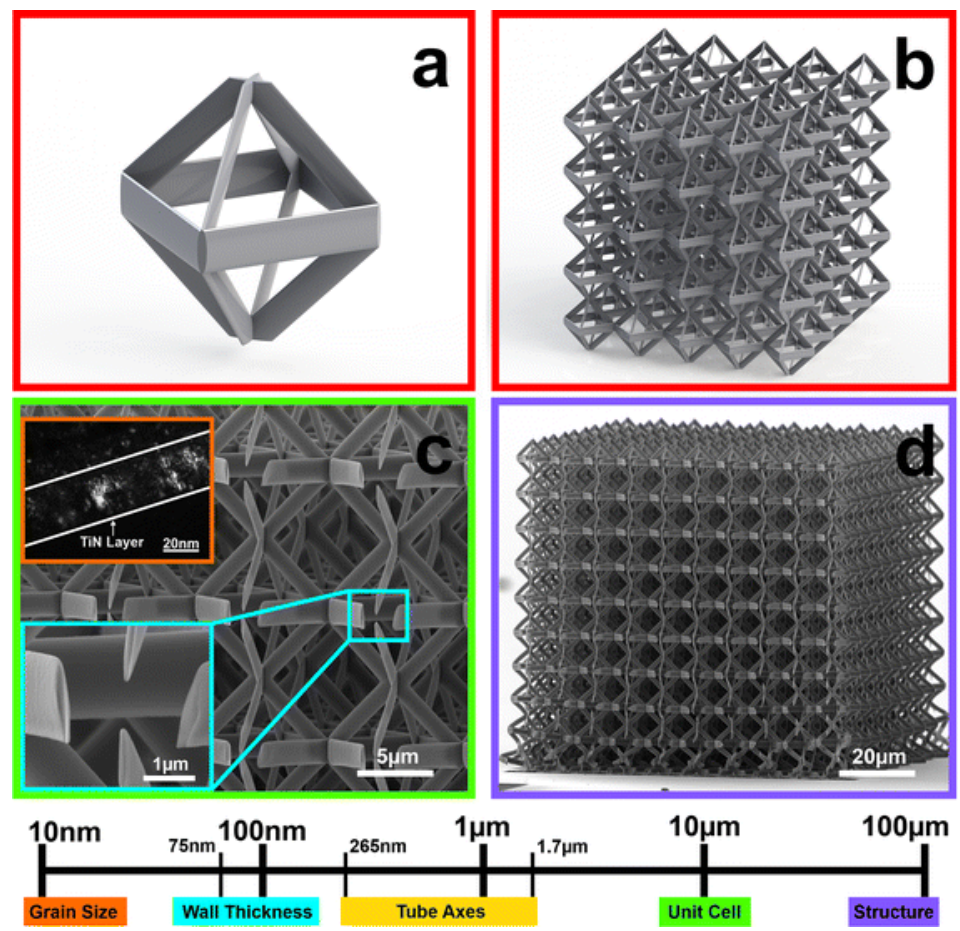

Figure 31: Nanolattice design and hierarchy. (a) A 3D design of a single unit cell of the structure. (b) The 3D design of the full nano lattice structure. (c) SEM image of a section of the fabricated structure. The top-left inset shows the dark field TEM image of the structure which shows the nano-sized grains and the bottom left inset shows TiN hollow tubes [266].
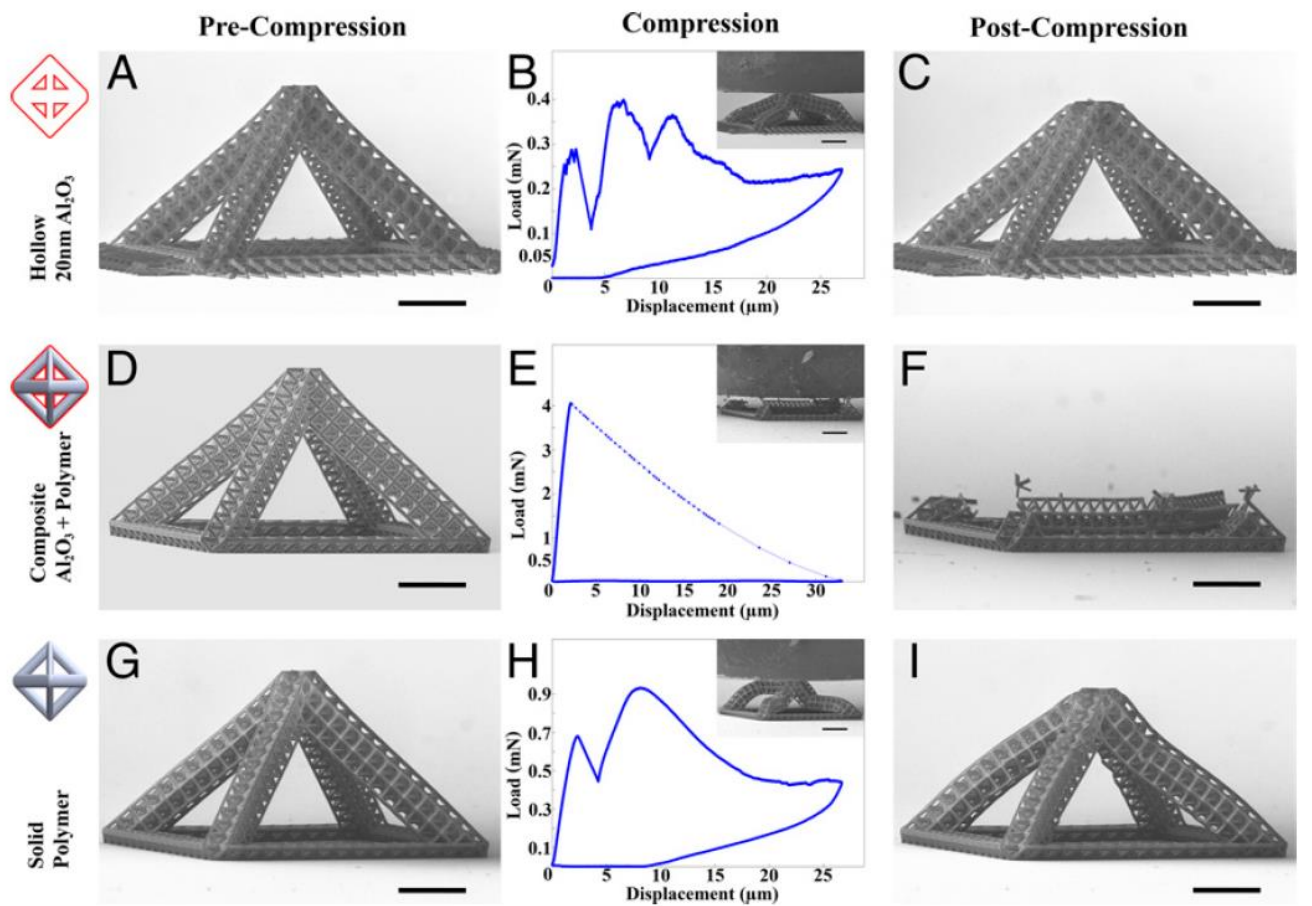

Figure 32 Comparison of compression behaviour of hollow ceramic $(A-C)$, ceramic-polymer composite $(D-F)$ and solid polymer $(G-I)$ second-order half-cell hierarchical nanolattices [264]. 


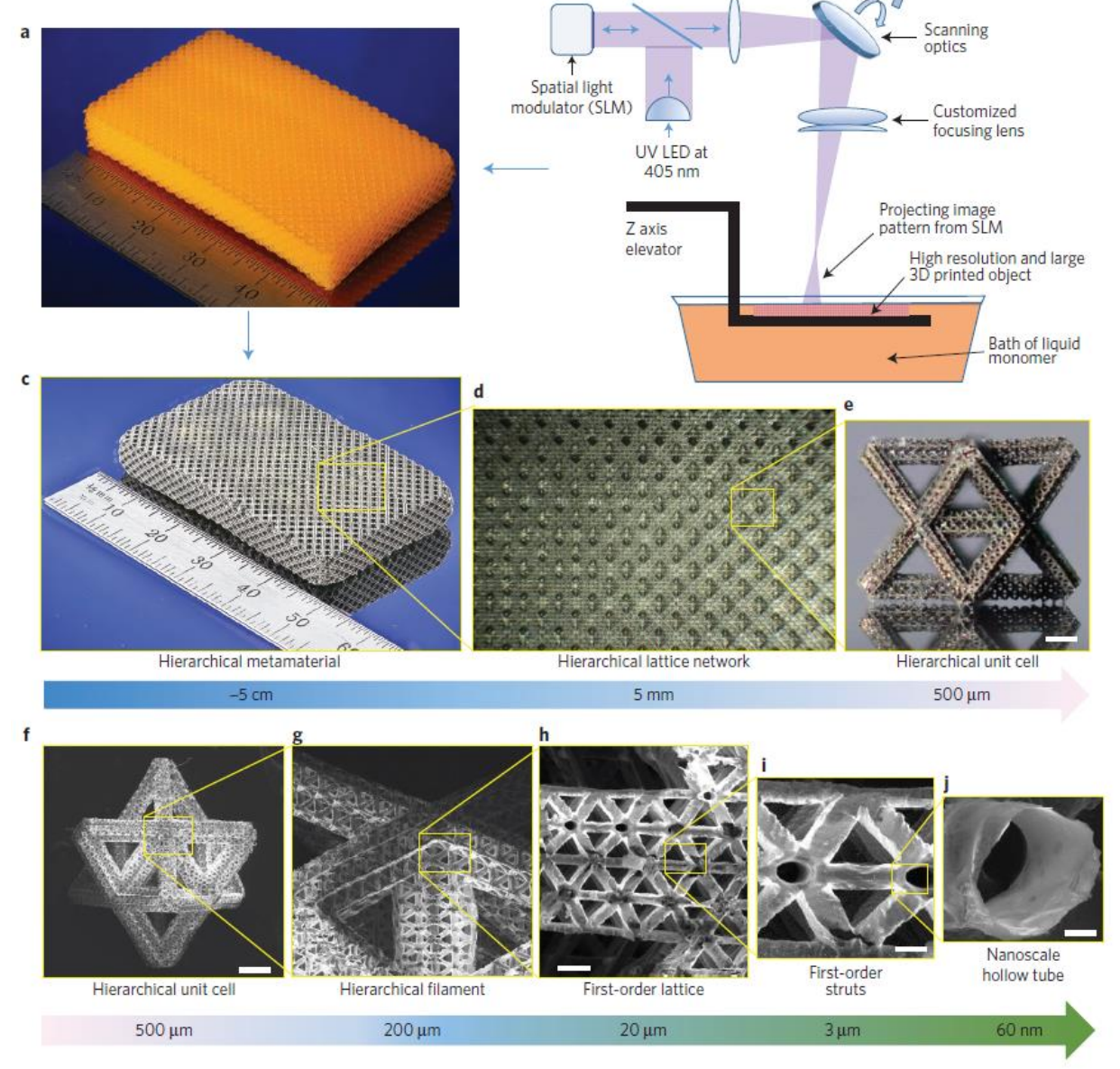

Figure 33 Illustration of fabrication process (b) and breakdown of a structural hierarchy (cj) of the multiscale metallic metamaterial (a)[265].

Jiang and Wang [267] introduced a new class of mechanical metamaterials made from an elastomer with ultra-high reversible stretchability which was four times higher than the existing counterparts (Figure 34). Under large-strain tension, linear scaling of moduli to density was observed while they presented tuneable negative stiffness at large-strain compression enabling ultrahigh-energy absorption. The fabrication process of these highly stretchable metamaterials includes additive manufacturing of a hollow polymer micro-lattice scaffold using projection micro-stereolithography followed by curing elastomers within the hollow tubes and then chemically etching the hollow scaffold leaving the lattice elastomer [267]. 

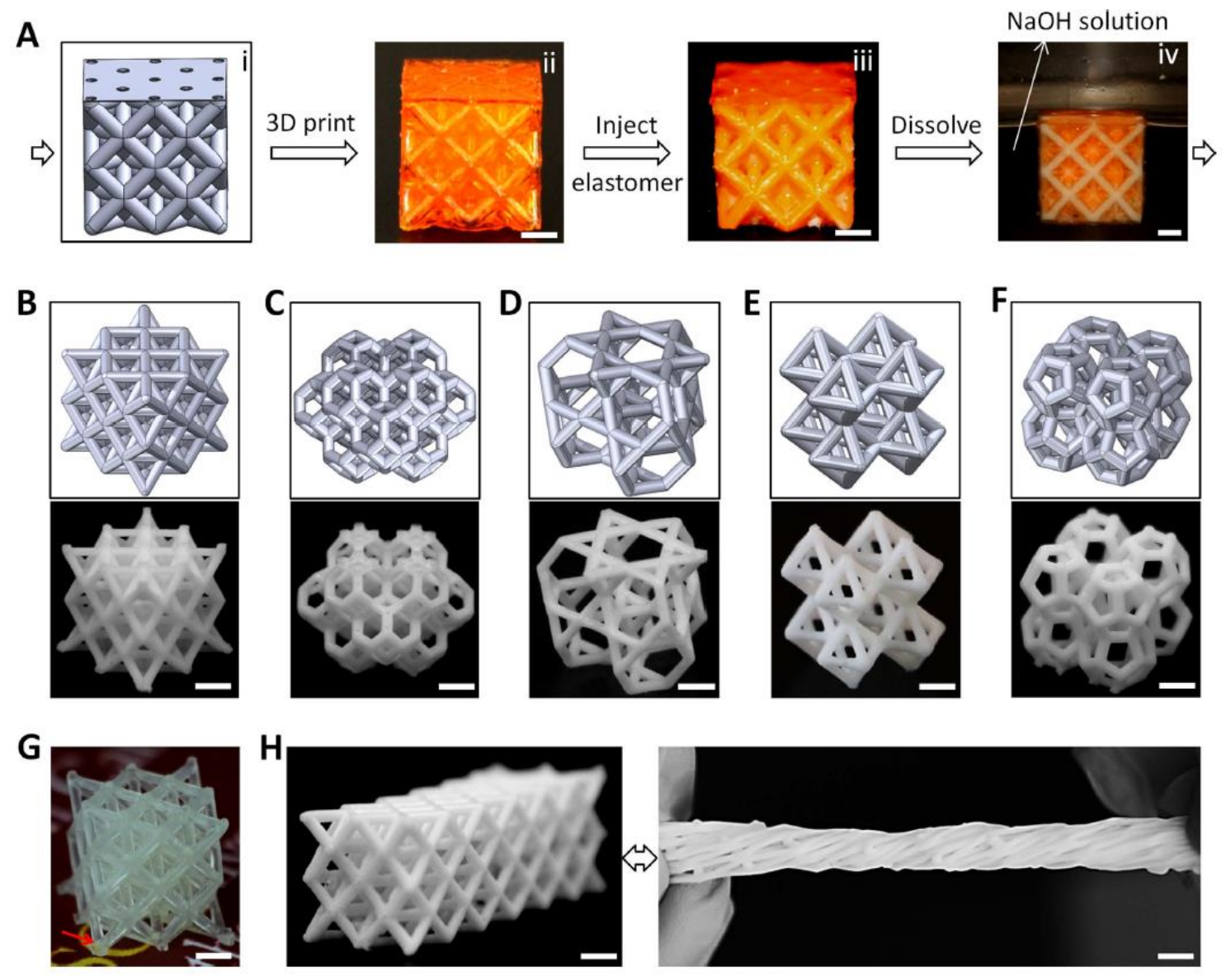

Figure 34 Highly-stretchable mechanical metamaterials made from elastomer [267].

\subsection{SLM}

Selective laser melting (SLM) process has been frequently used for the fabrication of metallic lattice structures for a variety of applications, including automotive [268] and biomedical [242]. Metamaterials used for biomedical applications, so-called meta-biomaterials, were fabricated from biomedical-grade titanium alloy Ti-6Al-4V ELI [242,269]. Mechanical properties of SLM meta-biomaterials were investigated in [269]comparing diamond, truncated cuboctahedron and cubic lattice structures. SLM has also been used for the fabrication of auxetic and pentamode structures [238,242,270]. Hybrid meta-biomaterials were fabricated by a combination of negative and positive Poisson's ratio structures to avoid premature failure caused by tension [242]. The TiNi-based auxetic shape memory alloy structures were fabricated using SLM for potential application as reusable armour [270]. To fabricate metallic pentamode structures, biocompatible and mechanically strong Titanium alloy (Ti-6Al-4V) has been used with SLM technique [238] (Figure 35). The mechanical properties of the fabricated structures were a few orders of magnitude higher than their polymer counterparts. The Young's modulus and strength of the pentamode lattices were decoupled from density, allowing elastic modulus to be independent of permeability which has significant implications in biomedical applications. To produce the pentamode lattice structures with double cone connection 
Hedayati et al. used vector-based energy distribution strategies [238]. Comparison of solid and hollow FCC metamaterials made from stainless steel showed a superior strength and energy absorption capability for hollow lattices when compared against solid lattices with the same density [271].

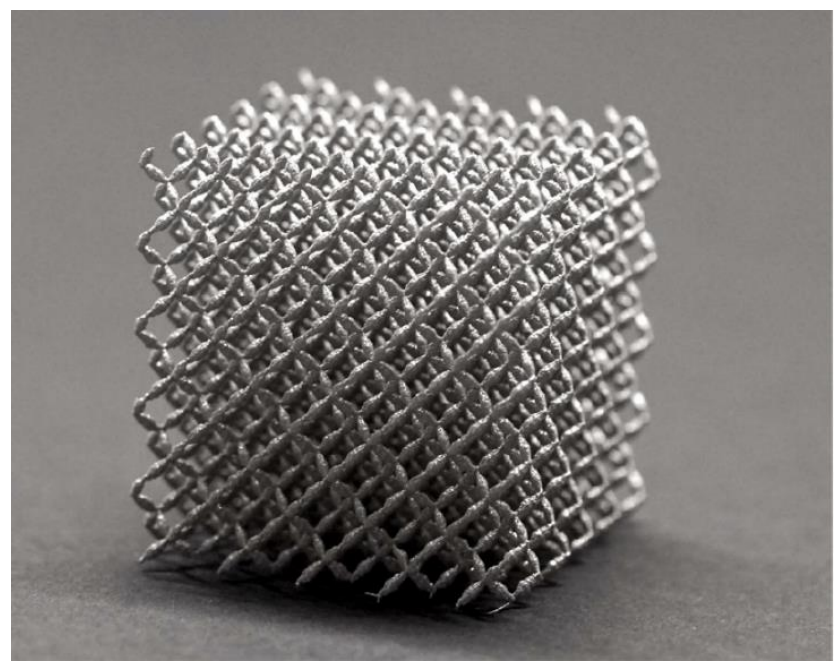

Figure 35 Pentamode Ti-6Al-4V lattice structure made with SLM [238]

\subsection{Selective Electron Beam Melting (SEBM)}

SEBM is a powder bed fusion technique based on the utilization of electron beams to selectively melt the powder bed. This can be used to produce lattice structures with a layer thickness of $50 \mu \mathrm{m}$, and can be used to fabricate a variety of auxetic lattice structures using Titanium alloy (Ti-6Al-4V), for example. Different scan strategies were used in the fabrication of a number of unit cells and the effect of strut size and the filling was tested. By increasing the energy used in melting the powder from 0.4 to $0.8 \mathrm{~J} \mathrm{~mm}^{-1}$ thicker connections were fabricated and the unit-cells were altered. Warmuth et al. [234] reported that the struts dimension has a strong effect on the bulk modulus and Poisson's ratio of the structure. They also reported that the Poisson's ratio is relatively constant as the relative density of structures increases but the elastic modulus increases with the relative density.

Topology optimization technique based on Solid Isotropic Material with Penalization (SIMP) was used to design extremal and auxetic structures with predefined mechanical properties [236] [230]. Traditionally non-manufacturable mathematically optimized structures have been realised using SEBM [236]. To make sure that the optimized structure can be fabricated using SEBM, Schwerdtfeger et al. suggested a hybrid technique based on optimization of structures already fabricated using SEBM and incorporation of special regularization technique in the SIMP. The special regularization technique allows changes that enhance the original design with minimal impact on the ease of fabrication of the part. Using the hybrid optimization technique and an Arcam A2 SEBM system, the Poisson's ratio of $-0.58 \pm 0.04$ was experimentally realized [236]. 
SEBM has also been used in the study of mechanical properties of lattice structures in different directions. Samples with a cell size of $4 \mathrm{~mm}$ were fabricated and mechanically tested. Complete understanding of the behaviour of the auxetic structures helps researchers to be able to tailor the structures to produce the needed mechanical properties [235].

Elastic metallic structures with mesoscopic elements in the range of millimetres have been fabricated using volumetric compression of regular metallic foams which allowed limited control over the resulting structure [236]. Using AM, a significant improvement in the accuracy for metal cellular structures can be achieved [231]. Yang et al., presented an analytical model for the first time to analyse the honeycomb auxetic structures and fabricated these structures using the SEBM technique to compare their numerical and experimental results [231]. To evaluate their formulated numerical simulation, Ti-6Al-4V was used to fabricate the lattice structures and their relative density was measured accurately. Based on the simulation and experiment done by Yang et al. the properties of auxetic honeycomb structures are insensitive to the number of unit cells when this number is greater than or equal to 3 [231].

Using SEBM in the fabrication of structures produces a stepped effect. Therefore, the expected high energy absorption and compressive strength might not be achieved when this phenomenon is present. Fabrication-induced defects are also another source of catastrophic failure in parts [272]. SEBM has been used to manufacture collapsible moulds for fabrication of polyurethane auxetic stents ([232], see Figure 37). Due to the high surface roughness of the SEBM mould, small dents were present on the surface of the auxetic stent sample.
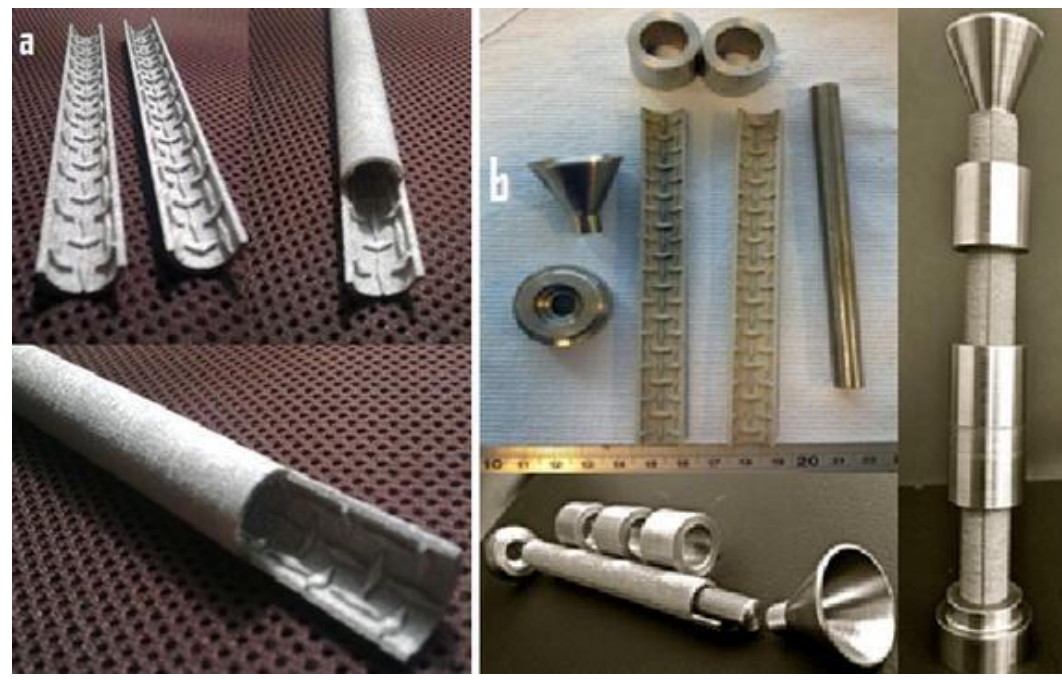

Figure 36 (a) Two halves of the titanium collapsible mould (b) all the components of the mould. The ring, funnel and the internal rod was built of stainless steel. [232]

\subsection{Material Jetting}

3D auxetic metamaterial structures with potential application to energy absorption, vibration control and stretchable electronics have been designed and fabricated using inkjet printing. 
The fabrication of $100 \mathrm{~mm}$ by $100 \mathrm{~mm}$ structures (Figure 37) with beam ligament thickness of $1 \mathrm{~mm}$ [23] has been demonstrated. Auxetic metamaterials with mechanically-tuneable Poisson's ratio were fabricated from a rubber-like material using a multimaterial 3D printer [273]. A macroscopic polymer pentamode metamaterial with bulk to shear modulus ratio as large as 1000 was also printed using "FullCure850 VeroGray" as the polymer [202].

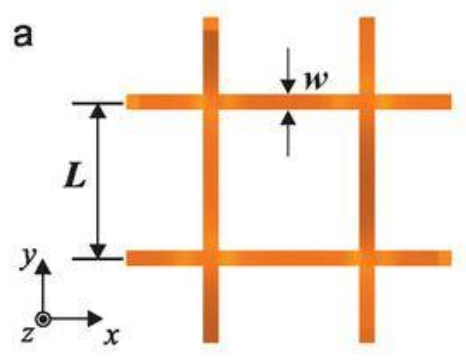

b

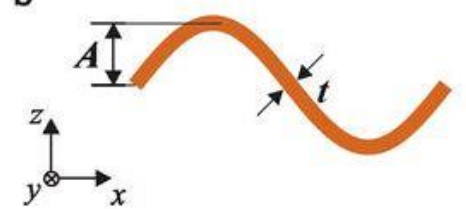

C

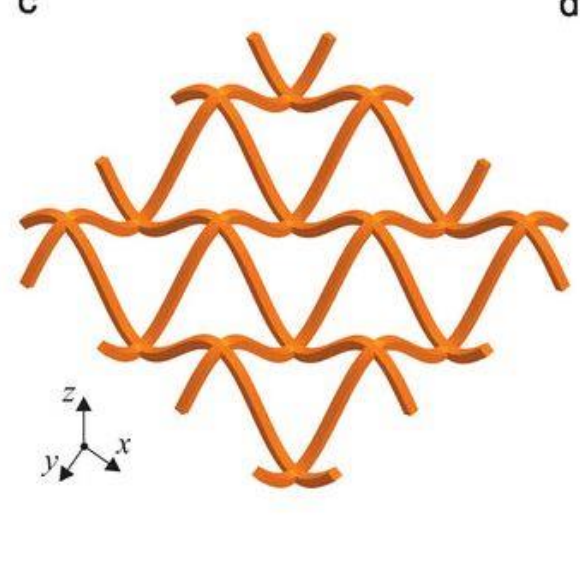

d

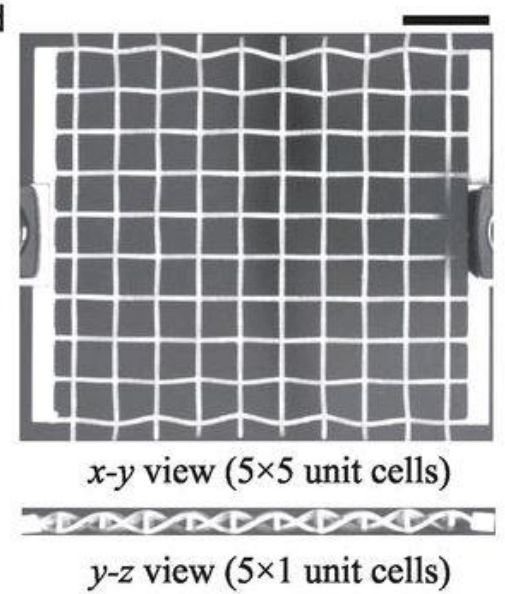

Figure 37: Auxetic metamaterial structure fabricated using material jetting (a) top view (b) side view (c) diagram of proposed construct $(d)$ top view and side view of fabricated structure [23].

Various works have reproduced and tested metallic auxetic structures, but pliable polymers have received little attention. An example is polymeric auxetic re-entrant structures. A Poisson's Ratio of 1.18 has been achieved using commercial Tango Black material to fabricate the lattice structures [237]. The fabricated model was compared to its 3D CAD design and it was concluded that both the damage caused by the potassium hydroxide cleaning solution used, and swelling caused by water penetration into the polymer, leads to high-stress points and fracturing [237].

Mechanical metamaterials have been used in the realization of soft robots that can extend within pipes with a single actuator. The robot is designed based on two soft robotic arms that exert radial pressure to the pipe walls separated by a linear actuator. One of the robotic arms was designed based on auxetic metamaterials and the other printed with positive Poisson's ratio. As the linear actuator press on the arms (see Figure 39), the auxetic structure contracts and it's radial pressure decreases to allow the robot to move. During the contraction phase, this is reversed [22]. 


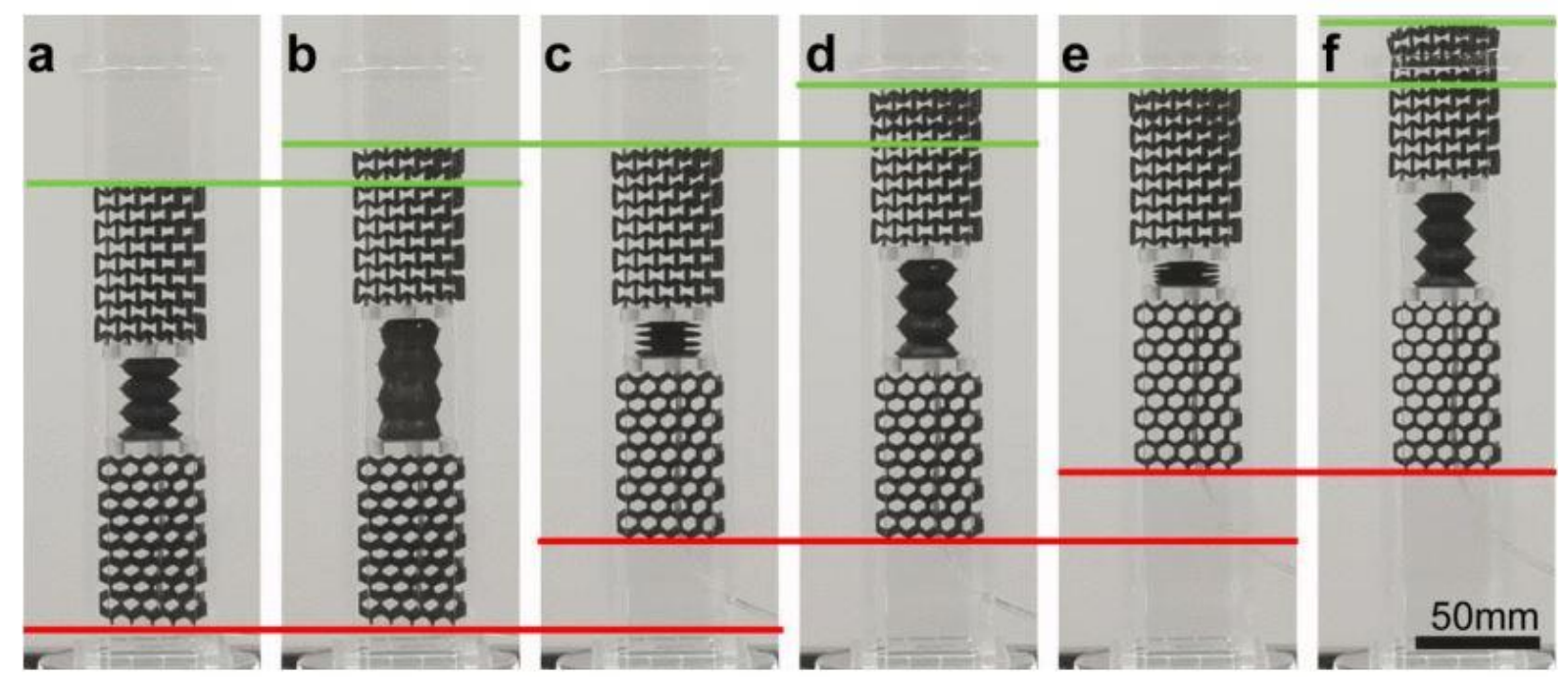

Figure 38: Single actuator soft robot based on one auxetic metamaterial hand [22].

The combination of topology optimization and 3D printing enables researchers to tailor the Poisson's ratio of fabricated structures over large deformation ranges. For example, Clausen et al. [229] used numerical prediction to design 9 different structures, which were printed using silicon-based elastomeric ink (PDMS). Poisson's Ratios of -0.8 to 0.8 were achieved. Although the values of Poisson's ratio were slightly lower than the simulation results, it was thought that this was due to minor deviations in the unit cell structure, which occurs during fabrication.

As mentioned in section 2.2, inkjet printing is a versatile technique and multimaterial structures can be fabricated using this technique. Multimaterial auxetic structures have distinctly different properties comparing to single-material auxetic metamaterials. Wang et al. [274] fabricated dual-material auxetic unit cells using a Polyjet process (Connex Objet350 machine) to demonstrate that dual-material auxetic structures don't have the limitations which are produced by the trade-off between Poisson's ratio and Young's modulus. By using a flexible material for joins and a rigid one for walls/beams, it is possible to produce more stable auxetic structures. The buckling that occurs in the middle of the walls rapidly decreases the auxeticity, but in the dual material auxetic structure, the flexible joints allow stable negative Poisson's ratio for large deformation.

\subsection{Selective Laser Sintering (SLS)}

SLS has been used for the fabrication of macroscopic auxetic materials. Andreassen et al. [230] utilized topology optimization to design 3D materials with periodic structures to achieve Poisson's ratio of -0.5 and bulk modulus of $0.2 \%$ of the solid base materials bulk modulus (Figure 39a). Single material structures presented in this paper were produced in polyamide using an SLS machine [230]. In other work, SLS was used to fabricate 3D soft auxetic lattice structures from dual-segment TPU powders consisting of soft and hard phases [275] (Figure 39b). The auxetic cells included 3D body centre cubic (BCC) and simple cubic (SC) constructed from elastomeric 6-hole or 12-hole spherical shells. Macroscopic polymer auxetic 
structures designed by Bückmann et al. and processed through SLS delivered a Poisson's ratio of -0.8 [233] (Figure 39c).

(a)

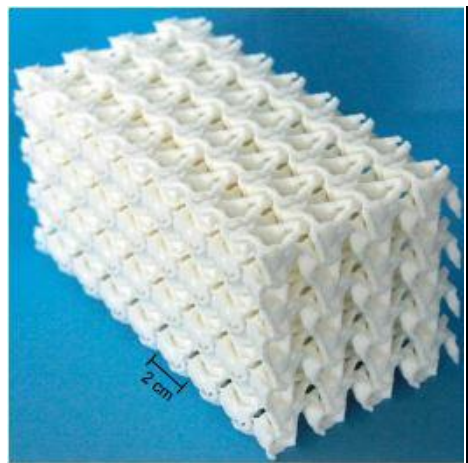

(b)

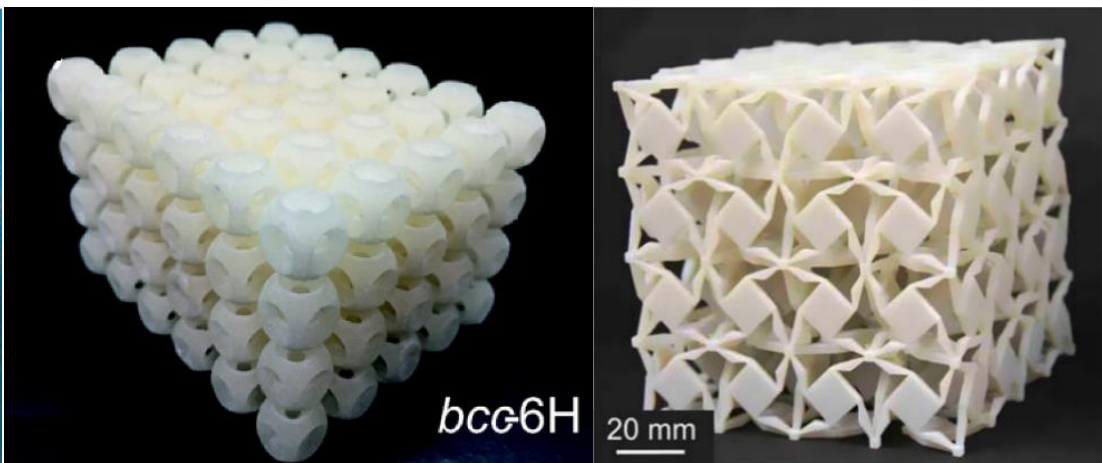

Figure 39 (a) Polyamide structure fabricated using SLS with Poisson's ratio of -0.5 [230] (b) soft auxetic lattice structures made from duel-segment TPU powders [275] (c) Macroscopic polymer auxetic structures with Poisson's ratio of -.08 [233].

\subsection{Fused Deposition Modeling (FDM)}

Rhombohedral and hexagonal unit-cells have been fabricated using soft Polylactide (PLA) material deposited using FDM. A layer thickness of $0.2 \mathrm{~mm}$ and printing speed of $40 \mathrm{~mm} . \mathrm{s}^{-1}$ was used to fabricate the geometries with beam-like members with a length of $16 \mathrm{~mm}$ and a thickness of $1 \mathrm{~mm}$ [276]. The mechanical properties of these structures were studied under both tension and compression in the large deformation regime (Figure 40). They observed that the behaviours of metamaterials are different in axial and transverse direction. The metamaterial response also varies under tension and compression. These observations prove that the metamaterial response is widely dependent on the unit-cell shape, type and size [276]. By introducing different types of cells including shear cells into the design, functional metamaterial mechanisms such as a door latch were fabricated using FDM [277] (Figure 41). Polymer FDM printed meta-sandwich structures made of isomax, octet, cubic and auxetic cells were investigated for failure mechanism, energy absorption and multi-hit capability [278] (Figure 42). 
(a)

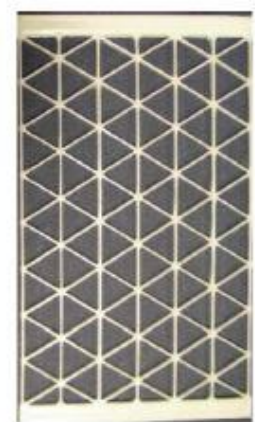

(e)

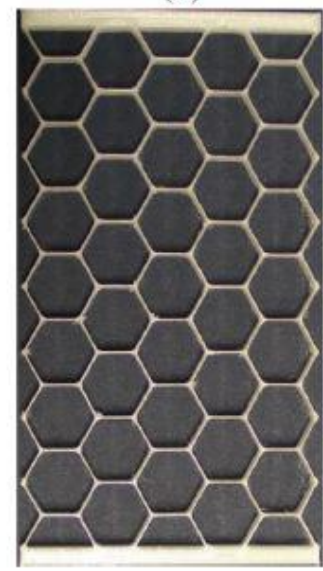

(b)

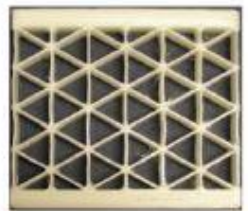

(f) (c)

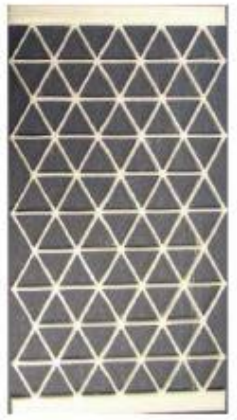

(g)

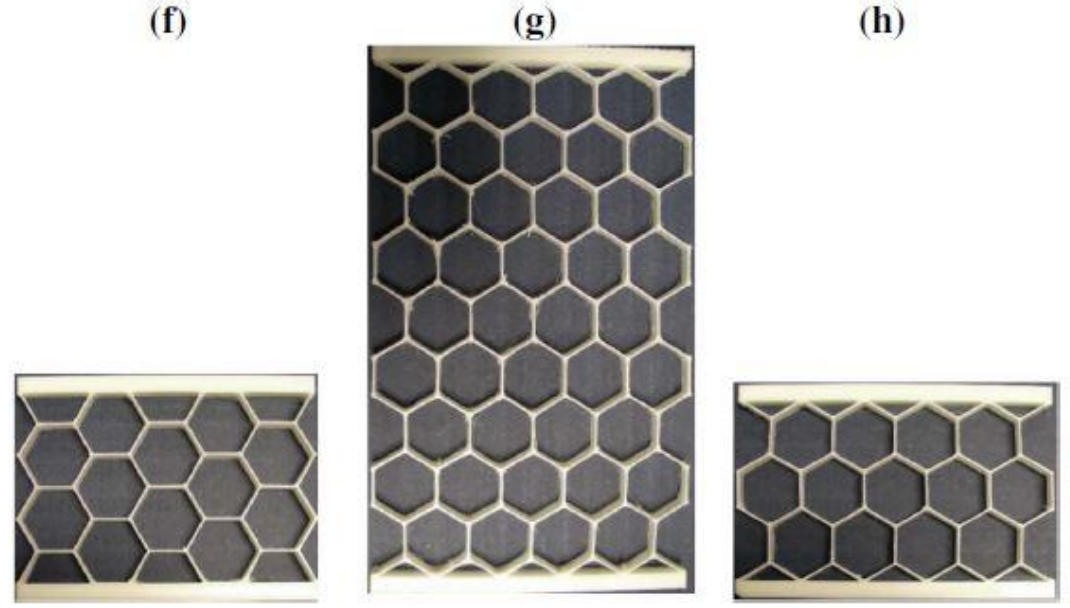

(d)

Figure 40 FDM printed metamaterial structures for mechanical testing. (a,b,e,f) used in axial tests and $(c, d, g, h)$ used for transverse directions. (a,c,e,g) was manufactured for tension test and $(b, d f, h)$ was used in compression testing. Dimensions of samples a- $h$ were $132 \times 86.5 \times 3$, $66 \times 86.5 \times 10,142.2 \times 82.5 \times 3,56.8 \times 82.5 \times 10,228.6 \times 132 \times 3,85.7 \times 132 \times 10,231 \times 143.6 \times 3$ and $82.5 \times 143.6 \times 10 \mathrm{~mm}$, respectively [276].

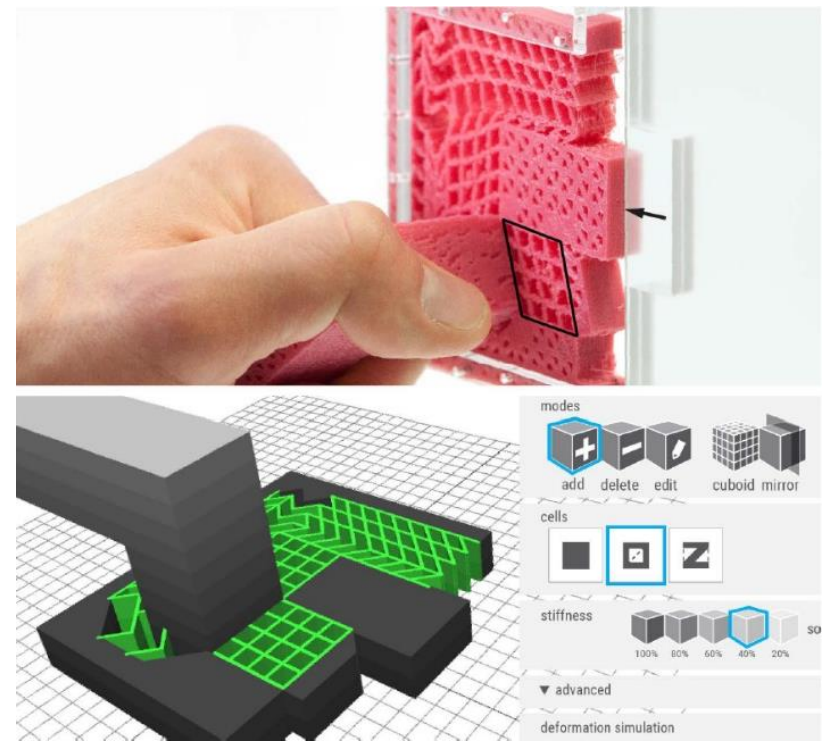

Figure 41 A door latch as a metamaterial mechanism [277]. 


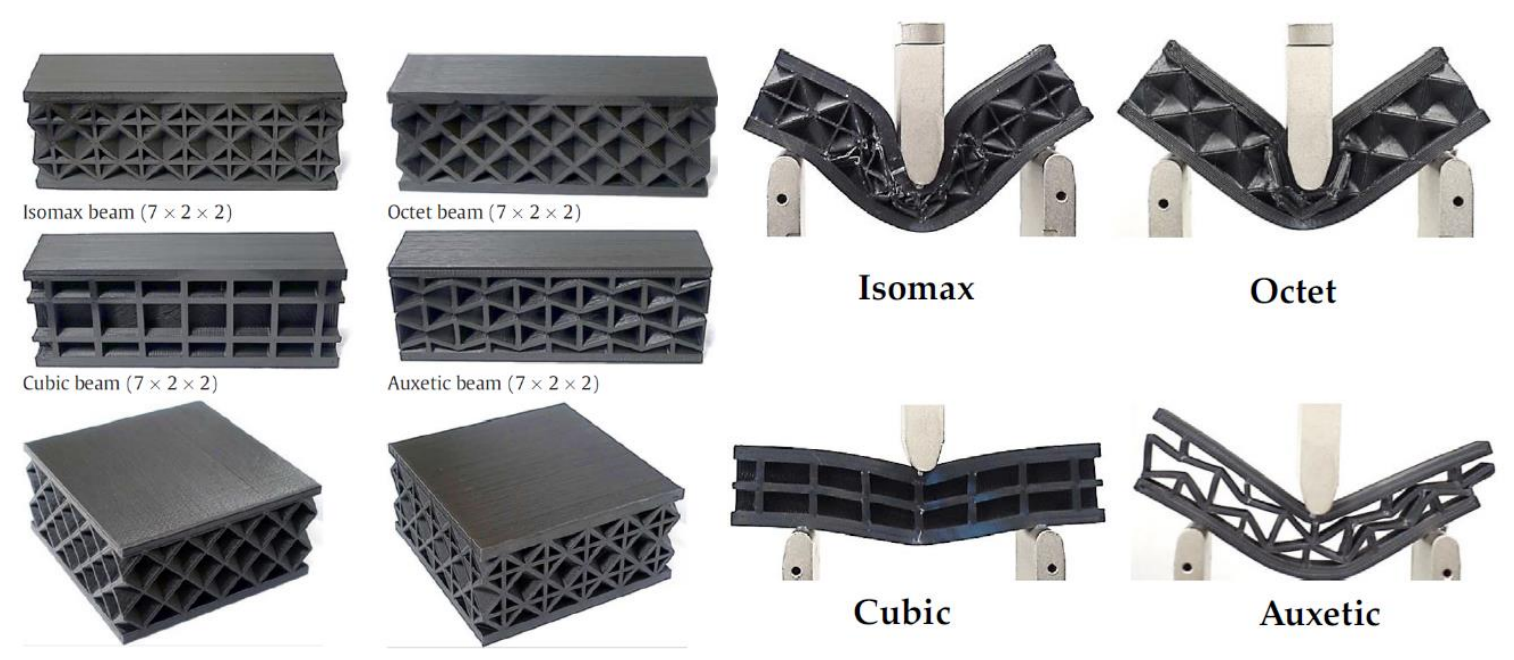

Figure 42 (a) Meta-sandwich structures printed through FDM (b) deformation of metasandwich structures [278].

\subsection{Approaches for bistable metamaterials}

Bistable metamaterials are those where an auxetic material can transform mechanically between two stable states. This has been investigated using structures based on ancient geometric patterns [280] and origami folds [281], where such states arise naturally from their structure. Due to their often complex patterns, such metamaterials lend themselves to additive manufacturing as opposed to conventional manufacturing methods. FDM has been the most commonly-used method for such fabrications. This has been demonstrated by Rafsanjani et al. [282], where a nylon-based material under tension exhibited a snapping behaviour between two states. This is an example of non-linear behaviour, in contrast to other mechanical materials where the behaviour is mostly assumed to be linear. There are many potential applications for such materials, including vibration isolation and damping. A material with multiple bistable cells has also been investigated by Che et al. [283], with a design that allows for adjustable mechanical properties by using mixed multiple resins to produce the final structure with the correct mechanical properties.

Recent work has demonstrated that the bistability can be extended to multiple states [284]. Such materials have also been fabricated via FDM. Here two structures were built which were then combined so that the composite structure exhibited the behaviour of interest. The authors suggest that applications such as biomechanics could benefit from the resultant properties, as interesting mechanical and shape changes are possible as a function of applied stress. Structures based on traditional origami paper-folding techniques have also been explored, where the stiffness of a unit cell can be changed by bistable switching; however, if a stacked sequence of multiple cell designs are used, the overall properties can be tailored to a specific response. This was demonstrated by Sengupta and Li [285] and fabricated in a thermoplastic elastomer using 
SLS. Such structures have many potential uses in morphing structures and soft robotics by switching between stable states, each of which having a predictable elastic modulus [286].

Smaller unit cells which also exhibit 'snapping' between states have been fabricated using multi-photon lithography (MPL) [287]. Here, the aim was to demonstrate the possibility of structures exhibiting tensegrity (tensional integrity), which use bars or struts under compression within a network of other struts in continuous tension, and where the compressed members are not in contact with each other. The resulting structure had interesting dynamic mechanical operation properties in terms of softening and stiffening deformation mechanisms.

Finally, twisting and rotational bistable matamaterials were recently fabricated via inkjet in such way that no post-assembly was needed [288]. To this aim, special joints were placed to construct those devices in a single AM printing step without affecting the intended properties.

\section{Multiple Material Approaches}

Sequential material deposition processing or hybridised multi-material processing allows for new approaches in design this is enabled by recent advances to both process and feedstock. Indeed, strategies making use of multiple materials are being increasingly considered for the fabrication of metamaterials. Multi-material metamaterials create a wider range of material design freedoms over and above those obtained from geometry alone. Multi-material additive manufacturing is a highly researched topic with innovations across the seven broad categories of AM and is key to providing both geometrical complexity and material flexibility over one structure. By way of example, multi-material AM can be realised by adding multiple print heads in inkjet or material extrusion processes, by introducing secondary material vats in vat photopolymerisation, or by adding multiple powder streams for DED. These constitute the primary methods for multiple material approaches. However, here, the three primary routes to multi-material deposition in AM used to fabricate metamaterial structures: inkjet, material extrusion, and vat photopolymerisation are considered. Readers are referred to [289] for further information on multi-material AM in general, and to work in the metals and ceramics space in directed energy deposition [290] and powder bed fabrication [291].

Inkjet printing is used in multi-material metamaterial studies as commercial multi-material inkjet printers are now readily available. These processes are complimented by material availability such as UV curable photopolymer materials with varying properties such as high tensile strength, flexibility, biocompatibility, and materials with different colours and opacities. Wang et al. [292] used a Polyjet to create an auxetic metamaterial made from Vero White Plus ${ }^{\circledR}$ (a stiff material) with Tango Black Plus ${ }^{\circledR}$ (a flexible material) at the joints of a re-entrant structure. The parts tended to fail before the theoretical strain limits attributed to defects during printing due to inclined beams. Build orientation is known to affect the quality of parts due to the layer-by-layer fabrication process, investigating different orientations for this part may help alleviate any defects on the inclines.

Wang et al. also [291] and [292] used a Polyjet ${ }^{\circledR}$ system to embed stiff structures into a soft polymeric matrix in a similar manner. They concluded that stiff double helix structures could be used to mimic elastin-rich biological tissues however this is based on assumptions of the stress strain curve past the $8 \%$ strain mark where measurements were capped due to the material interfaces breaking down at $9 \%$ strain [293]. The addition of an adhesive material at the interface may help with this, however adding a third material will increase fabrication complexity, and may adversely affect the resulting properties. Features created here are also limited to $0.1 \mathrm{~mm}$ due to the printer resolution, however this limitation could be overcome by 
using printing technology with a higher resolution which is available. Sitthi-Amorn et al. [293] created a home-built inkjet system capable of reaching feature sizes of $40 \mu \mathrm{m}$ or less. Although this figure is dependent on the aspect ratio of structures and the materials used. Moreover, the printer has sacrificed fabrication speed for this increase in resolution.

Mirzaali et al. [294] created multi-material metamaterials by fabricating each unit cell as one of two materials; VeroCyan ${ }^{\circledR}$ as the hard material and Agilus30 Black ${ }^{\circledR}$ for the soft material. Finite element models were created where soft elements were randomly replaced by VeroCyan ${ }^{\circledR}$ up to specific fractions and modelled computationally. Randomly assigning $50 \%$ of the unit cells with VeroCyan showed approximately 2 orders of magnitude increase in the elastic modulus. The high mismatch, $E_{\text {hard }} / E_{\text {soft }} \approx 1000$, between the hard and soft materials created local stresses which can result in bucking or cracking over repetitive loading leading to pre-mature failure. However, authors suggest using intermediate phases with carefully selected mechanical properties to decrease the severity of the stress concentrations. Indeed, Saxena et al. [297] computationally studied gradients of four materials from hardest $(\mathrm{E}=$ $2300 \mathrm{MPa})$ in the middle $40 \%$ of the beams to softest $(\mathrm{E}=0.5 \mathrm{MPa})$ at the joints (the intermediaries were $\mathrm{E}=1.4 \mathrm{MPa}$ and $0.8 \mathrm{MPa}$ ); the three softer materials made up the remaining $60 \%$ of each beam. They found the distribution pattern in the amounts of each intermediary material to not have a significant effect on the Poisson's ratio. Stresses were shared across the interfaces between each material addressing the issues presented in [295].

Hybrid chiral and re-entrant structures to be used as opening mechanisms were developed in [296]. Using Polyjet ${ }^{\circledR}$ technology, the bulk of the structures were fabricated from DM9760 ${ }^{\circledR}$ and a stiffer material (VeroWhite ${ }^{\circledR}$ ) is used for the connecting ribs in the re-entrant core cells to reduce the bending of the connections increasing auxetic performance. An alternative single material design showing similar mechanical properties was presented in the supplementary material where the connecting rods were thicker than the rest of the structure. This was included as an alternative for where multi-material AM is not available, as while not specifically mentioned, the cost of commercial multi-material machines and their materials can be prohibitive.

In [297], composite structures made from various fill fractions of glassy and elastic polymers were shown to create different failure mechanisms to enhance energy dissipation. Experimental yield stresses were slightly lower than simulations attributed to the fidelity of the printing process, a common theme across $3 \mathrm{D}$ printing. The printer resolution was quoted to be $\sim 30 \mu \mathrm{m}$ but inaccuracies can affect the volume fraction and distribution of each material leading to discrepancies.

Saleh et al. [298] used a duel head inkjet printer to print both passive (EMD6415 ${ }^{\circledR}$ from SunChemical) and conductive (EMD6145 ${ }^{\circledR}$ with iron oxide nanoparticles) inks in the form of "waffle" structures for RF applications. Printable inks should have a $\mathrm{Z}$ number between $1<\mathrm{Z}<10$ where $\mathrm{Z}$ is the inverse Ohnesorge number $(\mathrm{Oh})$, and $\mathrm{Oh}$ relates the internal viscosity dissipation to the surface tension energy. Adding the iron oxide to the EMD6415 ${ }^{\circledR}$ increased the ink viscosity. To address this, a binder solution was formulated from $95 \mathrm{wt} \%$ EMD6415, $2 \mathrm{wt} . \%$ ethylene glycol dimethacrylate to decrease viscosity, and 3 wt.\% of BYK-UK-3570 ${ }^{\circledR}$ surfactant $\left(\right.$ ALTANA $\left.^{\circledR}\right)$ to sustain nanoparticle dispersion. From this, two conductive inks were developed having $5 \mathrm{wt} . \%$ and $10 \mathrm{wt} . \%$ copper oxide nanoparticles suspended in $95 \mathrm{wt} . \%$ and $90 \mathrm{wt} . \%$ of the binder which resulted in $\mathrm{Z}$ numbers of 1.72 and 1.57 respectively. A third $20 \mathrm{wt} . \%$ copper oxide ink was investigated but had viscosity that was too high to print. In addition, UV penetration is reduced with high nanoparticle loadings reducing the curing of the polymer, placing an upper limit on the nanoparticle percentage. This limitation could be resolved through varying chemical formulation.

While multi-material printing technologies exist, making the most of the extended range of functionalities can be difficult if relying on intuitive design. In this way, topology optimisation 
is a useful tool in designing structures with specific desired properties. Vogiatzis et al. [299] used a reconciled level set method to design multi-material auxetic structures that were printed via inkjet. By using level set representation, this least square minimisation method finds the steady state solution of the Hamilton-Jacobi equation for the required Poisson's ratio, thus finding the optimal material layout of the hard and soft phases. The work found good agreement between simulations and experiments. However, authors noted the difficulties in removing supporting material from the manufactured structures and said future works will take AM constraints into account for the topology optimisation design process. Again this is a practical consideration the AM practitioner must address.

Material extrusion can also be used to create multi-material parts and there are commercial machines available for this. Two categories of materials can be extruded which are either melted thermoplastic polymers or paste-like inks that can be metal, ceramic or polymer. Thermoplastic filaments can be altered to change their properties like in Isakov et al.'s work [300]. Resonant structures which had alternating layers of polypropylene (PP), and a $\mathrm{CaTiO}_{3} / \mathrm{PP}$ composite were created using duel filament FDM. Creating new filaments that print without blocking, filament breakage, and layer merging requires a balance between stiffness, surface finish, small diameter variations, and adhesion behaviour. Due to this they found polymer printability was limited to less than $30 \mathrm{vol} \%$ of ceramic [301]. Additionally, results showed experimental resonances were less pronounced than the simulation due to imperfections in the printing process creating trapped air/voids in the structure. This can be addressed by looking into optimum processing parameters or creating a hybrid system that can add an adhesive between different materials.

The accuracy of FDM can be considered a limiting factor for metamaterial development as geometrical fidelity is important to the resulting properties. Momeni et al. [302] investigated sensitivities to printing deviations in single and multi-material high strength octet-truss structures using PLA and tough-PLA. Results found that the bi-material unit cells had less variation in their elastic moduli than the single material unit cells showing less sensitivity to deviations during printing. For the bi-material structures, the stored elastic energy is independent of the number of materials used but is increasingly sensitive to variations in the diameter of the exterior elements as the displacement increases. Therefore, it is important to be mindful of printing parameters when creating multi-material structures where a balance between accuracy and printing speed should be found.

Gheisari et al.'s work [303] used a slurry-based extrusion method coupled with selective laser burnout (SLB) to create a ceramic/metal mutli-material RF metamaterial. In this work a $\mathrm{Bi}_{2} \mathrm{Mo}_{2} \mathrm{O}_{9} 81$ wt.\% ceramic slurry was prepared as a substrate for a 90 wt.\% Ag paste transmission line. Each layer was extruded before being scanned with a low energy density laser, burning out the organic components leaving only the ceramic / metal. The SLB process reduces shrinkage lessening cracking allowing for more complex designs, it also increases dimensional accuracy during fabrication. However, the laser energy density must be carefully selected so that it is high enough for the solvent and binder to be fully removed while not being too high so as to cause not to cause local melting which destroys the geometry. This required looking into combinations of laser power and scan speed that produce good parts within a reasonable time. The SLB process also reduces undesirable reactions at the interface between the metal and the ceramic which can negatively affect RF properties. They suggest using an alternative slurry that does not react with Ag as a solution to this [304].

Vat photopolymerization presents problems in creating multi-material parts, usually due to cross-contamination of different materials. Nonetheless, purpose built machines have been used to create multi-material metamaterials. Wang et al. 2016 [305] used multi-material projection-MSL to create PEGDA/PEGDA-Copper structures exhibiting a negative thermal expansion (NTE). The NTE was varied by changing the copper concentration from 2 vol. $\%$ to 
$10 \mathrm{vol} . \%$. In their work $10 \mathrm{vol} . \%$ was deemed the upper concentration limit for reasonable print times of 6 hours for each structure. Printing time increases with higher concentrations of copper nanoparticles setting a lower limit on the thermal expansion coefficient, higher concentrations also decrease the bonding force between the printed layers. Upper limits on particle inclusions have been seen throughout this section and may be resolved in part through different chemical formulations, however at present is a fundamental material limitation.

Chen and Zheng [306] also used projection-MSLA to manufacture multi-material auxetic metamaterials. This system aimed to address the issue of cross contamination by integrating a cleaning step when switching materials. While the robotic cleaning system can switch materials in 10s including cleaning and drying time, if multiple materials are used in one layer this can add a significate time to the resulting print depending on the size and number of layers required. This may be considered a necessary trade-off for creating multi-material metamaterials with resolution down to $5 \mu \mathrm{m}$ with the current state-of-the-art.

In [307] optical trapping was combined with multiphoton polymerisation to create polymer fishnet structures with particles arranged inside the gaps. The set up can be used to position a wide number of particles provided their refractive index is higher than that of the photoresist. Fabrication speed is limited by the trapping force generated by the OT, so to increase fabrication speeds a diffractive optical element (DOE) was used to produce 7 traps in a hexagon pattern allowing an increase in fabrication speeds. Accuracy of the particle deposition is controlled by the focal spot of the polymerisation laser; by reducing the spot size less polymer is used to fix the transported particle in place reducing any movement of the particle during polymer solidification.

A hybrid MMP and LIT system by Kuznetov et al. [308] allows spherical nanoparticles to be deposited onto specific areas of a design. The particles change their shape according to the receiver structure allowing different designs to be realised. One limitation is that a femtosecond laser is used for both LIT and photopolymerization so the LIT process cannot be done in the presence of photoresist. Therefore, this is a sequential process with increased fabrication times, but may be an acceptable compromise to be able to realise metal/polymer structures at the nanoscale.

In summary multi-material AM is a fast-growing area of research. As this section has demonstrated there are limitations that should be considered when designing multi-material metamaterials using AM techniques. There are stark process and material limitations which represent even greater challenges in some cases as compared to

\section{Conclusions and Directions for Future Research}

The literature presented here provides evidence of tremendously active interaction between the metamaterials and additive manufacturing research communities. In recent years, these areas have interacted most productively to exploit successive innovations. Through fundamental understanding of material behaviour, enriched by advanced modelling and design tools, alongside an ability to realise physical approximations of mathematically ideal geometries our ability to exploit metamaterials is accelerating. This review describes the use of AM methods for the creation of new and exciting metamaterial structures which otherwise could not have been built. It is to be expected that research in this area will continue to create new structures with exotic properties and imposing limitation from this vantage point would be foolhardy.

In all examples presented here, where computational design and modelling have been used in the exemplar literature, the manufacturing methods used fall short of maintaining $100 \%$ fidelity 
to the theoretical design intent. Loss of fidelity to design intent can be attributed to inconsistencies in 'bulk' materials properties, or geometrical artefacts introduced during the manufacturing process. On several occasions within the literature, superior material/structural properties have been predicted but performance has been limited due to the lack of precision achievable by layer-based techniques. Furthermore, the materials properties associated with AM processes are often inconsistent with properties resulting from traditional manufacturing processes. As such there remains significant opportunity for fundamental research in enhancing process precision and modulating bulk properties. Advances here will allow the engineer to deliver structures which better represent the physicist's specification.

Much of the literature presented relates function of metamaterials to the resolution of the features which constitute them. This in turn limits the domains over which useful phenomena can be observed. Hence there is a clear and present need to not only enhance process technologies for enhanced resolution but also enhance the scalability of these. This is perhaps best exemplified by two photon polymerisation. While near optically relevant $(<1 \mathrm{um})$ features can be created, the rate of production and therefore the scale over which these features can be formed is limited. As a result there is tremendous opportunity to further enhance resolution and scale up process technologies.

The pallet of materials for additive manufacturing (in both in/organic) has expanded rapidly in recent years [48]. However, there remains significant opportunity for new materials innovation which meets requirements for both i) suitability to process and ii) function. Indeed material transformations which take place during process (e.g. recrystallization in SLM) are still underexplored and the utility of these to exotic applications such as metamaterials is yet to be understood.

Opportunities presented by utilization of AM in the fabrication of metamaterials can be classified in two main categories. The first category is the chance to mainstream the use of metamaterial by decreasing the production cost and enabling the incorporation of metamaterials in everyday structures, hence increasing the applications of metamaterials. AM can greatly increase the production capability and decrease the manufacturing cost for complex geometries needed for metamaterials. Techniques such as material jetting can be used in the fabrication of complex structures on flexible substrates, and this can satisfy the durability requirements for everyday use. Inkjet printing is also scalable and using a high number of ink cartridges with hundreds of nozzles the system throughput can greatly increase. Combining AM techniques with well-established roll to roll processing technology can potentially move the metamaterial fabrication from laboratories to the factory floor.

The second avenue that metamaterials can greatly benefit from the development of AM techniques is in high complexity $3 \mathrm{D}$ structures. The true $3 \mathrm{D}$ fabrication capabilities of $\mathrm{AM}$ relax some of the design and fabrication limitations imposed by subtractive manufacturing and 2D patterning techniques. Consequently, AM enables the researchers to fabricate and study structures which were not possible to build using conventional manufacturing. The resolution and repeatability of AM techniques are increasing and techniques such as MPP can fabricate 
sub-micron structures with resolution comparable to lithographic techniques. Micro-SLA and SIJ techniques can fabricate structures in multiple length scales and their relatively large fabrication area give them an added advantage in metamaterial fabrication. In contrast to lithographic techniques, AM techniques can function in a normal room environment and can be used in the fabrication of nested 3D structures with high resolution and repeatability.

Fabricating metamaterials by the means of AM is still in its early stages and a number of challenges limit the application of these techniques. Material availability is one of the main issues. Each AM technique has a unique material requirement which complicates the material development process. Researchers are continuously trying to develop new materials. For metamaterial applications, newly developed materials should also have beneficial properties for their respective applications. A similar requirement is present in the acoustic metamaterials and the fabricated structures should have higher acoustic impedance compared to the environment that they are operating in. Therefore, there is a need for further research on the production of new or modification of existing AM materials that meet the requirements for different metamaterial application.

It is apparent that multi-material AM techniques are essential for widespread application of AM techniques in the fabrication of different types of metamaterials. The majority of AM techniques are single material technique and to fabricate hybrid structures the AM techniques should be combined with another procedure. In order to increase the applications of AM in the fabrication of metamaterials, new multi-material AM techniques should be developed to be able to fabricate materials with different mechanical, acoustic and optical properties.

Post-processing of AM parts, such as support removal and development, often affect the fabricated structure. Shrinkage, fractures and rough surface finish are some of the issues that occur during the post-processing stage of 3D printed parts. Due to the high sensitivity of metamaterials to structural modification, a small change can greatly reduce the effectiveness of the metamaterial structure. To tackle this issue, the deformations produced during each process should be compensated for in the design stage. Therefore, the design process is likely to become more complex and dependent on the specific manufacturing techniques chosen. The challenges facing fabrication of metamaterials using AM techniques call for the development of innovative AM techniques capable of fabricating structures with multiple length scales.

In summary the authors have attempted to provide a comprehensive overview of the state-ofthe-art in a hugely important research area. At the time of writing the research community is producing approximately 10 papers per day relating to metamaterials (see Figure 1). As a result, this modest review will become dated rapidly. Yet we would be so bold as to highlight the principle research challenges and the domains which will address these below.

Table 1 The primary research challenges for advancing AM technology for the realisation of metamaterials and the domain from which solutions may be drawn. 


\begin{tabular}{l|c|c|c|}
\multicolumn{1}{c|}{ Research Challenge } & \multicolumn{2}{c|}{ Solution Domain } \\
\hline To enhance fidelity to design intent & Metamaterial Design/Modelling & AM Process Development & Materials Science \\
\hline To increase build rate & $\mathrm{X}$ & $\mathrm{X}$ & $\mathrm{X}$ \\
\hline To enhance resolution & & $\mathrm{X}$ & $\mathrm{X}$ \\
\hline To improve part integrity & $\mathrm{X}$ & $\mathrm{X}$ \\
\hline To increase complexity of multi-material systems & $\mathrm{X}$ & $\mathrm{X}$ \\
\hline To liberate more design freedom & $\mathrm{X}$ & $\mathrm{X}$ \\
\hline
\end{tabular}

It is evident that the advent of new metamaterials facilitated by AM technology will provide opportunities across research areas and impact greatly upon products of all kinds. However, our mastery of these technologies is limited. Advances made thus far have largely been achieved through cooperation across disciplines. In this field of metamaterials creation the physicist, the chemist, the mathematician, the engineer, the materials science and the biologist may find plentiful opportunity for discovery.

\section{Acknowledgements}

Funding for this work was provided through the UK Engineering and Physical Sciences Research Council (EPSRC), Grant numbers EP/N034163/1, EP/N034201/1 and EP/N034813/1.

\section{References}

[1] W. Cai, V. Shalaev, Optical metamaterials: Fundamentals and applications, Springer New York, 2010. https://doi.org/10.1007/978-1-4419-1151-3.

[2] J. Valentine, S. Zhang, T. Zentgraf, E. Ulin-Avila, D.A. Genov, G. Bartal, X. Zhang, Three-dimensional optical metamaterial with a negative refractive index, Nature. 455 (2008) 376-379. https://doi.org/10.1038/nature07247.

[3] N. Engheta, R.W. Ziolkowski, Metamaterials: Physics and Engineering Explorations, John Wiley \& Sons, Inc., Hoboken, NJ, USA, 2006. https://doi.org/10.1002/0471784192.

[4] V.M. Shalaev, W. Cai, U.K. Chettiar, H.-K. Yuan, A.K. Sarychev, V.P. Drachev, A. V. Kildishev, Negative index of refraction in optical metamaterials, Opt. Lett. 30 (2005) 3356. https://doi.org/10.1364/OL.30.003356.

[5] Y. Xie, S. Ye, C. Reyes, P. Sithikong, B.I. Popa, B.J. Wiley, S.A. Cummer, Microwave metamaterials made by fused deposition 3D printing of a highly conductive copper-based filament, Appl. Phys. Lett. 110 (2017) 181903. https://doi.org/10.1063/1.4982718.

[6] M. Zedler, C. Caloz, P. Russer, A 3-D isotropic left-handed metamaterial based on the rotated transmission-line matrix (TLM) scheme, IEEE Trans. Microw. Theory Tech. 55 (2007) 2930-2941. https://doi.org/10.1109/TMTT.2007.909608.

[7] J. Zhu, J. Christensen, J. Jung, L. Martin-Moreno, X. Yin, L. Fok, X. Zhang, F.J. Garcia-Vidal, A holey-structured metamaterial for acoustic deep-subwavelength imaging, Nat. Phys. 7 (2011) 52-55. https://doi.org/10.1038/nphys1804.

[8] T. Skulavik, M. Kopcek, A. Kopcekova, Fuzzy control of robotic arm implemented in 
PLC, ICCC 2013 - IEEE 9th Int. Conf. Comput. Cybern. Proc. 93 (2013) 45-49. https://doi.org/10.1109/ICCCyb.2013.6617628.

[9] E. Yablonovitch, T.J. Gmitter, K.M. Leung, Photonic band structure: The facecentered-cubic case employing nonspherical atoms, Phys. Rev. Lett. 67 (1991) 22952298. https://doi.org/10.1103/PhysRevLett.67.2295.

[10] M.K. Hedayati, M. Javaherirahim, B. Mozooni, R. Abdelaziz, A. Tavassolizadeh, V.S.K. Chakravadhanula, V. Zaporojtchenko, T. Strunkus, F. Faupel, M. Elbahri, Design of a perfect black absorber at visible frequencies using plasmonic metamaterials, Adv. Mater. 23 (2011) 5410-5414. https://doi.org/10.1002/adma.201102646.

[11] M. Hermatschweiler, A. Ledermann, G.A. Ozin, M. Wegener, G. Von Freymann, Fabrication of silicon inverse woodpile photonic crystals, Adv. Funct. Mater. 17 (2007) 2273-2277. https://doi.org/10.1002/adfm.200601074.

[12] Fabrication and characterization of silicon woodpile photonic crystals with a complete bandgap at telecom wavelengths, Opt. Lett. 35 (2010) 1094-1096. https://doi.org/10.1364/OL.35.001094.

[13] J.D. Joannopoulos, P.R. Villeneuve, S. Fan, Photonic crystals: Putting a new twist on light, Nature. 386 (1997) 143-149. https://doi.org/10.1038/386143a0.

[14] R.W. Ziolkowski, E. Heyman, Wave propagation in media having negative permittivity and permeability, Phys. Rev. E - Stat. Physics, Plasmas, Fluids, Relat. Interdiscip. Top. 64 (2001) 15. https://doi.org/10.1103/PhysRevE.64.056625.

[15] C. Caloz, Metamaterial Antennas and Radiative Systems, in: Y.M.. A. Debatosh Guha (Ed.), Microstrip Print. Antennas New Trends, Tech. Appl., 1st ed., Wiley \& Sons Ltd, 2010: pp. 345-386. https://doi.org/10.1002/9780470973370.ch11.

[16] A. Di Falco, M. Ploschner, T.F. Krauss, Flexible metamaterials at visible wavelengths, New J. Phys. 12 (2010) 113006. https://doi.org/10.1088/1367-2630/12/11/113006.

[17] OLD Broken, G. Ma, P. Sheng, OLDBROKENAcoustic metamaterials: From local resonances to broad horizons, 2016. https://doi.org/10.1126/sciadv.1501595.

[18] A. Khelif, A. Adibi, Phononic crystals: Fundamentals and applications, Phononic Cryst. Fundam. Appl. (2015) 1-245. https://doi.org/10.1007/978-1-4614-9393-8.

[19] A. Kruisová, M. Ševčík, H. Seiner, P. Sedlák, B. Román-Manso, P. Miranzo, M. Belmonte, M. Landa, Ultrasonic bandgaps in 3D-printed periodic ceramic microlattices, Ultrasonics. 82 (2018) 91-100. https://doi.org/10.1016/j.ultras.2017.07.017.

[20] X.F. Fu, G.Y. Li, M.H. Lu, G. Lu, X. Huang, A 3D space coiling metamaterial with isotropic negative acoustic properties, Appl. Phys. Lett. 111 (2017) 251904. https://doi.org/10.1063/1.5005553.

[21] M. Moleron, M. Serra-Garcia, C. Daraio, Acoustic Fresnel lenses with extraordinary transmission, Appl. Phys. Lett. 105 (2014) 114109. https://doi.org/10.1063/1.4896276.

[22] A.G. Mark, S. Palagi, T. Qiu, P. Fischer, Auxetic metamaterial simplifies soft robot design, in: Proc. - IEEE Int. Conf. Robot. Autom., IEEE, 2016: pp. 4951-4956. https://doi.org/10.1109/ICRA.2016.7487701.

[23] T. Li, X. Hu, Y. Chen, L. Wang, Harnessing out-of-plane deformation to design 3D architected lattice metamaterials with tunable Poisson's ratio, Sci. Rep. 7 (2017) 8949. https://doi.org/10.1038/s41598-017-09218-w.

[24] J. Jiao, The Simulation And Structure Design of Optical Metamaterials, Manchester, 2013.

[25] P. Pa, M.S. Mirotznik, R. McCauley, S. Yarlagadda, K. Duncan, Integrating metamaterials within a structural composite using additive manufacturing methods, IEEE Antennas Propag. Soc. AP-S Int. Symp. (2012) 0-1. 
https://doi.org/10.1109/APS.2012.6349224.

[26] Scopus - Analyze search results, (n.d.).

[27] Y. Liu, X. Zhang, Metamaterials: A new frontier of science and technology, Chem. Soc. Rev. 40 (2011) 2494-2507. https://doi.org/10.1039/c0cs00184h.

[28] K.A. Arpin, A. Mihi, H.T. Johnson, A.J. Baca, J.A. Rogers, J.A. Lewis, P. V. Braun, Multidimensional architectures for functional optical devices, Adv. Mater. 22 (2010) 1084-1101. https://doi.org/10.1002/adma.200904096.

[29] A. Baev, P.N. Prasad, H. Ågren, M. Samoć, M. Wegener, Metaphotonics: An emerging field with opportunities and challenges, Phys. Rep. 594 (2015) 1-60. https://doi.org/10.1016/j.physrep.2015.07.002.

[30] J. Kaschke, M. Wegener, Optical and Infrared Helical Metamaterials, Nanophotonics. 5 (2016) 454-467. https://doi.org/10.1515/nanoph-2016-0005.

[31] S. Guenneau, R. V. Craster, Fundamentals of Acoustic Metamaterials, in: Acoust. Metamaterials SE - 1, Springer, 2013: pp. 1-42. https://doi.org/10.1007/978-94-0074813-2_1.

[32] S.A. Cummer, Transformation acoustics., J. Acoust. Soc. Am. 128 (2010) 2427-2427. https://doi.org/10.1121/1.3508669.

[33] S.A. Cummer, J. Christensen, A. Alù, Controlling sound with acoustic metamaterials, Nat. Rev. Mater. 1 (2016) 16001. https://doi.org/10.1038/natrevmats.2016.1.

[34] J.H. Lee, J.P. Singer, E.L. Thomas, Micro-/nanostructured mechanical metamaterials, Adv. Mater. 24 (2012) 4782-4810. https://doi.org/10.1002/adma.201201644.

[35] H.H. Hilton, D.H. Lee, A.R.A. El Fouly, Generalized viscoelastic designer functionally graded auxetic materials engineered/tailored for specific task performances, Mech. Time-Dependent Mater. 12 (2008) 151-178. https://doi.org/10.1007/s11043-008-9054-9.

[36] A.A. Zadpoor, Mechanical meta-materials, Mater. Horizons. 3 (2016) 371-381. https://doi.org/10.1039/c6mh00065g.

[37] G.N. Greaves, A.L. Greer, R.S. Lakes, T. Rouxel, Poisson's ratio and modern materials, Nat. Mater. 10 (2011) 823-837. https://doi.org/10.1038/nmat3134.

[38] K.K. Saxena, R. Das, E.P. Calius, Three Decades of Auxetics Research - Materials with Negative Poisson's Ratio: A Review, Adv. Eng. Mater. 18 (2016) 1847-1870. https://doi.org/10.1002/adem.201600053.

[39] X. Chen, T.M. Grzegorczyk, B.I. Wu, J. Pacheco, J.A. Kong, Robust method to retrieve the constitutive effective parameters of metamaterials, Phys. Rev. E - Stat. Physics, Plasmas, Fluids, Relat. Interdiscip. Top. 70 (2004) 7. https://doi.org/10.1103/PhysRevE.70.016608.

[40] D.R. Smith, S. Schultz, P. Markoš, C.M. Soukoulis, Determination of effective permittivity and permeability of metamaterials from reflection and transmission coefficients, Phys. Rev. B - Condens. Matter Mater. Phys. 65 (2002) 1-5. https://doi.org/10.1103/PhysRevB.65.195104.

[41] J.B. Pendry, Negative refraction makes a perfect lens, Phys. Rev. Lett. 85 (2000) 3966-3969. https://doi.org/10.1103/PhysRevLett.85.3966.

[42] S.L.N. Ford, Additive Manufacturing Technology: Potential Implications for U.S. Manufacturing Competitiveness., J. Int. Commer. Econ. 6 (2014) 40-75.

https://www.usitc.gov/journals/Vol_VI_Article4_Additive_Manufacturing_Technolog y.pdf.

[43] A. Maddison, Automotive applications, John Wiley \& Sons, 2005. https://doi.org/10.1002/0470014229.ch1.

[44] R.F. Housholder, U.S. Patent 4247508, 1979.

[45] C.R. Garcia, J. Correa, D. Espalin, J.H. Barton, R.C. Rumpf, R. Wicker, V. Gonzalez, 
3D Printing of Anisotropic Metamaterials, Prog. Electromagn. Res. Lett. 34 (2012)

75-82. https://doi.org/10.2528/PIERL12070311.

[46] M. Vaezi, H. Seitz, S. Yang, A review on 3D micro-additive manufacturing technologies, Int. J. Adv. Manuf. Technol. 67 (2013) 1721-1754.

https://doi.org/10.1007/s00170-012-4605-2.

[47] H. Bikas, P. Stavropoulos, G. Chryssolouris, Additive manufacturing methods and modeling approaches: A critical review, Int. J. Adv. Manuf. Technol. 83 (2016) 389405. https://doi.org/10.1007/s00170-015-7576-2.

[48] D. Bourell, J.P. Kruth, M. Leu, G. Levy, D. Rosen, A.M. Beese, A. Clare, Materials for additive manufacturing, CIRP Ann. - Manuf. Technol. 66 (2017) 659-681. https://doi.org/10.1016/j.cirp.2017.05.009.

[49] V.G. Veselago, THE ELECTRODYNAMICS OF SUBSTANCES WITH SIMULTANEOUSLY NEGATIVE VALUES OF $\varepsilon$ AND $\mu$, Sov. Phys. Uspekhi. 10 (1968) 509-514. https://doi.org/10.1070/pu1968v010n04abeh003699.

[50] D.R. Smith, W.J. Padilla, D.C. Vier, S.C. Nemat-Nasser, S. Schultz, Composite medium with simultaneously negative permeability and permittivity, Phys. Rev. Lett. 84 (2000) 4184-4187. https://doi.org/10.1103/PhysRevLett.84.4184.

[51] D. V. Isakov, Q. Lei, F. Castles, C.J. Stevens, C.R.M. Grovenor, P.S. Grant, 3D printed anisotropic dielectric composite with meta-material features, Mater. Des. 93 (2016) 423-430. https://doi.org/10.1016/j.matdes.2015.12.176.

[52] S. Jahani, Z. Jacob, All-dielectric metamaterials, Nat. Nanotechnol. 11 (2016) 23-36. https://doi.org/10.1038/nnano.2015.304.

[53] Metamorphose, (n.d.).

[54] E. Yablonovitch, Photonic Crystals: Semiconductors of Light, Sci. Am. 285 (2009) 46-55. https://doi.org/10.1038/scientificamerican1201-46.

[55] M.J. Colles, Topics in applied physics: Volume 1 - dye lasers, Springer, 2002. https://doi.org/10.1016/0030-3992(74)90069-3.

[56] E. Yablonovitch, Inhibited spontaneous emission in solid-state physics and electronics, Phys. Rev. Lett. 58 (1987) 2059-2062. https://doi.org/10.1103/PhysRevLett.58.2059.

[57] S. John, Strong localization of photons in certain disordered dielectric superlattices, Phys. Rev. Lett. 58 (1987) 2486-2489. https://doi.org/10.1103/PhysRevLett.58.2486.

[58] E. Zbay, G. Tuttle, M. Sigalas, C.M. Soukoulis, K.M. Ho, Defect structures in a layerby-layer photonic band-gap crystal, Phys. Rev. B. 51 (1995) 13961-13965. https://doi.org/10.1103/PhysRevB.51.13961.

[59] Y. Lee, X. Lu, Y. Hao, S. Yang, R. Ubic, J.R.G. Evans, C.G. Parini, Rapid Prototyping of Ceramic Millimeterwave metamaterials: Simulations and Experiments, Microw. Opt. Technol. Lett. 49 (2007) 2090-2093. https://doi.org/10.1002/mop.

[60] X. Lu, Y. Lee, S. Yang, Y. Hao, R. Ubic, J.R.G. Evans, C.G. Parini, Fabrication of electromagnetic crystals by extrusion freeforming, Metamaterials. 2 (2008) 36-44. https://doi.org/10.1016/j.metmat.2007.12.001.

[61] Y. Lee, X. Lu, Y. Hao, S. Yang, J.R.G. Evans, C.G. Parini, Directive millimetrewave antennas using freeformed ceramic metamaterials in planar and cylindrical forms, 2008 IEEE Int. Symp. Antennas Propag. Usn. Natl. Radio Sci. Meet. APSURSI. (2008) 2-5. https://doi.org/10.1109/APS.2008.4619479.

[62] A. Camposeo, L. Persano, M. Farsari, D. Pisignano, Additive Manufacturing: Applications and Directions in Photonics and Optoelectronics, Adv. Opt. Mater. 7 (2019). https://doi.org/10.1002/adom.201800419.

[63] T.F. McNulty, F. Mohammadi, A. Bandyopadhyay, D.J. Shanefield, S.C. Danforth, A. Safari, Development of a binder formulation for fused deposition of ceramics, Rapid Prototyp. J. 4 (1998) 144-150. https://doi.org/10.1108/13552549810239012. 
[64] A. Bellini, L. Shor, S.I. Guceri, New developments in fused deposition modeling of ceramics, Rapid Prototyp. J. 11 (2005) 214-220. https://doi.org/10.1108/13552540510612901.

[65] U. Scheithauer, E. Schwarzer, H.J. Richter, T. Moritz, Thermoplastic 3D printing - An additive manufacturing method for producing dense ceramics, Int. J. Appl. Ceram. Technol. 12 (2015) 26-31. https://doi.org/10.1111/ijac.12306.

[66] U. Scheithauer, A. Bergner, E. Schwarzer, H.J. Richter, T. Moritz, Studies on thermoplastic 3D printing of steel-zirconia composites, J. Mater. Res. 29 (2014) 19311940. https://doi.org/10.1557/jmr.2014.209.

[67] M. Yin, X.Y. Tian, L.L. Wu, D.C. Li, A Broadband and omnidirectional electromagnetic wave concentrator with gradient woodpile structure, Opt. Express. 21 (2013) 19082. https://doi.org/10.1364/oe.21.019082.

[68] S. Zhang, Y. Vardaxoglou, W. Whittow, R. Mittra, 3D-printed flat lens for microwave applications, 2015 Loughbrgh. Antennas Propag. Conf. LAPC 2015. (2015) 1-3. https://doi.org/10.1109/LAPC.2015.7366130.

[69] T. Driscoll, Y. Urzhumov, N. Landy, D. Basov, D.R. Smith, Dielectric metamaterials and composites in the age of 3D printing, and directional cloaking, in: 2013 7th Int. Congr. Adv. Electromagn. Mater. Microwaves Opt. METAMATERIALS 2013, IEEE Computer Society, 2013: pp. 361-363.

https://doi.org/10.1109/MetaMaterials.2013.6809053.

[70] H. Yang, S. Yang, X. Chi, J.R.G. Evans, Fine ceramic lattices prepared by extrusion freeforming, J. Biomed. Mater. Res. - Part B Appl. Biomater. 79 (2006) 116-121. https://doi.org/10.1002/jbm.b.30520.

[71] P.S. Grant, F. Castles, Q. Lei, Y. Wang, J.M. Janurudin, D. Isakov, S. Speller, C. Dancer, C.R.M. Grovenor, Manufacture of electrical and magnetic graded and anisotropic materials for novel manipulations of microwaves, Philos. Trans. R. Soc. A Math. Phys. Eng. Sci. 373 (2015) 20140353. https://doi.org/10.1098/rsta.2014.0353.

[72] J.J. Li, B. Li, Q.M. Peng, J. Zhou, L.T. Li, Fabrication and characterization of directwritten 3D TiO2woodpile electromagnetic bandgap structures, Chinese Phys. B. 23 (2014). https://doi.org/10.1088/1674-1056/23/9/098104.

[73] X.X. Zeng, R. Wang, X.Q. Xi, B. Li, J. Zhou, Terahertz rare-earth orthoferrite metamaterials by 3-D direct writing technology, Opt. Express. 26 (2018) 1705617065. https://doi.org/10.1364/OE.26.017056.

[74] E. Saleh, P. Woolliams, B. Clarke, A. Gregory, S. Greedy, C. Smartt, R. Wildman, I. Ashcroft, R. Hague, P. Dickens, C. Tuck, 3D inkjet-printed UV-curable inks for multifunctional electromagnetic applications, Addit. Manuf. 13 (2017) 143-148. https://doi.org/10.1016/j.addma.2016.10.002.

[75] C.J. Reilly, W.J. Chappell, J.W. Halloran, L.P.B. Katehi, High-frequency electromagnetic bandgap structures via indirect solid freeform fabrication, J. Am. Ceram. Soc. 87 (2004) 1446-1453. https://doi.org/10.1111/j.1551-2916.2004.01446.x.

[76] A. Boltasseva, V.M. Shalaev, Fabrication of optical negative-index metamaterials: Recent advances and outlook, Metamaterials. 2 (2008) 1-17. https://doi.org/10.1016/j.metmat.2008.03.004.

[77] H. Lee, S. Kim, D. De Donno, M.M. Tentzeris, A novel Universal inkjet-printed EBGbacked flexible RFID for rugged on-body and metal mounted applications, in: IEEE MTT-S Int. Microw. Symp. Dig., IEEE, 2012: pp. 1-3. https://doi.org/10.1109/MWSYM.2012.6259728.

[78] S. Kim, Y. Kawahara, A. Georgiadis, A. Collado, M.M. Tentzeris, Low-cost inkjetprinted fully passive RFID tags for calibration-free capacitive/haptic sensor applications, in: IEEE Sens. J., IEEE, 2015: pp. 3135-3145. 
https://doi.org/10.1109/JSEN.2014.2366915.

[79] A. Georgiadis, A. Collado, S. Kim, M.M. Tentzeris, Inkjet-printed meta-material inspired passive antenna sensor for UHF RFID systems, in: 2015 Int. Work. Antenna Technol. IWAT 2015, IEEE, 2015: pp. 92-94. https://doi.org/10.1109/IWAT.2015.7365270.

[80] M. Yoo, H.K. Kim, S. Kim, M. Tentzeris, S. Lim, Silver Nanoparticle-Based InkjetPrinted Metamaterial Absorber on Flexible Paper, IEEE Antennas Wirel. Propag. Lett. 14 (2015) 1718-1721. https://doi.org/10.1109/LAWP.2015.2420712.

[81] D. Lee, H.K. Sung, S. Lim, Flexible subterahertz metamaterial absorber fabrication using inkjet printing technology, Appl. Phys. B Lasers Opt. 122 (2016) 206. https://doi.org/10.1007/s00340-016-6482-0.

[82] H.K. Kim, K. Ling, K. Kim, S. Lim, Flexible inkjet-printed metamaterial absorber for coating a cylindrical object, Opt. Express. 23 (2015) 5898. https://doi.org/10.1364/oe.23.005898.

[83] H.K. Kim, D. Lee, S. Lim, A fluidically tunable metasurface absorber for flexible large-scale wireless ethanol sensor applications, Sensors (Switzerland). 16 (2016) 1246. https://doi.org/10.3390/s16081246.

[84] K. Ling, M. Yoo, W. Su, K. Kim, B. Cook, M.M. Tentzeris, S. Lim, Microfluidic tunable inkjet-printed metamaterial absorber on paper, Opt. Express. 23 (2015) 110120. https://doi.org/10.1364/oe.23.000110.

[85] M. Walther, A. Ortner, H. Meier, U. Löffelmann, P.J. Smith, J.G. Korvink, Terahertz metamaterials fabricated by inkjet printing, Appl. Phys. Lett. 95 (2009) 251107. https://doi.org/10.1063/1.3276544.

[86] K. Murata, J. Matsumoto, A. Tezuka, Y. Matsuba, H. Yokoyama, Super-fine ink-jet printing: Toward the minimal manufacturing system, Microsyst. Technol. 12 (2005) $2-$ 7. https://doi.org/10.1007/s00542-005-0023-9.

[87] K. Takano, T. Kawabata, C.F. Hsieh, K. Akiyama, F. Miyamaru, Y. Abe, Y. Tokuda, R.P. Pan, C.L. Pan, M. Hangyo, Fabrication of terahertz planar metamaterials using a super-fine ink-jet printer, Appl. Phys. Express. 3 (2010) 016701. https://doi.org/10.1143/APEX.3.016701.

[88] K. Kashiwagi, L. Xie, X. Li, T. Kageyama, M. Miura, H. Miyashita, J. Kono, S.S. Lee, Inkjet-printed silver-nanoparticle THz metamaterial, Int. Conf. Infrared, Millimeter, Terahertz Waves, IRMMW-THz. 2016-Novem (2016) 2-3. https://doi.org/10.1109/IRMMW-THz.2016.7758981.

[89] C.N. Bowman, C.J. Kloxin, Toward an enhanced understanding and implementation of photopolymerization reactions, AIChE J. 54 (2008) 2775-2795. https://doi.org/10.1002/aic.11678.

[90] F.P.W. Melchels, J. Feijen, D.W. Grijpma, A review on stereolithography and its applications in biomedical engineering, Biomaterials. 31 (2010) 6121-6130. https://doi.org/10.1016/j.biomaterials.2010.04.050.

[91] M. Farsari, M. Vamvakaki, B.N. Chichkov, Multiphoton polymerization of hybrid materials, J. Opt. 12 (2010) 124001. https://doi.org/10.1088/2040-8978/12/12/124001.

[92] F. Jipa, M. Zamfirescu, A. Velea, M. Popescu, R. Dabu, Femtosecond Laser Lithography in Organic and Non-Organic Materials, Updat. Adv. Lithogr. (2013). https://doi.org/10.5772/56579.

[93] B.H. Cumpston, J.E. Ehrlich, S.M. Kuebler, M. Lipson, S.R. Marder, D. McCordMaughon, J.W. Perry, H. Roeckel, M.C. Rumi, Three-dimensional microfabrication using two-photon polymerization, in: Mater. Device Charact. Micromach., 1998. https://doi.org/https://doi.org/10.1117/12.324052.

[94] B.H. Cumpston, S.P. Ananthavel, S. Barlow, D.L. Dyer, J.E. Ehrlich, L.L. Erskine, 
A.A. Heikal, S.M. Kuebler, I.Y.S. Lee, D. McCord-Maughon, J. Qin, H. Röckel, M. Rumi, X.L. Wu, S.R. Marder, J.W. Perry, Two-photon polymerization initiators for three-dimensional optical data storage and microfabrication, Nature. 398 (1999) 5154. https://doi.org/10.1038/17989.

[95] S. Wu, J. Serbin, M. Gu, Two-photon polymerisation for three-dimensional microfabrication, J. Photochem. Photobiol. A Chem. 181 (2006) 1-11. https://doi.org/10.1016/j.jphotochem.2006.03.004.

[96] D. S., L. De, A.J. G. Otuka, V. Tribuzi, C. R., Two-Photon Polymerization Fabrication of Doped Microstructures, in: A.D.S. Gomes (Ed.), Polymerization, 2012. https://doi.org/10.5772/36061.

[97] K.S. Lee, D.Y. Yang, S.H. Park, R.H. Kim, Recent developments in the use of twophoton polymerization in precise 2D and 3D microfabrications, Polym. Adv. Technol. 17 (2006) 72-82. https://doi.org/10.1002/pat.664.

[98] K.S. Lee, R.H. Kim, D.Y. Yang, S.H. Park, Advances in 3D nano/microfabrication using two-photon initiated polymerization, Prog. Polym. Sci. 33 (2008) 631-681. https://doi.org/10.1016/j.progpolymsci.2008.01.001.

[99] W. Lee, S.A. Pruzinsky, P. V. Braun, Multi-photon polymerization of waveguide structures within 3D photonic crystals, Am. Chem. Soc. Polym. Prepr. Div. Polym. Chem. 43 (2002) 31-32. https://doi.org/10.1002/15214095(20020219)14:4<271::AID-ADMA271>3.3.CO;2-P.

[100] S. Kirihara, Y. Miyamoto, K. Kajiyama, Fabrication of ceramic-polymer photonic crystals by stereolithography and their microwave properties, J. Am. Ceram. Soc. 85 (2002) 1369-1371. https://doi.org/10.1111/j.1151-2916.2002.tb00281.x.

[101] H. Yin, S. Kirihara, Y. Miyamoto, Fabrication of ceramic photonic crystals with diamond structure for microwave applications, J. Am. Ceram. Soc. 87 (2004) 598-601. https://doi.org/10.1111/j.1551-2916.2004.00598.x.

[102] Y.K. Pang, J.C. Lee, C.T. Ho, W.Y. Tam, Realization of woodpile structure using optical interference holography, Opt. Express. 14 (2006) 9013. https://doi.org/10.1364/oe.14.009013.

[103] S. Kirihara, Additive manufacturing of ceramic components using laser scanning stereolithography, Weld. World. 60 (2016) 697-702. https://doi.org/10.1007/s40194016-0331-y.

[104] S. Kirihara, Additive Manufacturing of Micro Functional Structures Through Diameter Variable Laser Stereolithography and Precursor Sintering Heat Treatments, in: Addit. Manuf. Strateg. Technol. Adv. Ceram., John Wiley \& Sons, Inc., Hoboken, NJ, USA, 2016: pp. 1-10. https://doi.org/10.1002/9781119236016.ch1.

[105] S. Kirihara, Stereolithographic Additive Manufacturing of Ceramic Components by Using Nanoparticle Paste Feeding, Mater. Sci. Forum. 879 (2016) 2485-2488. https://doi.org/10.4028/www.scientific.net/msf.879.2485.

[106] S. Kirihara, Stereolithography of ceramic components: fabrication of photonic crystals with diamond structures for terahertz wave modulation, J. Ceram. Soc. Japan. 123 (2015) 816-822. https://doi.org/10.2109/jcersj2.123.816.

[107] W. Chen, S. Kirihara, Y. Miyamoto, Fabrication and measurement of micro threedimensional photonic crystals of $\mathrm{SiO} 2$ ceramic for terahertz wave applications, J. Am. Ceram. Soc. 90 (2007) 2078-2081. https://doi.org/10.1111/j.1551-2916.2007.01676.x.

[108] Q. Liang, D. Li, G. Yang, H. Han, Ultra-wide bandgap of gradient dielectric constant photonic crystal, Mater. Lett. 79 (2012) 48-50. https://doi.org/10.1016/j.matlet.2012.03.082.

[109] M. Yin, X. Yong Tian, H. Xue Han, D. Chen Li, Free-space carpet-cloak based on gradient index photonic crystals in metamaterial regime, Appl. Phys. Lett. 100 (2012) 
124101. https://doi.org/10.1063/1.3696040.

[110] S.M. Rudolph, A. Grbic, A broadband three-dimensional isotropic NRI medium, 2010 IEEE Int. Symp. Antennas Propag. CNC-USNC/URSI Radio Sci. Meet. - Lead. Wave, AP-S/URSI 2010. 1 (2010) 10-13. https://doi.org/10.1109/APS.2010.5561010.

[111] D. Wu, N. Fang, C. Sun, X. Zhang, W.J. Padilla, D.N. Basov, D.R. Smith, S. Schultz, Terahertz plasmonic high pass filter, Appl. Phys. Lett. 83 (2003) 201-203. https://doi.org/10.1063/1.1591083.

[112] J. Serbin, A. Ovsianikov, B. Chichkov, Fabrication of woodpile structures by twophoton polymerization and investigation of their optical properties, Opt. Express. 12 (2004) 5221. https://doi.org/10.1364/opex.12.005221.

[113] W.J. Chen, J.C.W. Lee, J.W. Dong, C.W. Qiu, H.Z. Wang, Fano resonance of threedimensional spiral photonic crystals: Paradoxical transmission and polarization gap, Appl. Phys. Lett. 98 (2011). https://doi.org/10.1063/1.3560338.

[114] M. Thiel, M.S. Rill, G. Von Freymann, M. Wegener, Three-dimensional bi-chiral photonic crystals, Adv. Mater. 21 (2009) 4680-4682.

https://doi.org/10.1002/adma.200901601.

[115] M. Thiel, M. Wegener, G. Von Freymann, Layer-by-layer three-dimensional chiral photonic crystals, 2008 Conf. Quantum Electron. Laser Sci. Conf. Lasers ElectroOptics, CLEO/QELS. 32 (2008) 2547. https://doi.org/10.1109/CLEO.2008.4551903.

[116] M. Straub, M. Gu, Near-infrared photonic crystals with higher-order bandgaps generated by two-photon photopolymerization, Opt. Lett. 27 (2002) 1824. https://doi.org/10.1364/ol.27.001824.

[117] J. Li, B. Jia, M. Gu, Engineering stop gaps of inorganic-organic polymeric 3D woodpile photonic crystals with post-thermal treatment, Opt. Express. 16 (2008) 20073. https://doi.org/10.1364/oe.16.020073.

[118] J.D. Fernandez, J. de Coninck, Multiresolution layered manufacturing, Rapid Prototyp. J. 25 (2019) 87-94. https://doi.org/10.1108/RPJ-07-2017-0146.

[119] S. Wong, M. Deubel, F. Pérez-Willard, S. John, G.A. Ozin, M. Wegener, G. Von Freymann, Direct laser writing of three-dimensional photonic crystals with a complete photonic bandgap in chalcogenide glasses, Adv. Mater. 18 (2006) 265-269. https://doi.org/10.1002/adma.200501973.

[120] L. Li, J.T. Fourkas, Multiphoton polymerization, Mater. Today. 10 (2007) 30-37. https://doi.org/10.1016/S1369-7021(07)70130-X.

[121] C. Lü, B. Yang, High refractive index organic-inorganic nanocomposites: Design, synthesis and application, J. Mater. Chem. 19 (2009) 2884-2901. https://doi.org/10.1039/b816254a.

[122] M. Farsari, A. Ovsianikov, M. Vamvakaki, I. Sakellari, D. Gray, B.N. Chichkov, C. Fotakis, Fabrication of three-dimensional photonic crystal structures containing an active nonlinear optical chromophore, Appl. Phys. A Mater. Sci. Process. 93 (2008) 11-15. https://doi.org/10.1007/s00339-008-4642-8.

[123] J.C.M. Garnett, Colours in Metal Glasses, in Metallic Films, and in Metallic Solutions. II, Philos. Trans. R. Soc. A Math. Phys. Eng. Sci. 205 (1906) 237-288. https://doi.org/10.1098/rsta.1906.0007.

[124] R. Houbertz, P. Declerck, S. Passinger, A. Ovsianikov, J. Serbin, B.N. Chichkov, Investigations on the generation of photonic crystals using two-photon polymerization (2PP) of inorganic-organic hybrid polymers with ultra-short laser pulses, Phys. Status Solidi Appl. Mater. Sci. 204 (2007) 3662-3675. https://doi.org/10.1002/pssa.200776416.

[125] X.M. Duan, H.B. Sun, K. Kaneko, S. Kawata, Two-photon polymerization of metal ions doped acrylate monomers and oligomers for three-dimensional structure 
fabrication, Thin Solid Films. 453-454 (2004) 518-521.

https://doi.org/10.1016/j.tsf.2003.11.126.

[126] M. Gu, B. Jia, J. Li, M.J. Ventura, Fabrication of three-dimensional photonic crystals in quantum-dot-based materials, Laser Photonics Rev. 4 (2010) 414-431. https://doi.org/10.1002/lpor.200910008.

[127] S.Y. Lin, E. Chow, V. Hietala, P.R. Villeneuve, J.D. Joannopoulos, Experimental demonstration of guiding and bending of electromagnetic waves in a photonic crystal, Science (80-. ). 282 (1998) 274-276. https://doi.org/10.1126/science.282.5387.274.

[128] M. Imada, L.H. Lee, M. Okano, S. Kawashima, S. Noda, Development of threedimensional photonic-crystal waveguides at optical-communication wavelengths, Appl. Phys. Lett. 88 (2006) 171107. https://doi.org/10.1063/1.2197942.

[129] S. Noda, K. Tomoda, N. Yamamoto, A. Chutinan, Full three-dimensional photonic bandgap crystals at near-infrared wavelengths, Science (80-. ). 289 (2000) 604-606. https://doi.org/10.1126/science.289.5479.604.

[130] D. Shir, E.C. Nelson, Y.C. Chen, A. Brzezinski, H. Liao, P. V. Braun, P. Wiltzius, K.H.A. Bogart, J.A. Rogers, Three dimensional silicon photonic crystals fabricated by two photon phase mask lithography, Appl. Phys. Lett. 94 (2009) 011101. https://doi.org/10.1063/1.3036955.

[131] D.J. Shir, E.C. Nelson, D. Chanda, A. Brzezinski, P. V. Braun, J.A. Rogers, P. Wiltzius, Dual exposure, two-photon, conformal phase mask lithography for three dimensional silicon inverse woodpile photonic crystals, J. Vac. Sci. Technol. B, Nanotechnol. Microelectron. Mater. Process. Meas. Phenom. 28 (2010) 783-788. https://doi.org/10.1116/1.3456181.

[132] S. Maruo, J.T. Fourkas, Recent progress in multiphoton microfabrication, Laser Photonics Rev. 2 (2008) 100-111. https://doi.org/10.1002/lpor.200710039.

[133] M.S. Rill, C. Plet, M. Thiel, I. Staude, G. Von Freymann, S. Linden, M. Wegener, Photonic metamaterials by direct laser writing and silver chemical vapour deposition, Nat. Mater. 7 (2008) 543-546. https://doi.org/10.1038/nmat2197.

[134] F. Klein, B. Richter, T. Striebel, C.M. Franz, G. Von Freymann, M. Wegener, M. Bastmeyer, Two-component polymer scaffolds for controlled three-dimensional cell culture, Adv. Mater. 23 (2011) 1341-1345. https://doi.org/10.1002/adma.201004060.

[135] S. Rekštyte, E. Kaziulionyte, E. Balčiunas, D. Kaškelyte, M. Malinauskas, Direct laser fabrication of composite material 3D microstructured scaffolds, J. Laser Micro Nanoeng. 9 (2014) 25-30. https://doi.org/10.2961/jlmn.2014.01.0006.

[136] M. Askari, C.J. Tuck, Q. Hu, R.J.M. Hague, R.D. Wildman, Multimaterial manufacture through combining optical tweezers with multiphoton fabrication, J. Laser Micro Nanoeng. 14 (2019) 80-87. https://doi.org/10.2961/jlmn.2019.01.0014.

[137] F. Stellacci, C.A. Bauer, T. Meyer-Friedrichsen, W. Wenseleers, V. Alain, S.M. Kuebler, S.J.K. Pond, Y. Zhang, S.R. Marder, J.W. Perry, Laser and electron-beam induced growth of nanoparticles for 2D and 3D metal patterning, Adv. Mater. 14 (2002) 194-198. https://doi.org/10.1002/1521-4095(20020205)14:3<194::AIDADMA194>3.0.CO;2-W.

[138] E. Blasco, J. Müller, P. Müller, V. Trouillet, M. Schön, T. Scherer, C. BarnerKowollik, M. Wegener, Fabrication of Conductive 3D Gold-Containing Microstructures via Direct Laser Writing, Adv. Mater. 28 (2016) 3592-3595. https://doi.org/10.1002/adma.201506126.

[139] A. Ishikawa, T. Tanaka, S. Kawata, Improvement in the reduction of silver ions in aqueous solution using two-photon sensitive dye, Appl. Phys. Lett. 89 (2006) 79-82. https://doi.org/10.1063/1.2345601.

[140] T. Tanaka, A. Ishikawa, S. Kawata, Two-photon-induced reduction of metal ions for 
fabricating three-dimensional electrically conductive metallic microstructure, Appl. Phys. Lett. 88 (2006) 081107. https://doi.org/10.1063/1.2177636.

[141] C.N. LaFratta, J.T. Fourkas, T. Baldacchini, R.A. Farrer, Multiphoton fabrication, Angew. Chemie - Int. Ed. 46 (2007) 6238-6258.

https://doi.org/10.1002/anie.200603995.

[142] R.P. Chaudhary, G. Ummethala, A. Jaiswal, S. Hawal, S. Saxena, S. Shukla, One-step, subwavelength patterning of plasmonic gratings in metal-polymer composites, RSC Adv. 6 (2016) 113457-113462. https://doi.org/10.1039/c6ra22604c.

[143] S. Shukla, X. Vidal, E.P. Furlani, M.T. Swihart, K.T. Kim, Y.K. Yoon, A. Urbas, P.N. Prasad, Subwavelength direct laser patterning of conductive gold nanostructures by simultaneous photopolymerization and photoreduction, ACS Nano. 5 (2011) 19471957. https://doi.org/10.1021/nn103015g.

[144] F. Formanek, N. Takeyasu, T. Tanaka, K. Chiyoda, A. Ishikawa, S. Kawata, Threedimensional fabrication of metallic nanostructures over large areas by two-photon polymerization, Opt. Express. 14 (2006) 800. https://doi.org/10.1364/opex.14.000800.

[145] V. Mizeikis, S. Juodkazis, R. Tarozaite, J. Juodkazyte, K. Juodkazis, H. Misawa, Fabrication and properties of metalo-dielectric photonic crystal structures for infrared spectral region, Opt. Express. 15 (2007) 8454. https://doi.org/10.1364/oe.15.008454.

[146] X. Xiong, Z.H. Xue, C. Meng, S.C. Jiang, Y.H. Hu, R.W. Peng, M. Wang, Polarization-dependent perfect absorbers/reflectors based on a three-dimensional metamaterial, Phys. Rev. B - Condens. Matter Mater. Phys. 88 (2013) 1-7. https://doi.org/10.1103/PhysRevB.88.115105.

[147] X. Xiong, S.C. Jiang, Y.H. Hu, R.W. Peng, M. Wang, Structured metal film as a perfect absorber, Adv. Mater. 25 (2013) 3994-4000. https://doi.org/10.1002/adma.201300223.

[148] A.I. Aristov, M. Manousidaki, A. Danilov, K. Terzaki, C. Fotakis, M. Farsari, A. V. Kabashin, 3D plasmonic crystal metamaterials for ultra-sensitive biosensing, Sci. Rep. 6 (2016) 25380. https://doi.org/10.1038/srep25380.

[149] M.M. Hossain, M. Gu, Fabrication methods of 3D periodic metallic nano/microstructures for photonics applications, Laser Photonics Rev. 8 (2014) 233249. https://doi.org/10.1002/lpor.201300052.

[150] N. Takeyasu, T. Tanaka, S. Kawata, Fabrication of 3D metal/polymer microstructures by site-selective metal coating, Appl. Phys. A Mater. Sci. Process. 90 (2008) 205-209. https://doi.org/10.1007/s00339-007-4298-9.

[151] Y. Yan, A.A. Bettiol, H. Tanoto, J. Teng, Selective electroless silver plating of three dimensional SU-8 microstructures on silicon for metamaterials applications, Opt. Mater. Express. 1 (2011) 1548-1554. https://doi.org/10.1364/OME.1.001548.

[152] A.I. Kuznetsov, A.B. Evlyukhin, C. Reinhardt, A. Seidel, R. Kiyan, W. Cheng, A. Ovsianikov, B.N. Chichkov, Laser-induced transfer of metallic nanodroplets for plasmonics and metamaterial applications, J. Opt. Soc. Am. B. 26 (2009) B130. https://doi.org/10.1364/josab.26.00b130.

[153] J. Moughames, S. Jradi, T.M. Chan, S. Akil, Y. Battie, A.E. Naciri, Z. Herro, S. Guenneau, S. Enoch, L. Joly, J. Cousin, A. Bruyant, Wavelength-scale light concentrator made by direct 3D laser writing of polymer metamaterials, Sci. Rep. 6 (2016) 33627. https://doi.org/10.1038/srep33627.

[154] J.K. Gansel, M. Thiel, M.S. Rill, M. Decker, K. Bade, V. Saile, G. von Freymann, S. Linden, M. Wegener, Gold Helix Photonic Metamaterial as Broadband Circular Polarizer, Science (80-. ). 325 (2009) 1513-1515. https://doi.org/10.1126/science.1177031.

[155] J. Kaschke, M. Wegener, Gold triple-helix mid-infrared metamaterial by STED- 
inspired laser lithography, Opt. Lett. 40 (2015) 3986.

https://doi.org/10.1364/OL.40.003986.

[156] S.D. Allen, Laser chemical vapor deposition: A technique for selective area deposition, J. Appl. Phys. 52 (1981) 6501-6505. https://doi.org/10.1063/1.328600.

[157] F.T. Wallenberger, Rapid prototyping directly from the vapor phase, Science (80-. ). 267 (1995) 1274-1275. https://doi.org/10.1126/science.267.5202.1274.

[158] M.C. Wanke, O. Lehmann, K. Müller, Q. Wen, M. Stuke, Laser rapid prototyping of photonic band-gap microstructures, Science (80-. ). 275 (1997) 1284-1286. https://doi.org/10.1126/science.275.5304.1284.

[159] C. Oakley, A. Kaur, J.A. Byford, P. Chahal, Aerosol-Jet Printed Quasi-Optical Terahertz Filters, Proc. - Electron. Components Technol. Conf. (2017) 248-253. https://doi.org/10.1109/ECTC.2017.233.

[160] J.Q. Feng, M.J. Renn, Aerosol Jet ${ }^{\circledR}$ Direct-Write for Microscale Additive Manufacturin, J. Micro Nano Manuf. 7 (2019) 011004. https://doi.org/https://doi.org/10.1115/1.4043595.

[161] B. Nagarajan, Z. Hu, X. Song, W. Zhai, J. Wei, Development of Micro Selective Laser Melting: The State of the Art and Future Perspectives, Engineering. 5 (2019) 702-720. https://doi.org/10.1016/j.eng.2019.07.002.

[162] D.M. French, D. Shiffler, High power microwave source with a three dimensional printed metamaterial slow-wave structure, Rev. Sci. Instrum. 87 (2016) 053308. https://doi.org/10.1063/1.4950904.

[163] T. Jaffré, L. Leger, B. Jecko, J. Claus, C. Chaput, Fabrication of 3-D alumina photonic bandgap structures by laser rapid prototyping - Application to the design of three dimensional photonic crystal resonator antenna, in: Conf. Proc. - ICECom 2003 17th Int. Conf. Appl. Electromagn. Commun., IEEE, 2003: pp. 255-258. https://doi.org/10.1109/ICECOM.2003.1291002.

[164] J. Bishop-Moser, C.M. Spadaccini, C. Andres, Metamaterials Manufacturing Pathways to Industrial Competitiveness, Alliance for Manufacturing Foresight Report, 2018. http://hdl.handle.net/2027.42/145155.

[165] S. Pandey, B. Gupta, A. Chanana, A. Nahata, Non-Drude like behaviour of metals in the terahertz spectral range, Adv. Phys. X. 1 (2016) 176-193. https://doi.org/10.1080/23746149.2016.1165079.

[166] S.A. Cummer, Transformation Acoustics, 2013. https://doi.org/10.1007/978-94-0074813-2_8.

[167] V. Fokin, M. Ambati, C. Sun, X. Zhang, Method for retrieving effective properties of locally resonant acoustic metamaterials, Phys. Rev. B - Condens. Matter Mater. Phys. 76 (2007) 144302. https://doi.org/10.1103/PhysRevB.76.144302.

[168] M.R. Haberman, M.D. Guild, Acoustic metamaterials, Phys. Today. 69 (2016) 42-48. https://doi.org/10.1063/PT.3.3198.

[169] G. Ma, P. Sheng, Acoustic metamaterials: From local resonances to broad horizons, Sci. Adv. 2 (2016) e1501595-e1501595. https://doi.org/10.1126/sciadv.1501595.

[170] M.H. Lu, L. Feng, Y.F. Chen, Phononic crystals and acoustic metamaterials, in: Mater. Today, Springer-Verlag, Berlin, 2009: pp. 34-42. https://doi.org/10.1016/S13697021(09)70315-3.

[171] M. Yang, P. Sheng, Sound Absorption Structures: From Porous Media to Acoustic Metamaterials, Annu. Rev. Mater. Res. 47 (2017) 83-114. https://doi.org/10.1146/annurev-matsci-070616-124032.

[172] Y. Wu, M. Yang, P. Sheng, Perspective: Acoustic metamaterials in transition, J. Appl. Phys. 123 (2018) 090901. https://doi.org/10.1063/1.5007682.

[173] S. Yang, J.H. Page, Z. Liu, M.L. Cowan, C.T. Chan, P. Sheng, Focusing of sound in a 
3D phononic crystal, Phys. Rev. Lett. 93 (2004) 024301. https://doi.org/10.1103/PhysRevLett.93.024301.

[174] X. Zhang, Z. Liu, Negative refraction of acoustic waves in two-dimensional phononic crystals, Appl. Phys. Lett. 85 (2004) 341-343. https://doi.org/10.1063/1.1772854.

[175] A. Sukhovich, L. Jing, J.H. Page, Negative refraction and focusing of ultrasound in two-dimensional phononic crystals, Phys. Rev. B - Condens. Matter Mater. Phys. 77 (2008) 014301. https://doi.org/10.1103/PhysRevB.77.014301.

[176] J.H. Page, Focusing of ultrasonic waves by negative refraction in phononic crystals, AIP Adv. 6 (2016) 121606. https://doi.org/10.1063/1.4972204.

[177] H. Su, X. Zhou, X. Xu, G. Hu, Experimental study on acoustic subwavelength imaging of holey-structured metamaterials by resonant tunneling, J. Acoust. Soc. Am. 135 (2014) 1686-1691. https://doi.org/10.1121/1.4868395.

[178] Z. Liang, J. Li, Extreme acoustic metamaterial by coiling up space, Phys. Rev. Lett. 108 (2012) 1-4. https://doi.org/10.1103/PhysRevLett.108.114301.

[179] Z. Liang, T. Feng, S. Lok, F. Liu, K.B. Ng, C.H. Chan, J. Wang, S. Han, S. Lee, J. Li, Space-coiling metamaterials with double negativity and conical dispersion, Sci. Rep. 3 (2013) 1614. https://doi.org/10.1038/srep01614.

[180] Y. Xie, A. Konneker, B.I. Popa, S.A. Cummer, Tapered labyrinthine acoustic metamaterials for broadband impedance matching, Appl. Phys. Lett. 103 (2013) 201906. https://doi.org/10.1063/1.4831770.

[181] J. Kennedy, L. Flanagan, L. Dowling, G.J. Bennett, H. Rice, D. Trimble, The Influence of Additive Manufacturing Processes on the Performance of a Periodic Acoustic Metamaterial, Int. J. Polym. Sci. 2019 (2019) 7029143. https://doi.org/10.1155/2019/7029143.

[182] M.H. Lu, L. Feng, Y.F. Chen, Phononic crystals and acoustic metamaterials, in: Mater. Today, Springer-Verlag, Berlin, 2009: pp. 34-42. https://doi.org/10.1016/S13697021(09)70315-3.

[183] J.H. Page, Focusing of ultrasonic waves by negative refraction in phononic crystals, AIP Adv. 6 (2016). https://doi.org/10.1063/1.4972204.

[184] M.H. Lu, L. Feng, Y.F. Chen, Phononic crystals and acoustic metamaterials, Mater. Today. 12 (2009) 34-42. https://doi.org/10.1016/S1369-7021(09)70315-3.

[185] M. Miniaci, A. Marzani, N. Testoni, L. De Marchi, Complete band gaps in a polyvinyl chloride (PVC) phononic plate with cross-like holes: Numerical design and experimental verification, Ultrasonics. 56 (2015) 251-259. https://doi.org/10.1016/j.ultras.2014.07.016.

[186] O. Yuksel, C. Yilmaz, Shape optimization of phononic band gap structures incorporating inertial amplification mechanisms, J. Sound Vib. 355 (2015) 232-245. https://doi.org/10.1016/j.jsv.2015.06.016.

[187] F. Lucklum, M. Vellekoop, Design and Fabrication Challenges for Millimeter-Scale Three-Dimensional Phononic Crystals, Crystals. 7 (2017) 348. https://doi.org/10.3390/cryst7110348.

[188] K.H. Matlack, A. Bauhofer, S. Krödel, A. Palermo, C. Daraio, Composite 3D-printed metastructures for low-frequency and broadband vibration absorption, Proc. Natl. Acad. Sci. 113 (2016) 8386-8390. https://doi.org/10.1073/pnas.1600171113.

[189] T. Delpero, S. Schoenwald, A. Zemp, A. Bergamini, Structural engineering of threedimensional phononic crystals, J. Sound Vib. 363 (2016) 156-165. https://doi.org/10.1016/j.jsv.2015.10.033.

[190] L. D’Alessandro, E. Belloni, R. Ardito, A. Corigliano, F. Braghin, Modeling and experimental verification of an ultra-wide bandgap in 3D phononic crystal, Appl. Phys. Lett. 109 (2016) 221907. https://doi.org/10.1063/1.4971290. 
[191] X.H. Zhang, Z.G. Qu, X.C. He, D.L. Lu, Experimental study on the sound absorption characteristics of continuously graded phononic crystals, AIP Adv. 6 (2016) 105205. https://doi.org/10.1063/1.4965923.

[192] F. Lucklum, M.J. Vellekoop, Rapid prototyping of 3D phononic crystals using highresolution stereolithography fabrication, Procedia Eng. 120 (2015) 1095-1098. https://doi.org/10.1016/j.proeng.2015.08.783.

[193] F. Lucklum, M.J. Vellekoop, Realization of Complex 3-D Phononic Crystals with Wide Complete Acoustic Band Gaps, IEEE Trans. Ultrason. Ferroelectr. Freq. Control. 63 (2016) 796-797. https://doi.org/10.1109/TUFFC.2016.2543527.

[194] Y. Lai, Z.Q. Zhang, Large band gaps in elastic phononic crystals with air inclusions, Appl. Phys. Lett. 83 (2003) 3900-3902. https://doi.org/10.1063/1.1625998.

[195] S. Laureti, D.A. Hutchins, L.A.J. Davis, S.J. Leigh, M. Ricci, High-resolution acoustic imaging at low frequencies using 3D-printed metamaterials, AIP Adv. 6 (2016) 121701. https://doi.org/10.1063/1.4968606.

[196] K. Cai, B. Román-Manso, J.E. Smay, J. Zhou, M.I. Osendi, M. Belmonte, P. Miranzo, Geometrically complex silicon carbide structures fabricated by robocasting, J. Am. Ceram. Soc. 95 (2012) 2660-2666. https://doi.org/10.1111/j.1551-2916.2012.05276.x.

[197] A. Kruisová, M. Ševčík, H. Seiner, P. Sedlák, B. Román-Manso, P. Miranzo, M. Belmonte, M. Landa, Ultrasonic bandgaps in 3D-printed periodic ceramic microlattices, Ultrasonics. 82 (2018) 91-100. https://doi.org/10.1016/j.ultras.2017.07.017.

[198] A. Kruisová, H. Seiner, P. Sedlák, M. Landa, B. Román-Manso, P. Miranzo, M. Belmonte, Acoustic metamaterial behavior of three-dimensional periodic architectures assembled by robocasting, Appl. Phys. Lett. 105 (2014) 211904. https://doi.org/10.1063/1.4902810.

[199] F. Warmuth, C. Körner, Phononic band gaps in 2D quadratic and 3D cubic cellular structures, Materials (Basel). 8 (2015) 8327-8337. https://doi.org/10.3390/ma8125463.

[200] M. Wormser, F. Warmuth, C. Körner, Evolution of full phononic band gaps in periodic cellular structures, Appl. Phys. A Mater. Sci. Process. 123 (2017) 1-11. https://doi.org/10.1007/s00339-017-1278-6.

[201] M. Wormser, F. Wein, M. Stingl, C. Körner, Design and additive manufacturing of 3D phononic band gap structures based on gradient based optimization, Materials (Basel). 10 (2017) 1125. https://doi.org/10.3390/ma10101125.

[202] R. Schittny, T. Bückmann, M. Kadic, M. Wegener, Elastic measurements on macroscopic three-dimensional pentamode metamaterials, Appl. Phys. Lett. 103 (2013) 231905. https://doi.org/10.1063/1.4838663.

[203] R. Hedayati, A.M. Leeflang, A.A. Zadpoor, Additively manufactured metallic pentamode meta-materials, Appl. Phys. Lett. 110 (2017) 091905. https://doi.org/10.1063/1.4977561.

[204] K. Yu, N.X. Fang, G. Huang, Q. Wang, Magnetoactive Acoustic Metamaterials, Adv. Mater. 30 (2018) 1706348. https://doi.org/10.1002/adma.201706348.

[205] M. Badreddine Assouar, M. Senesi, M. Oudich, M. Ruzzene, Z. Hou, Broadband platetype acoustic metamaterial for low-frequency sound attenuation, Appl. Phys. Lett. 101 (2012) 173505. https://doi.org/10.1063/1.4764072.

[206] M. Rupin, F. Lemoult, G. Lerosey, P. Roux, Experimental demonstration of ordered and disordered multiresonant metamaterials for lamb waves, Phys. Rev. Lett. 112 (2014) 234301. https://doi.org/10.1103/PhysRevLett.112.234301.

[207] A. Colombi, P. Roux, S. Guenneau, P. Gueguen, R. V. Craster, Forests as a natural seismic metamaterial: Rayleigh wave bandgaps induced by local resonances, Sci. Rep. 6 (2016) 19238. https://doi.org/10.1038/srep19238. 
[208] O.R. Bilal, A. Foehr, C. Daraio, Observation of trampoline phenomena in 3D-printed metamaterial plates, Extrem. Mech. Lett. 15 (2017) 103-107. https://doi.org/10.1016/j.eml.2017.06.004.

[209] A. Elayouch, M. Addouche, A. Khelif, Extensive tailorability of sound absorption using acoustic metamaterials, J. Appl. Phys. 124 (2018) 155103. https://doi.org/10.1063/1.5035129.

[210] C. Casarini, B. Tiller, C. Mineo, C.N. MacLeod, J.F.C. Windmill, J.C. Jackson, Enhancing the Sound Absorption of Small-Scale 3-D Printed Acoustic Metamaterials Based on Helmholtz Resonators, IEEE Sens. J. 18 (2018) 7949-7955. https://doi.org/10.1109/JSEN.2018.2865129.

[211] M. Yang, P. Sheng, An Integration Strategy for Acoustic Metamaterials to Achieve Absorption by Design, Appl. Sci. 8 (2018) 1247. https://doi.org/10.3390/app8081247.

[212] R. Ghaffarivardavagh, J. Nikolajczyk, R. Glynn Holt, S. Anderson, X. Zhang, Hornlike space-coiling metamaterials toward simultaneous phase and amplitude modulation, Nat. Commun. 9 (2018) 1349. https://doi.org/10.1038/s41467-018-03839$\mathrm{z}$.

[213] W. Wang, Y. Xie, A. Konneker, B.I. Popa, S.A. Cummer, Design and demonstration of broadband thin planar diffractive acoustic lenses, Appl. Phys. Lett. 105 (2014) 101904. https://doi.org/10.1063/1.4895619.

[214] G. Memoli, M. Caleap, M. Asakawa, D.R. Sahoo, B.W. Drinkwater, S. Subramanian, Metamaterial bricks and quantization of meta-surfaces, Nat. Commun. 8 (2017) 14608. https://doi.org/10.1038/ncomms14608.

[215] S.K. Maurya, A. Pandey, S. Shukla, S. Saxena, Predicting double negativity using transmitted phase in space coiling metamaterials, R. Soc. Open Sci. 5 (2018) 33683. https://doi.org/10.1098/rsos.171042.

[216] W. Wang, Y. Xie, B.I. Popa, S.A. Cummer, Subwavelength diffractive acoustics and wavefront manipulation with a reflective acoustic metasurface, J. Appl. Phys. 120 (2016) 5553. https://doi.org/10.1063/1.4967738.

[217] Y. Xie, B.I. Popa, L. Zigoneanu, S.A. Cummer, Measurement of a broadband negative index with space-coiling acoustic metamaterials, Phys. Rev. Lett. 110 (2013) 175501. https://doi.org/10.1103/PhysRevLett.110.175501.

[218] Z. Jia, J. Li, C. Shen, Y. Xie, S.A. Cummer, Systematic design of broadband pathcoiling acoustic metamaterials, J. Appl. Phys. 123 (2018) 025101. https://doi.org/10.1063/1.5009488.

[219] X. Zhu, K. Li, P. Zhang, J. Zhu, J. Zhang, C. Tian, S. Liu, Implementation of dispersion-free slow acoustic wave propagation and phase engineering with helicalstructured metamaterials, Nat. Commun. 7 (2016) 11731. https://doi.org/10.1038/ncomms11731.

[220] Y. Ding, E.C. Statharas, K. Yao, M. Hong, A broadband acoustic metamaterial with impedance matching layer of gradient index, Appl. Phys. Lett. 110 (2017) 241903. https://doi.org/10.1063/1.4986472.

[221] Y. Li, X. Jiang, R.Q. Li, B. Liang, X.Y. Zou, L.L. Yin, J.C. Cheng, Experimental realization of full control of reflected waves with subwavelength acoustic metasurfaces, Phys. Rev. Appl. 2 (2014) 064002. https://doi.org/10.1103/PhysRevApplied.2.064002.

[222] K. Tang, C. Qiu, M. Ke, J. Lu, Y. Ye, Z. Liu, Anomalous refraction of airborne sound through ultrathin metasurfaces, Sci. Rep. 4 (2014) 6517. https://doi.org/10.1038/srep06517.

[223] T. Frenzel, M. Kadic, M. Wegener, Three-dimensional mechanical metamaterials with a twist, Science (80-. ). 358 (2017) 1072-1074. 
https://doi.org/10.1126/science.aao4640.

[224] X. Jiang, B. Liang, X.Y. Zou, L.L. Yin, J.C. Cheng, Broadband field rotator based on acoustic metamaterials, Appl. Phys. Lett. 104 (2014) 083510. https://doi.org/10.1063/1.4866333.

[225] Y. Xie, Y. Fu, Z. Jia, J. Li, C. Shen, Y. Xu, H. Chen, S.A. Cummer, Acoustic Imaging with Metamaterial Luneburg Lenses, Sci. Rep. 8 (2018) 16188. https://doi.org/10.1038/s41598-018-34581-7.

[226] A. Climente, D. Torrent, J. Sánchez-Dehesa, Sound focusing by gradient index sonic lenses, Appl. Phys. Lett. 97 (2010) 104103. https://doi.org/10.1063/1.3488349.

[227] W. Lee, D.Y. Kang, J. Song, J.H. Moon, D. Kim, Controlled Unusual Stiffness of Mechanical Metamaterials, Sci. Rep. 6 (2016) 20312. https://doi.org/10.1038/srep20312.

[228] Francesco dell'Isola, Pierre Seppecher, Jean Jacques Alibert, Tomasz Lekszycki, Roman Grygoruk, Marek Pawlikowski, David Steigmann, Ivan Giorgio, Ugo Andreaus, Emilio Turco, Maciej Gołaszewski, Nicola Rizzi, Claude Boutin, Victor A. Eremeyev, Anil Misra, Luca Placidi, Emilio Barchiesi, Leopoldo Greco, Massimo Cuomo, Antonio Cazzani, Alessandro Della Corte, Antonio Battista, Daria Scerrato, Inna Zurba Eremeeva, Yosra Rahali, Jean-François Ganghoffer, Wolfgang Müller, Gregor Ganzosch, Mario Spagnuolo, Aron Pfaff, Katarzyna Barcz, Klaus Hoschke, Jan Neggers \& François Hild: Pantographic metamaterials: an example of mathematically driven design and of its technological challenges Continuum Mechanics and Thermodynamics volume 31, pages851-884(2019). https://doi.org/10.1007/s00161018-0689-8

[229] J. Bauer, L.R. Meza, T.A. Schaedler, R. Schwaiger, X. Zheng, L. Valdevit, Nanolattices: An Emerging Class of Mechanical Metamaterials, Adv. Mater. 29 (2017) 1701850. https://doi.org/10.1002/adma.201701850.

[230] A. Clausen, F. Wang, J.S. Jensen, O. Sigmund, J.A. Lewis, Topology Optimized Architectures with Programmable Poisson's Ratio over Large Deformations, Adv. Mater. 27 (2015) 5523-5527. https://doi.org/10.1002/adma.201502485.

[231] E. Andreassen, B.S. Lazarov, O. Sigmund, Design of manufacturable 3D extremal elastic microstructure, Mech. Mater. 69 (2014) 1-10. https://doi.org/10.1016/j.mechmat.2013.09.018.

[232] L. Yang, O. Harrysson, H. West, D. Cormier, Mechanical properties of 3D re-entrant honeycomb auxetic structures realized via additive manufacturing, Int. J. Solids Struct. 69-70 (2015) 475-490. https://doi.org/10.1016/j.ijsolstr.2015.05.005.

[233] M.N. Ali, J.J.C. Busfield, I.U. Rehman, Auxetic oesophageal stents: Structure and mechanical properties, J. Mater. Sci. Mater. Med. 25 (2014) 527-553. https://doi.org/10.1007/s10856-013-5067-2.

[234] T. Bückmann, R. Schittny, M. Thiel, M. Kadic, G.W. Milton, M. Wegener, On threedimensional dilational elastic metamaterials, New J. Phys. 16 (2014) 033032. https://doi.org/10.1088/1367-2630/16/3/033032.

[235] F. Warmuth, F. Osmanlic, L. Adler, M.A. Lodes, C. Körner, Fabrication and characterisation of a fully auxetic 3D lattice structure via selective electron beam melting, Smart Mater. Struct. 26 (2017) 025013. https://doi.org/10.1088/1361$665 X / 26 / 2 / 025013$

[236] J. Schwerdtfeger, F. Schury, M. Stingl, F. Wein, R.F. Singer, C. Körner, Mechanical characterisation of a periodic auxetic structure produced by SEBM, Phys. Status Solidi Basic Res. 249 (2012) 1347-1352. https://doi.org/10.1002/pssb.201084211.

[237] J. Schwerdtfeger, F. Wein, G. Leugering, R.F. Singer, C. Körner, M. Stingl, F. Schury, Design of auxetic structures via mathematical optimization, Adv. Mater. 23 (2011) 
2650-2654. https://doi.org/10.1002/adma.201004090.

[238] R. Critchley, I. Corni, J.A. Wharton, F.C. Walsh, R.J.K. Wood, K.R. Stokes, The preparation of auxetic foams by three-dimensional printing and their characteristics, Adv. Eng. Mater. 15 (2013) 980-985. https://doi.org/10.1002/adem.201300030.

[239] R. Hedayati, A.M. Leeflang, A.A. Zadpoor, Additively manufactured metallic pentamode meta-materials, Appl. Phys. Lett. 110 (2017) 091905. https://doi.org/10.1063/1.4977561.

[240] T.A. Schaedler, A.J. Jacobsen, A. Torrents, A.E. Sorensen, J. Lian, J.R. Greer, L. Valdevit, W.B. Carter, Ultralight metallic microlattices, Science (80-. ). 334 (2011) 962-965. https://doi.org/10.1126/science.1211649.

[241] P. Pour Shahid Saeed Abadi, S.B. Hutchens, J.R. Greer, B.A. Cola, S. Graham, Buckling-driven delamination of carbon nanotube forests, Appl. Phys. Lett. 102 (2013) 223103. https://doi.org/10.1063/1.4802080.

[242] K. Bertoldi, V. Vitelli, J. Christensen, M. Van Hecke, Flexible mechanical metamaterials, Nat. Rev. Mater. (2017). https://doi.org/10.1038/natrevmats.2017.66.

[243] H.M.A. Kolken, A.A. Zadpoor, Auxetic mechanical metamaterials, RSC Adv. 7 (2017) 5111-5129. https://doi.org/10.1039/c6ra27333e.

[244] A.N. Norris, Acoustic metafluids, J. Acoust. Soc. Am. 125 (2009) 839-849. https://doi.org/10.1121/1.3050288.

[245] I. Mazínová, P. Florian, Materials selection in mechanical design, Lect. Notes Mech. Eng. 16 (2014) 145-153. https://doi.org/10.1007/978-3-319-05203-8_21.

[246] X. Zheng, H. Lee, T.H. Weisgraber, M. Shusteff, J. DeOtte, E.B. Duoss, J.D. Kuntz, M.M. Biener, Q. Ge, J.A. Jackson, S.O. Kucheyev, N.X. Fang, C.M. Spadaccini, Ultralight, ultrastiff mechanical metamaterials, Science (80-. ). 344 (2014) 1373-1377. https://doi.org/10.1126/science.1252291.

[247] T. Bückmann, M. Thiel, M. Kadic, R. Schittny, M. Wegener, An elasto-mechanical unfeelability cloak made of pentamode metamaterials, Nat. Commun. 5 (2014) 4130. https://doi.org/10.1038/ncomms5130.

[248] S. Hengsbach, A.D. Lantada, Direct laser writing of auxetic structures: Present capabilities and challenges, Smart Mater. Struct. 23 (2014) 085033. https://doi.org/10.1088/0964-1726/23/8/085033.

[249] T. Bückmann, N. Stenger, M. Kadic, J. Kaschke, A. Frölich, T. Kennerknecht, C. Eberl, M. Thiel, M. Wegener, Tailored 3D mechanical metamaterials made by dip-in direct-laser-writing optical lithography, Adv. Mater. 24 (2012) 2710-2714. https://doi.org/10.1002/adma.201200584.

[250] M. Kadic, T. Bückmann, R. Schittny, P. Gumbsch, M. Wegener, Pentamode metamaterials with independently tailored bulk modulus and mass density, Phys. Rev. Appl. 2 (2014) 054007. https://doi.org/10.1103/PhysRevApplied.2.054007.

[251] Q. Wang, J.A. Jackson, Q. Ge, J.B. Hopkins, C.M. Spadaccini, N.X. Fang, Lightweight Mechanical Metamaterials with Tunable Negative Thermal Expansion, Phys. Rev. Lett. 117 (2016) 175901. https://doi.org/10.1103/PhysRevLett.117.175901.

[252] M. Mohsenizadeh, F. Gasbarri, M. Munther, A. Beheshti, K. Davami, Additivelymanufactured lightweight Metamaterials for energy absorption, Mater. Des. 139 (2018) 521-530. https://doi.org/10.1016/j.matdes.2017.11.037.

[253] A.J. Jacobsen, J.A. Kolodziejska, R. Doty, K.D. Fink, C. Zhou, C.S. Roper, W.B. Carter, Interconnected self-propagating photopolymer waveguides: An alternative to stereolithography for rapid formation of lattice-based open-cellular materials, in: Int. Solid Free. Fabr. Symp., 2010: pp. 846-853.

[254] A.J. Jacobsen, S. Mahoney, W.B. Carter, S. Nutt, Vitreous carbon micro-lattice structures, Carbon N. Y. 49 (2011) 1025-1032. 
https://doi.org/10.1016/j.carbon.2010.10.059.

[255] Z.C. Eckel, C. Zhou, J.H. Martin, A.J. Jacobsen, W.B. Carter, T.A. Schaedler, Additive manufacturing of polymer-derived ceramics, Science (80-. ). 351 (2016) 5862. https://doi.org/10.1126/science.aad2688.

[256] J. Bauer, A. Schroer, R. Schwaiger, O. Kraft, Approaching theoretical strength in glassy carbon nanolattices, Nat. Mater. 15 (2016) 438-443. https://doi.org/10.1038/nmat4561.

[257] A. Torrents, T.A. Schaedler, A.J. Jacobsen, W.B. Carter, L. Valdevit, Characterization of nickel-based microlattice materials with structural hierarchy from the nanometer to the millimeter scale, Acta Mater. 60 (2012) 3511-3523. https://doi.org/10.1016/j.actamat.2012.03.007.

[258] L. Valdevit, S.W. Godfrey, T.A. Schaedler, A.J. Jacobsen, W.B. Carter, Compressive strength of hollow microlattices: Experimental characterization, modeling, and optimal design, J. Mater. Res. 28 (2013) 2461-2473. https://doi.org/10.1557/jmr.2013.160.

[259] S.C. Han, J.W. Lee, K. Kang, A New Type of Low Density Material: Shellular, Adv. Mater. 27 (2015) 5506-5511. https://doi.org/10.1002/adma.201501546.

[260] M. Mieszala, M. Hasegawa, G. Guillonneau, J. Bauer, R. Raghavan, C. Frantz, O. Kraft, S. Mischler, J. Michler, L. Philippe, Micromechanics of Amorphous Metal/Polymer Hybrid Structures with 3D Cellular Architectures: Size Effects, Buckling Behavior, and Energy Absorption Capability, Small. 13 (2017) 1602514. https://doi.org/10.1002/smll.201602514.

[261] X. Wendy Gu, J.R. Greer, Ultra-strong architected Cu meso-lattices, Extrem. Mech. Lett. 2 (2015) 7-14. https://doi.org/10.1016/j.eml.2015.01.006.

[262] L.C. Montemayor, J.R. Greer, Mechanical Response of Hollow Metallic Nanolattices: Combining Structural and Material Size Effects, J. Appl. Mech. 82 (2015) 071012. https://doi.org/10.1115/1.4030361.

[263] L.R. Meza, S. Das, J.R. Greer, Strong, lightweight, and recoverable three-dimensional ceramic nanolattices, Science (80-. ). 345 (2014) 1322-1326. https://doi.org/10.1126/science.1255908.

[264] J. Bauer, S. Hengsbach, I. Tesari, R. Schwaiger, O. Kraft, High-strength cellular ceramic composites with 3D microarchitecture, Proc. Natl. Acad. Sci. 111 (2014) 2453-2458. https://doi.org/10.1073/pnas.1315147111.

[265] L.R. Meza, A.J. Zelhofer, N. Clarke, A.J. Mateos, D.M. Kochmann, J.R. Greer, Resilient 3D hierarchical architected metamaterials, Proc. Natl. Acad. Sci. 112 (2015) 11502-11507. https://doi.org/10.1073/pnas.1509120112.

[266] X. Zheng, W. Smith, J. Jackson, B. Moran, H. Cui, D. Chen, J. Ye, N. Fang, N. Rodriguez, T. Weisgraber, C.M. Spadaccini, Multiscale metallic metamaterials, Nat. Mater. 15 (2016) 1100-1106. https://doi.org/10.1038/nmat4694.

[267] L.R. Meza, J.R. Greer, Mechanical characterization of hollow ceramic nanolattices, J. Mater. Sci. 49 (2014) 2496-2508. https://doi.org/10.1007/s10853-013-7945-x.

[268] Y. Jiang, Q. Wang, Highly-stretchable 3D-architected Mechanical Metamaterials, Sci. Rep. 6 (2016) 34147. https://doi.org/10.1038/srep34147.

[269] R. Wildman, I. Ashcroft, M. Abdi, Design optimization for an additively manufactured automotive component, Int. J. Powertrains. 7 (2017) 142-161. https://doi.org/10.1504/ijpt.2018.10009559.

[270] S. Amin Yavari, S.M. Ahmadi, R. Wauthle, B. Pouran, J. Schrooten, H. Weinans, A.A. Zadpoor, Relationship between unit cell type and porosity and the fatigue behavior of selective laser melted meta-biomaterials, J. Mech. Behav. Biomed. Mater. 43 (2015) 91-100. https://doi.org/10.1016/j.jmbbm.2014.12.015.

[271] S. Li, H. Hassanin, M.M. Attallah, N.J.E. Adkins, K. Essa, The development of TiNi- 
based negative Poisson's ratio structure using selective laser melting, Acta Mater. 105 (2016) 75-83. https://doi.org/10.1016/j.actamat.2015.12.017.

[272] C. Bonatti, D. Mohr, Large deformation response of additively-manufactured FCC metamaterials: From octet truss lattices towards continuous shell mesostructures, Int. J. Plast. 92 (2017) 122-147. https://doi.org/10.1016/j.ijplas.2017.02.003.

[273] L. Yang, D. Cormier, H. West, O. Harrysson, K. Knowlson, Non-stochastic Ti-6Al-4V foam structures with negative Poisson's ratio, Mater. Sci. Eng. A. 558 (2012) 579-585. https://doi.org/10.1016/j.msea.2012.08.053.

[274] Y. Chen, T. Li, F. Scarpa, L. Wang, Lattice Metamaterials with Mechanically Tunable Poisson's Ratio for Vibration Control, Phys. Rev. Appl. 7 (2017) 024012. https://doi.org/10.1103/PhysRevApplied.7.024012.

[275] K. Wang, Y.H. Chang, Y.W. Chen, C. Zhang, B. Wang, Designable dual-material auxetic metamaterials using three-dimensional printing, Mater. Des. 67 (2015) 159164. https://doi.org/10.1016/j.matdes.2014.11.033.

[276] S. Yuan, F. Shen, J. Bai, C.K. Chua, J. Wei, K. Zhou, 3D soft auxetic lattice structures fabricated by selective laser sintering: TPU powder evaluation and process optimization, Mater. Des. 120 (2017) 317-327. https://doi.org/10.1016/j.matdes.2017.01.098.

[277] M. Bodaghi, A.R. Damanpack, G.F. Hu, W.H. Liao, Large deformations of soft metamaterials fabricated by 3D printing, Mater. Des. 131 (2017) 81-91. https://doi.org/10.1016/j.matdes.2017.06.002.

[278] A. Ion, J. Frohnhofen, L. Wall, R. Kovacs, M. Alistar, J. Lindsay, P. Lopes, H.-T. Chen, P. Baudisch, Metamaterial Mechanisms, in: Proc. 29th Annu. Symp. User Interface Softw. Technol. - UIST '16, 2016: pp. 529-539. https://doi.org/10.1145/2984511.2984540.

[279] H. Yazdani Sarvestani, A.H. Akbarzadeh, A. Mirbolghasemi, K. Hermenean, 3D printed meta-sandwich structures: Failure mechanism, energy absorption and multi-hit capability, Mater. Des. 160 (2018) 179-193. https://doi.org/10.1016/j.matdes.2018.08.061.

[280] A. Rafsanjani and D. Pasini, Bistable Auxetic Mechanical Metamaterials Inspired by Ancient Geometric Motifs", Extreme Mech. Lett. 9 (2016) 291-296.

[281] S. Kamrava, D. Mousanezhad, H. Ebrahimi, R. Ghosh and A. Vaziri, Origami-based cellular metamaterial with auxetic, bistable, and self-locking properties, Scient. Rep. 7 (2017) 46046

.[282] A. Rafsanjani, A.H. Akbarzadeh and D. Pasini, Snapping Mechanical Metamaterials under Tension, Adv. Mat. 27 (2015) 5931-5935.

[283] K. Che, C. Yuan, J. Wu, H.J. Qi and J. Meaud, Three-dimensional-printed multistable mechanical metamaterials with a deterministic deformation sequence", J. Appl. Mech. 84 (2017) 011004.

[284] H. Yang and L. Ma, Angle-dependent transitions between structural bistability and multistability, Adv. Eng. Mat. 22 (2020) 1900871.

[285] S. Sengupta and S. Li, Harnessing the anisotropic multistability of stacked-origami mechanical metamaterials for effective modulus programming, J. Intell. Mat. Syst. and Struct. 29 (2018) 2933-2945.

[286] H. Fang, S. Li, Ji H and K.W. Wang, Dynamics of a bistable Miura-origami structure. Phys. Rev. E 95 (2017) 52211.

[287] Z. Vangelatos, A. Micheletti, C.P. Grigoropoulos and F. Fraternali, Design and testing of bistable lattices with tensegrity architecture and nanoscale features fabricated by multiphoton lithography. Nanomaterials 10 (2020) 652.

[288] H. Y. Jeong, S. C. An, I. C. Seo, E. Lee, S. Ha, N. Kim and Y. C Jun, 3D printing of 
twisting and rotational bistable structures with tuning elements. Scientific reports 9 (2019) $1-9$.

[289] M. Rafiee, R. D. Farahani, and D. Therriault, "Multi-Material 3D and 4D Printing: A Survey," Adv. Sci., vol. 7, no. 12, p. 1902307, Jun. 2020.

[290] L. Yan, Y. Chen, and F. Liou, "Additive manufacturing of functionally graded metallic materials using laser metal deposition," Addit. Manuf., vol. 31, p. 100901, Jan. 2020.

[291] C. Wei, L. Li, X. Zhang, and Y. H. Chueh, "3D printing of multiple metallic materials via modified selective laser melting," CIRP Ann., vol. 67, no. 1, pp. 245-248, Jan. 2018.

[292] K. Wang, Y. H. Chang, Y. W. Chen, C. Zhang, and B. Wang, "Designable dualmaterial auxetic metamaterials using three-dimensional printing," Mater. Des., vol. 67, pp. 159-164, Feb. 2015.

[293] K. Wang et al., "Controlling the mechanical behavior of dual-material 3D printed meta-materials for patient-specific tissue-mimicking phantoms," Mater. Des., vol. 90, pp. 704-712, Jan. 2016.

[294] K. Wang, C. Wu, Z. Qian, C. Zhang, B. Wang, and M. A. Vannan, "Dual-material 3D printed metamaterials with tunable mechanical properties for patient-specific tissuemimicking phantoms," Addit. Manuf., vol. 12, pp. 31-37, Oct. 2016.

[295] P. Sitthi-Amorn et al., "MultiFab: A machine vision assisted platform for multimaterial 3D printing," in ACM Transactions on Graphics, 2015, vol. 34, no. 4, pp. 111.

[296] M. J. Mirzaali, A. Caracciolo, H. Pahlavani, S. Janbaz, L. Vergani, and A. A. Zadpoor, "Multi-material 3D printed mechanical metamaterials: Rational design of elastic properties through spatial distribution of hard and soft phases," Appl. Phys. Lett., vol. 113, no. 24, p. 241903, Dec. 2018.

[297] K. K. Saxena, R. Das, and E. P. Calius, "3D printable multimaterial cellular auxetics with tunable stiffness," Jul. 2017.

[298] Y. Jiang and Y. Li, "3D Printed Auxetic Mechanical Metamaterial with Chiral Cells and Re-entrant Cores," Sci. Rep., vol. 8, no. 1, p. 2397, Dec. 2018.

[299] L. Wang, J. Lau, E. L. Thomas, and M. C. Boyce, "Co-continuous composite materials for stiffness, strength, and energy dissipation,” Adv. Mater., vol. 23, no. 13, pp. 1524-1529, Apr. 2011.

[300] E. Saleh et al., "3D inkjet-printed UV-curable inks for multi-functional electromagnetic applications," Addit. Manuf., vol. 13, pp. 143-148, Jan. 2017.

[301] P. Vogiatzis, S. Chen, X. Wang, T. Li, and L. Wang, "Topology optimization of multi-material negative Poisson's ratio metamaterials using a reconciled level set method," CAD Comput. Aided Des., vol. 83, pp. 15-32, Feb. 2017.

[302] D. V. Isakov, Q. Lei, F. Castles, C. J. Stevens, C. R. M. Grovenor, and P. S. Grant, "3D printed anisotropic dielectric composite with meta-material features," Mater. Des., vol. 93, pp. 423-430, Mar. 2016.

[303] K. Momeni, S. M. M. Mofidian, and H. Bardaweel, "Systematic design of highstrength multicomponent metamaterials," Mater. Des., vol. 183, p. 108124, Dec. 2019.

[304] R. Gheisari et al., "Multi-material additive manufacturing of low sintering temperature Bi 2 Mo 2 O 9 ceramics with Ag floating electrodes by selective laser burnout," Virtual Phys. Prototyp., vol. 15, no. 2, pp. 133-147, Apr. 2020.

[305] Q. Wang, J. A. Jackson, Q. Ge, J. B. Hopkins, C. M. Spadaccini, and N. X. Fang, "Lightweight Mechanical Metamaterials with Tunable Negative Thermal Expansion," Phys. Rev. Lett., vol. 117, no. 17, p. 175901, Oct. 2016. 
[306] D. Chen and X. Zheng, "Multi-material Additive Manufacturing of Metamaterials with Giant, Tailorable Negative Poisson's Ratios," Sci. Rep., vol. 8, no. 1, pp. 1-8, Dec. 2018.

[307] M. Askari, C. J. Tuck, Q. Hu, R. J. M. Hague, and R. D. Wildman, "Multimaterial Manufacture Through Combining Optical Tweezers with Multiphoton Fabrication," J. Laser Micro/Nanoengineering, vol. 14, no. 1, pp. 80-87, Apr. 2019.

[308] A. I. Kuznetsov et al., "Laser-induced transfer of metallic nanodroplets for plasmonics and metamaterial applications," J. Opt. Soc. Am. B, vol. 26, no. 12, p. B130, Dec. 2009. 